\title{
ATMOSPHERIC PRESSURE
}

CHEMICAL IONIZATION SOURCES

USED IN THE DETECTION OF

EXPLOSIVES BY ION MOBILITY

SPECTROMETRY

\author{
M. J. Waltman
}

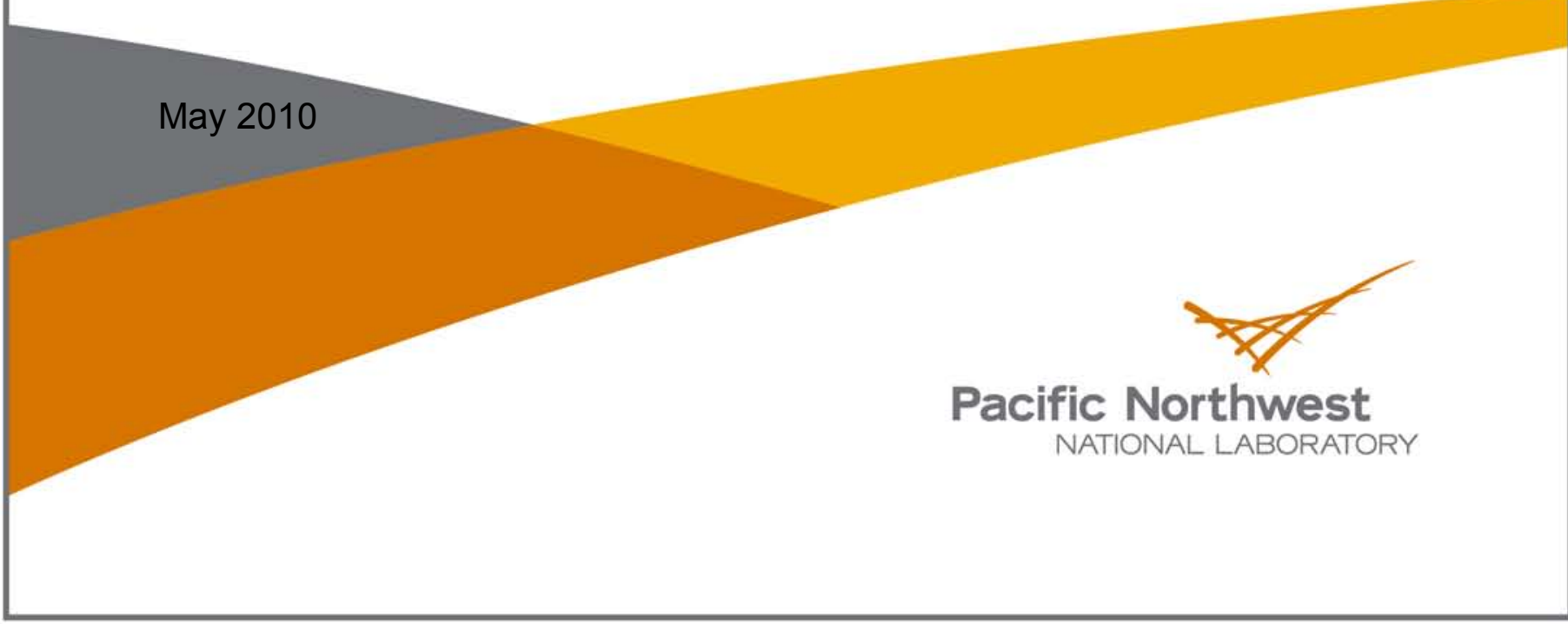




\title{
DISCLAIMER
}

This report was prepared as an account of work sponsored by an agency of the United States Government. Neither the United States Government nor any agency thereof, nor Battelle Memorial Institute, nor any of their employees, makes any warranty, express or implied, or assumes any legal liability or responsibility for the accuracy, completeness, or usefulness of any information, apparatus, product, or process disclosed, or represents that its use would not infringe privately owned rights. Reference herein to any specific commercial product, process, or service by trade name, trademark, manufacturer, or otherwise does not necessarily constitute or imply its endorsement, recommendation, or favoring by the United States Government or any agency thereof, or Battelle Memorial Institute. The views and opinions of authors expressed herein do not necessarily state or reflect those of the United States Government or any agency thereof.

\author{
PACIFIC NORTHWEST NATIONAL LABORATORY \\ operated by \\ BATTELLE \\ for the \\ UNITED STATES DEPARTMENT OF ENERGY \\ under Contract DE-AC05-76RL01830
}

Printed in the United States of America
Available to DOE and DOE contractors from the Office of Scientific and Technical Information,
P.O. Box 62, Oak Ridge, TN 37831-0062;
ph: (865) 576-8401
fax: $(865)$ 576-5728
email: reports@adonis.osti.gov

\begin{abstract}
Available to the public from the National Technical Information Service, U.S. Department of Commerce, 5285 Port Royal Rd., Springfield, VA 22161 ph: (800) 553-6847 fax: $(703) 605-6900$ email: orders@ntis.fedworld.gov online ordering: http://www.ntis.gov/ordering.htm
\end{abstract}

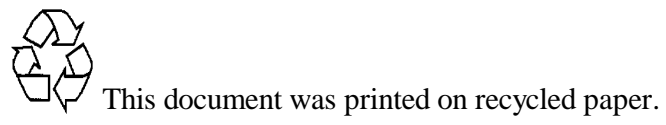




\title{
ATMOSPHERIC PRESSURE CHEMICAL IONIZATION SOURCES USED IN THE DETECTION OF EXPLOSIVES BY ION MOBILITY SPECTROMETRY
}

\author{
by \\ Melanie Jean Waltman \\ Submitted in partial fulfillment of the requirements for the degree of \\ Doctor of Philosophy in Chemistry \\ New Mexico Tech \\ Department of Chemistry \\ Socorro, NM
}

May 2010 


\begin{abstract}
Explosives detection is a necessary and wide spread field of research. From large shipping containers to airline luggage, numerous items are tested for explosives every day. In the area of trace explosives detection, ion mobility spectrometry (IMS) is the technique employed most often because it is a quick, simple, and accurate way to test many items in a short amount of time. Detection by IMS is based on the difference in drift times of product ions through the drift region of an IMS instrument. The product ions are created when the explosive compounds, introduced to the instrument, are chemically ionized through interactions with the reactant ions. The identity of the reactant ions determines the outcomes of the ionization process. This research investigated the reactant ions created by various ionization sources and looked into ways to manipulate the chemistry occurring in the sources.
\end{abstract}

The ionization source most utilized in IMS instruments is ${ }^{63} \mathrm{Ni}$. It is a very reliable and well understood source, but due to safety and regulatory concerns non-radioactive sources are being put to use. One non-radioactive source already implemented into IMS instruments is corona discharge. The predominant reactant ion observed in a point-toplane corona discharge occurs at $\mathrm{m} / \mathrm{z} 60$ in clean air. There have been multiple references in the literature to the identity of this ion with some disagreement. It was postulated to be either $\mathrm{CO}_{3}{ }^{-}$or $\mathrm{N}_{2} \mathrm{O}_{2}{ }^{-}$. The identity of this ion is important as it is a key to the ionization of analytes. The ion at $\mathrm{m} / \mathrm{z} 60$ was determined here to be $\mathrm{CO}_{3}{ }^{-}$through the use of ${ }^{18} \mathrm{O}$ labeled oxygen. Further confirmation was provided through MS/MS studies. An example of the importance of knowing the reactant ion identity was the ionization of nitroglycerine (NG) 
with $\mathrm{CO}_{3}{ }^{-}$which produced the adduct $\mathrm{NG} \cdot \mathrm{CO}_{3}{ }^{-}$. This adduct formation was similar to the ionization of $\mathrm{NG}$ with $\mathrm{NO}_{3}{ }^{-}$and $\mathrm{Cl}^{-}$reactant ions that also formed adducts with $\mathrm{NG}$. The fragmentation patterns of these adducts provides insight into the charge distribution. The fragmentation of the $\mathrm{NG} \cdot \mathrm{NO}_{3}{ }^{-}$adduct produced the nitrate ion whereas fragmentation of the $\mathrm{NG} \cdot \mathrm{Cl}^{-}$adduct also produced the nitrate ion indicating that the charge resided predominantly with the nitrate in both complexes. However, the fragmentation of $\mathrm{NG} \cdot \mathrm{CO}_{3}{ }^{-}$yielded $\mathrm{CO}_{3}{ }^{-}$. This indicates that $\mathrm{CO}_{3}{ }^{-}$has a relatively high electron affinity, higher than that of chlorine and likely close to that of the nitrate ion.

As part of this research a new atmospheric pressure ionization (API) source was developed, characterized and compared to commonly used API sources with both mass spectrometry and ion mobility spectrometry. The source, a distributed plasma ionization source (DPIS), consisted of two electrodes of different sizes separated by a thin glass slide. Application of a high RF voltage across the electrodes generated plasma in air yielding both positive and negative ions. The positive ions generated were similar to those created in a conventional point-to-plane corona discharge ion source, being mass identified as solvated protons of general formula $\left(\mathrm{H}_{2} \mathrm{O}\right)_{n} \mathrm{H}^{+}$, with $\left(\mathrm{H}_{2} \mathrm{O}\right)_{2} \mathrm{H}^{+}$as the most abundant reactant ion. The negative reactant ions produced were mass identified primarily as $\mathrm{CO}_{3}{ }^{-}, \mathrm{NO}_{3}{ }^{-}, \mathrm{NO}_{2}{ }^{-}, \mathrm{O}_{3}{ }^{-}$and $\mathrm{O}_{2}{ }^{-}$of various relative intensities. The predominant ion and relative ion ratios varied depending upon source construction and supporting gas flow rates. A few compounds including drugs, explosives and amines were selected to evaluate the new ionization source. A lifetime experiment was run to test the stability of the source. The source was operated continuously for three months and 
although surface deterioration was observed visually, the source continued to produce ions at a rate similar that of the initial conditions.

The ions created in a discharge were dependent upon experimental conditions. It was postulated that the change in ions was caused by reactions with neutral species $\mathrm{O}_{3}$ and $\mathrm{NO}_{2}$. In an effort to better understand the formation of negative reactant ions in air produced by an atmospheric pressure corona discharge source, the neutral vapors generated by a corona discharge were introduced in varying amounts into the ionization region of an ion mobility spectrometer/mass spectrometer containing a ${ }^{63} \mathrm{Ni}$ ionization source. With no discharge gas, the predominant ions were $\mathrm{O}_{2}{ }^{-}$, however, upon the introduction of low levels of discharge gas the $\mathrm{NO}_{2}{ }^{-}$ion quickly became the dominant species. As the amount of discharge gas increased, the appearance of $\mathrm{CO}_{3}{ }^{-}$was observed followed by the appearance of $\mathrm{NO}_{3}{ }^{-}$. At very high discharge gas levels, $\mathrm{NO}_{3}{ }^{-}$species became effectively the only ion present and appeared as two peaks in the IMS spectrum, $\mathrm{NO}_{3}{ }^{-}$and the $\mathrm{NO}_{3}{ }^{-} \cdot \mathrm{HNO}_{3}$ adduct, with separate mobilities. RDX was examined in order to investigate the ionization properties with these three primary ions. It was found that RDX forms a strong adduct with both $\mathrm{NO}_{2}{ }^{-}$and $\mathrm{NO}_{3}{ }^{-}$with reduced mobility values of 1.49 and $1.44 \mathrm{~cm}^{2} \mathrm{~V}^{-1} \mathrm{~s}^{-1}$, respectively. No adduct was observed for RDX with $\mathrm{CO}_{3}^{-}$ although this adduct has been observed with a corona discharge mass spectrometer. It is believed that this adduct, although formed, does not have a sufficiently long lifetime (greater than $10 \mathrm{~ms}$ ) to be observed in an ion mobility spectrometer.

Many explosives form product ions via adduct formation. Thus, ion identities and subsequent mobility values will change based upon reactant ion identity. Thermal stability of the product ion, sensitivity and selectivity can be altered by the selection of 
the reactant ion. This study investigated the use of $\mathrm{O}_{2}{ }^{-}, \mathrm{Cl}^{-}, \mathrm{NO}_{2}{ }^{-}$, and $\mathrm{NO}_{3}{ }^{-}$reactant ions for explosive ionization. It was shown that NG, PETN, RDX and tetryl form adducts with chloride and nitrate reactant ions. $\mathrm{RDX}$ forms nitrite adducts with $\mathrm{NO}_{2}{ }^{-}$reactant ions and in the presence of $\mathrm{O}_{2}^{-} \mathrm{RDX}$ also forms the nitrite adduct via a 2-step process by first forming the nitrite ion. When $\mathrm{O}_{2}^{-}$or $\mathrm{NO}_{2}{ }^{-}$is the reactant ion $\mathrm{NG}$ forms the nitrate ion and subsequently produces the NG-nitrate adduct. Tetryl fragments to form (tetryl- $\left.\mathrm{NO}_{2}\right)^{-}$with $\mathrm{O}_{2}{ }^{-}$and $\mathrm{NO}_{2}{ }^{-}$reactant ions. TNT is ionized by proton abstraction with $\mathrm{O}_{2}{ }^{-}, \mathrm{Cl}^{-}$, and $\mathrm{NO}_{2}{ }^{-}$ reactant ions. However, when $\mathrm{NO}_{3}{ }^{-}$ions are present TNT is not ionized. Because of the high electron affinity of the nitrate ion, many compounds will not ionize in its presence. The use of nitrate reactant ions result in a reduction of ions created from background interferents while allowing the ionization of selected explosive compounds, making the nitrate ion a good candidate as a selective reactant ion species. The reduced mobility values from mass identified ion mobility peaks are provided for a variety of product ions observed resulting from combinations of the explosives and the reactant ions investigated.

Key words: atmospheric pressure chemical ionization (APCI), ion mobility spectrometry (IMS), mass spectrometry, $\mathrm{CO}_{3}{ }^{-}$, corona discharge, distributed plasma ionization source (DPIS), explosives detection 


\section{ACKNOWLEDGEMENTS}

I would like to thank my advisor, Dr. Robert Ewing, for his guidance and allowing me work in two of his labs. I am appreciative to Dr. Michael Heagy who took care of some things for me when I was not in New Mexico. Thank you to the rest of my committee, Dr. Larry Werbelow, Dr. Michael Pullin, and Dr. Navid Mojtabai, for their time. I am very grateful to Debbie Wallace, who helped with all the paperwork and answered lots of questions.

Thank you to Dr. Herb Hill, Prahba Dwivedi, and Christina Crawford for their collaboration in this research and allowing us to visit their lab and use their instruments. Thank you to my husband, Douglas, my family, and my friends for their years of support, and thanks to God for getting me through this. 


\section{TABLE OF CONTENTS}

Page

List of tables

iv

List of figures

$\mathrm{V}$

List of abbreviations

$\mathrm{X}$

Chapter 1 - Introduction 1

Chapter 2 - Production and Utilization of $\mathrm{CO}_{3}{ }^{-}$Produced by a Corona Discharge for Atmospheric Chemical Ionization

Chapter 3 -Characterization of a Distributed Plasma Ionization Source (DPIS) for Ion Mobility Spectrometry and Mass Spectrometry

Chapter 4 - Mechanisms for Negative Reactant Ion Formation of Air in an Atmospheric Pressure Corona Discharge

Chapter 5-Atmospheric Pressure Chemical Ionization of Explosives

Chapter 6-Conclusions

References 


\section{LIST OF TABLES}

Page

Table 1.1: Electron affinities of typical reactant ions observed at API conditions

Ranges collected from the NIST web page from multiple sources.

Table 4.1: Negative ion reaction rate constants

Table 4.2: Reaction times for reactions 1-7 with varying concentrations of $\left[\mathrm{NO}_{2}\right]$

$=\left[\mathrm{O}_{3}\right]$ as indicated

Table 6.1: $\mathrm{K}_{\mathrm{o}} \mathrm{s}$ and mass values for explosive product ions seen in IMS spectra.

Data in brackets is from WSU, all other from PNNL. 


\section{LIST OF FIGURES}

Page

Figure 1.1: Schematic of an ion mobility spectrometer 2

Figure 1.2: Example spectrum from an IMS $\quad 4$

Figure 1.3: Drawings of explosives with common name and extended name 9

Figure 2.1: Mass spectra of background ions generated (A) in air at 15 atmospheric pressure with a ${ }^{63} \mathrm{Ni}$ ionization source and (B) from a corona discharge ionization in synthetic air at atmospheric pressure

Figure 2.2 MS/MS of collision induced dissociation of $\mathrm{m} / \mathrm{z} 60$ showing only fragment ion of $16\left(\mathrm{O}^{-}\right)$

Figure 2.3: Mass spectra of the corona discharge with $\mathrm{N}_{2}$ and $5 \mathrm{~mL}$ of oxygen

(A) with regular oxygen and (B) with ${ }^{18} \mathrm{O}$ labeled oxygen

Figure 2.4: Mass spectrum of ${ }^{63} \mathrm{Ni}$ with ${ }^{18} \mathrm{O}_{2}$

Figure 2.5: MS/MS of the $\mathrm{m} / \mathrm{z} 62$ ion from the ${ }^{18} \mathrm{O}$ labeled oxygen 21

Figure 2.6: Mass spectra of nitroglycerine (A) from a DPIS ionization source,

(B) from a corona discharge ionization source and (C) from a corona discharge with carbon tetrachloride

Figure 2.7: MS/MS of nitroglycerine (A) from a DPIS ionization source $(\mathrm{m} / \mathrm{z}$ 289, $\left.\mathrm{NG} \cdot \mathrm{NO}_{3}\right),(\mathrm{B})$ from a corona discharge ionization source $(\mathrm{m} / \mathrm{z} 287$, $\mathrm{NG} \cdot \mathrm{CO}_{3}$ ) and $(\mathrm{C})$ from a corona discharge with carbon tetrachloride $\left.m / z 262, \mathrm{NG} \cdot \mathrm{Cl}^{-}\right)$

Figure 3.1: The distributed plasma ion source, DPIS consisting of a 19x19 mm microscope slide cover with $1.2 \mu \mathrm{m}$ thick titanium coating completely 
covering the bottom side (large electrode) and $4 \mathrm{~mm}$ disc on top (small electrode). (A) is the DPIS shown in an open configuration wires were connected to each electrode (B) is the enclosed DPIS contained in a Teflon cylinder.

Figure 3.2: A side view of the electric field gradient simulation of the DPIS with the large electrode on the left side and the small electrode on the right. Lines show the equipotential voltage contours produced by the source.

Figure 3.3: Magnified images over 33 days of the continuous operation of the DPIS. Photograph of the small electrode (A) prior to use, (B) after 6 days, (C) after 16 days and (D) after 33 days.

Figure 3.4: Ion mobility spectra of positive background ions produced by (A) corona discharge and (B) open DPIS from ionization of ambient air.

Figure 3.5: Ion mobility spectra of 2, 4-lutidine produced by (A) corona discharge and (B) open DPIS. IMS peaks of the protonated water reactant ions and protonated monomer ions of the 2, 4-lutidine are shown at mobility values of 2.70 and $1.95 \mathrm{~cm}^{2} \mathrm{~V}^{-1} \mathrm{~s}^{-1}$, respectively.

Figure 3.6: Single ion monitored (SIM) ion mobility spectra of methamphetamine $(\mathrm{m} / \mathrm{z} 150)$, caffeine $(\mathrm{m} / \mathrm{z} 195)$, and 2 , 4-lutidine $(\mathrm{m} / \mathrm{z}$ 108) using the open DPIS.

Figure 3.7: Mass spectra of negative ions generated in purified air with ${ }^{63} \mathrm{Ni}$, point-to-plane corona, open DPIS, and enclosed DPIS.

Figure 3.8: IMS spectra of negative ions produced by the atmospheric pressure 
ionization of clean air at ambient temperature with ${ }^{63} \mathrm{Ni}$ and an enclosed DPIS.

Figure 3.9: Mass spectra of RDX $(\mathrm{mw}=222)$. Ionization of $\mathrm{RDX}$ with the $(\mathrm{A})$ enclosed DPIS and (B) point-to-plane corona discharge.

Figure 4.1: A diagram of the Nalgene bottle used for the Static and Dynamic Container experiments. The Static Container was prepared by first by flushing the container with the desired gas, then stopping the flow and withdrawing an aliquot with a syringe. The Dynamic Container used a continuous flow of gas and the outlet was interfaced to the IMS.

Figure 4.2: A diagram of the Continuous Flow Tube apparatus consisting of a 1/4" stainless steel Swagelok cross. The discharge occurs at the center of this cross where two 0.026 " diameter metal wires meet separated by a glass slide. A continuous stream of purified air passes over the discharge and a fraction of the effluent is presented to the inlet of the IMS

Figure 4.3: IMS-F spectra of (a) zero air, (b) $2 \mathrm{~mL}$ of gas sampled from the Static Container with zero air after 1 minute discharge and (c) $2 \mathrm{~mL}$ of gas sampled from the Static Container with $\mathrm{O}_{2}$ after 1 minute discharge

Figure 4.4: (a) ion mobility spectrum and (b) mass spectrum of $2 \mathrm{~mL}$ of gas sampled from the Static Container with zero air after the discharge was operated for 5 minutes

Figure 4.5: IMS spectra of discharge gas from the Continuous Flow Tube collected with the electron multiplier in the mass spectrometer. The 
IMS-EM, displayed on top, shows the total ion current followed by selected ion monitoring of $m / z$ 32, 46, 60 and 62 in order from top to bottom

Figure 4.6: IMS peak intensities at increasing discharge times. The source was operated at a $50 \%$ duty cycle with $40 \mathrm{~mL} / \mathrm{min}$ continuous flow of zero air through the Dynamic Container and into the IMS

Figure 4.7: IMS-F spectra of discharge gas from the Continuous Flow Tube with (a) no discharge, (b) low flow, (c) medium flow and (d) high flow

Figure 4.8: IMS-F spectra of RDX with different ion chemistries generated from varying levels of discharge gas from the Continuous Flow Tube (a) low flow, (b) medium flow and (c) high flow

Figure 5.1: IMS Spectra (PCP) of reactant ion peaks generated from a ${ }^{63} \mathrm{Ni}$ ionization source at $110^{\circ} \mathrm{C}$. A) Air showing only $\mathrm{O}_{2}{ }^{-} \mathrm{B}$ ) addition of carbon tetrachloride producing $\mathrm{Cl}^{-}, \mathrm{C}$ ) low concentrations of neutral vapors (ozone and $\mathrm{NO}_{\mathrm{x}}$ ) generated in a DPIS producing $\mathrm{NO}_{2}^{-}$and $\mathrm{D}$ ) higher concentrations of neutral vapors (ozone and $\mathrm{NO}_{\mathrm{x}}$ ) generated in a DPIS ionization source producing $\mathrm{NO}_{3}{ }^{-}$and $\mathrm{HNO}_{3} \cdot \mathrm{NO}_{3}{ }^{-}$ions.

Figure 5.2: Mass spectra (API-III) of clean dry air at ambient temperatures A) using the enclosed DPIS ionization source producing $\mathrm{NO}_{3}{ }^{-}$at $\mathrm{m} / \mathrm{z} 62$, B) using point-to-plane corona discharge source producing $\mathrm{CO}_{3}{ }^{-}$at $\mathrm{m} / \mathrm{z}$ 60 , C) using point to plane corona discharge with the addition of carbon tetrachloride producing predominantly $\mathrm{Cl}^{-}$at $\mathrm{m} / \mathrm{z} 35$ and 37 .

Figure 5.3: IMS spectra (PCP) of $10 \mathrm{ng}$ RDX with a variety of reactant ion 
species as shown in Figure 5.1 A) $\left.\left.\mathrm{O}_{2}^{-}, \mathrm{B}\right) \mathrm{Cl}^{-}, \mathrm{C}\right) \mathrm{NO}_{2}{ }^{-}$and D) $\mathrm{NO}_{3}{ }^{-}$ and $\mathrm{HNO}_{3} \cdot \mathrm{NO}_{3}{ }^{-}$.

Figure 5.4: IMS spectra with the same data as in Figure 5.3 with the $\mathrm{x}$-axis plotted as $1 / \mathrm{K}_{0}$.

Figure 5.5: Mass spectra (API-III) of RDX at atmospheric pressure with a variety of reactant ion species as shown in Figure 5.2 with RIPs of A) $\mathrm{NO}_{3}{ }^{-}$, B) $\mathrm{CO}_{3}{ }^{-}$, and C) $\mathrm{Cl}^{-}$.

Figure 5.6: Three dimensional IMS and MS spectra (WSU) of PETN with DPIS ionization source at $110^{\circ} \mathrm{C}$ showing $\mathrm{NO}_{3}{ }^{-}$as reactant ion and the PETN $\cdot \mathrm{NO}_{3}{ }^{-}$adduct at $\mathrm{m} / \mathrm{z} 378$ and approximately $18.5 \mathrm{~ms}$.

Figure 5.7: IMS/MS spectra (PCP) of tetryl at $110{ }^{\circ} \mathrm{C}$ with $\mathrm{O}_{2}^{-}$: an IMS spectra showing reactant ion peak, an IMS-EM, and a IMS-SIM of m/z 241 . 


\section{LIST OF ABBREVIATIONS}

\begin{tabular}{|c|c|}
\hline A & ampere \\
\hline APCI & atmospheric pressure chemical ionization \\
\hline amu & atomic mass unit \\
\hline CID & collisionally induced dissociation \\
\hline DPIS & distributed plasma ionization source \\
\hline $\mathrm{eV}$ & electron volt \\
\hline IMS & ion mobility spectrometry \\
\hline IMS-EM & IMS spectra with electron multiplier detector \\
\hline IMS-F & IMS spectra with faraday plate detector \\
\hline IMS-SIM & IMS with single ion monitoring \\
\hline MS & mass spectrometry \\
\hline$m / z$ & mass-to-charge ratio \\
\hline NG & nitroglycerin \\
\hline NIST & National Institute of Science and Technology \\
\hline PETN & pentaerythritol tetranitrate \\
\hline $\mathrm{RF}$ & radio frequency \\
\hline RIP & reactant ion peak \\
\hline RDX & 1,3,5-trinitro-1,3,5-triazacyclohexane \\
\hline SIM & single ion monitoring \\
\hline Tetryl & 2,4,6-trinitrophenyl-N-methylnitramine \\
\hline TNT & trinitrotoluene \\
\hline
\end{tabular}




$\begin{array}{ll}\text { TOF-MS } & \text { time-of-flight mass spectrometry } \\ \text { UHP } & \text { ultra high purity } \\ \text { V } & \text { volts } \\ \text { VDC } & \text { volts direct current } \\ \text { WSU } & \text { Washington State University }\end{array}$




\section{CHAPTER 1 \\ INTRODUCTION}

The detection of explosives is a matter of great concern to both the military and civilian sectors. Applications include safeguarding travel, locating hidden mines, scanning mail, and monitoring contaminated sites [1]. Many analytical techniques including liquid chromatography, gas chromatography, chemiluminescence, ion mobility spectrometry, mass spectrometry, and various spectroscopic methods have been used in trace explosives detection [2]. Each method has its own advantages and drawbacks, yet ion mobility spectrometry (IMS) stands apart from the rest as a field deployable technique. A significant reason why IMS is readily used in the field is that it operates at atmospheric pressure and thus does not require vacuum pumps like mass spectrometry does. Since air can be used as the supporting gas, gas cylinders are not required. Because of these factors IMS is a lightweight, portable, field deployable instrument. It is also a relatively low cost instrument that offers real time analysis; a single run on an IMS takes only milliseconds with a normal scan requiring less than 10 seconds. Due to its ease of use, reliability, and ruggedness, IMS is the most widely used technique for trace detection of explosives for both airports and the military [3].

\section{Ion Mobility Spectrometry}

Ion mobility spectrometry works on the basis that the ions of different chemicals will have different mobilities allowing for detection and identification. The mobility of an ion is determined by its drift velocity (dependent upon its size and charge) in a weak 
electric field at atmospheric pressure. This difference in mobility between compounds allows for their separation, and thus detection. Figure 1.1 is a diagram of an ion mobility spectrometer.

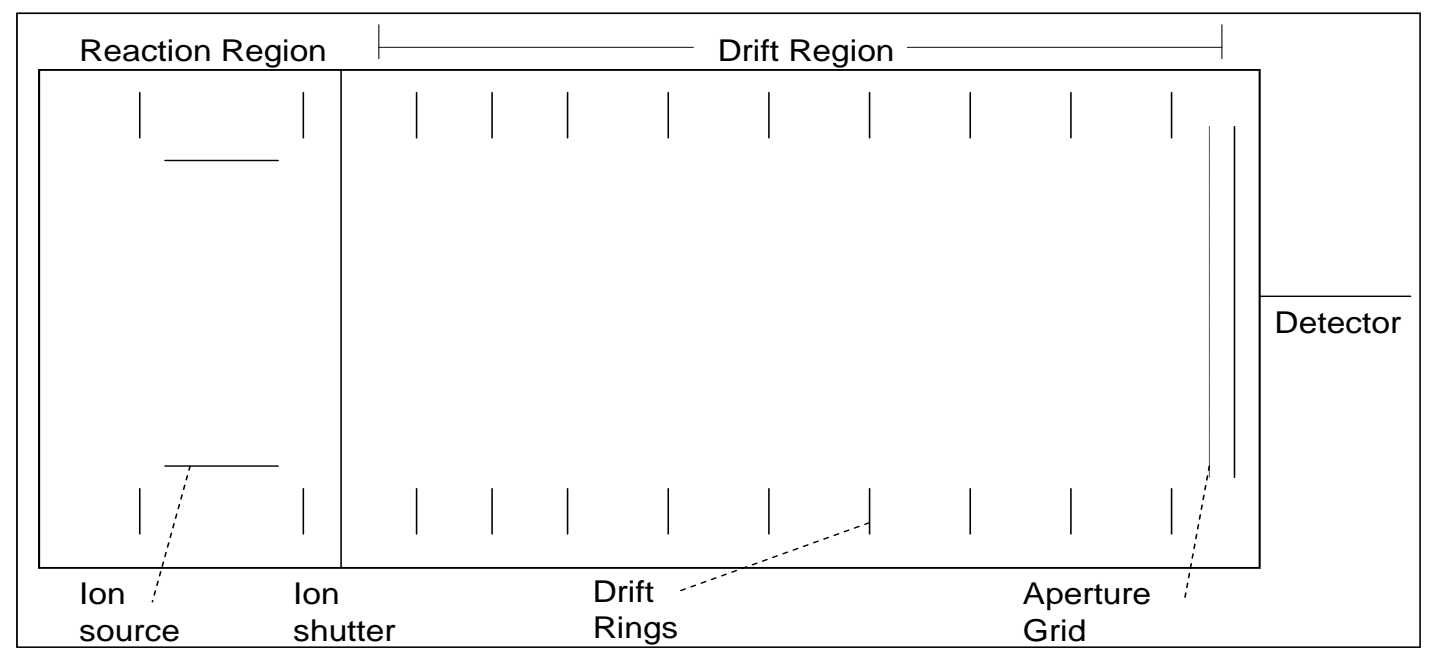

Figure 1.1: Schematic of an ion mobility spectrometer.

First, the sample is ionized in the reaction region. When the ion shutter opens it allows a narrow packet of ions into the drift region. The packet is separated into groups of similar ions that drift at a constant velocity through the electric field against the flow of the drift gas. The speed of ion movement is much faster than the speed of the gas flow. The ions separate based upon size, with smaller ions traveling faster than larger ions. The drift gas keeps the drift region free of neutral analyte molecules and thus prevents reactions from occurring in the drift region causing distortions in the spectra. The drift velocity of an ion is given by [4]:

$$
\mathrm{v}_{\mathrm{d}}=\mathrm{K} \mathrm{E}
$$

where $\mathrm{K}$ is the mobility of the ion packets and $\mathrm{E}$ is the applied electric field. Typical drift velocities for ions of low mass (14 to 500amu) in weak electric fields $(150-300 \mathrm{~V} / \mathrm{cm})$ are 
$1-10 \mathrm{~m} / \mathrm{s}$ at ambient pressure with temperatures from $25-250^{\circ} \mathrm{C}$ [5]. The mobility of an ion packet is given by:

$$
\mathrm{K}=(3 / 16)\left[\left(1.602 \times 10^{-19} \mathrm{z} / \mathrm{N}_{\mathrm{o}}\right)(2 \pi / \mu \mathrm{kT})^{1 / 2}\left(1 / \Omega_{\mathrm{D}}\right)\right] \text {, }
$$

where $\mathrm{z}$ is the charge of the ion, $\mathrm{N}_{\mathrm{o}}$ is the number density of the drift gas, $\mu$ is the reduced mass of the ion-drift gas pair, $\mathrm{k}$ is the Boltzmann's constant, $\mathrm{T}$ is the operating temperature (in Kelvin), and $\Omega_{\mathrm{D}}$ is the ion-neutral average cross section. As can be seen from the equation, mobility is affected not only by the mass of ions, but by their shape and size. Because of this, ions with the same mass but different functional groups can have different mobilities.

After the ion packets make it through the drift region, they go through the aperture grid to the faraday plate detector. As the packets hit the detector a current flow is created and amplified then converted to voltage and reported as the instrument response.

An example of a typical IMS spectrum is shown in Figure 1.2. Peak A is the reactant ion peak (RIP), peak D is the product ion peak, and peaks B and C are fragments of the product ion. Mobilities for the peaks would be calculated from the drift time based on instrument parameters. The mobilities are normalized to 760 Torr and $273 \mathrm{~K}$ to give the reduced mobility, $\mathrm{K}_{\mathrm{o}}$ :

$$
\mathrm{K}_{\mathrm{o}}=\mathrm{K}(\mathrm{P} / 760)(273 / \mathrm{T})[4] .
$$

Reduced mobility values are independent of instrumental or environmental parameters and are specific for an analyte ion. The reduced mobility values can be used for compound identification from different instruments or different environmental conditions. 


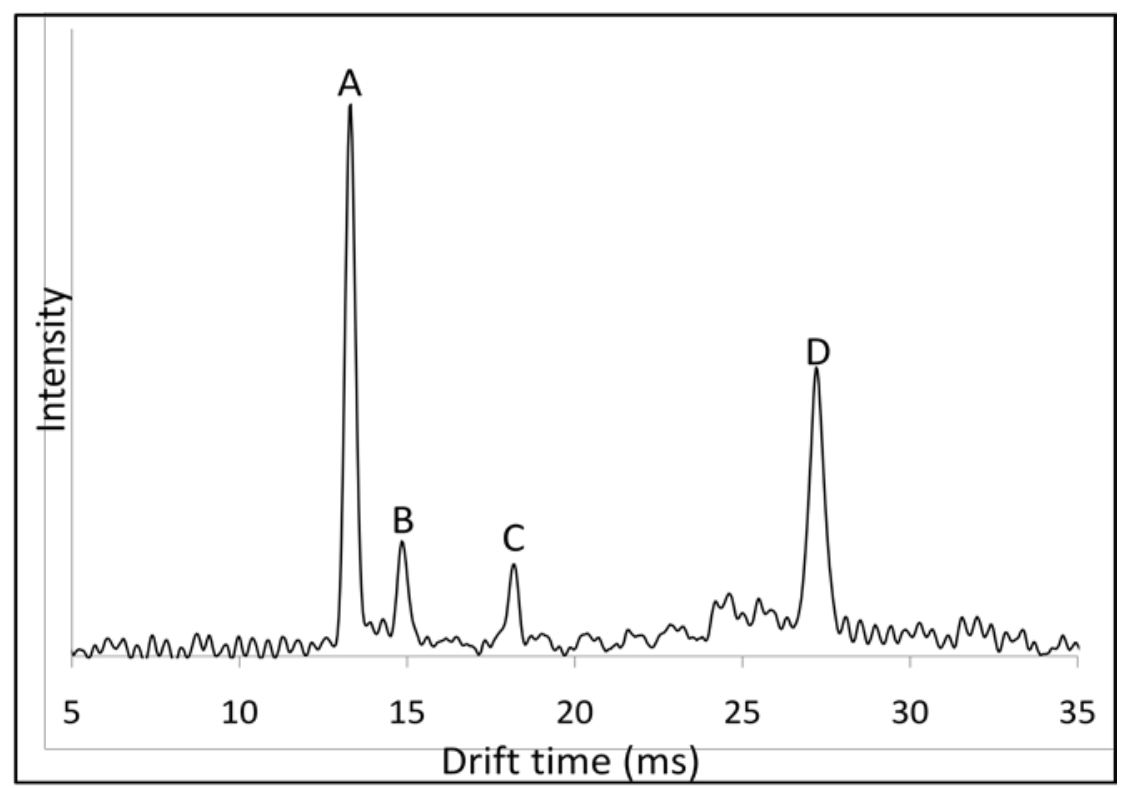

Figure 1.2: Example spectrum from an IMS.

Resolution in IMS is similar to that of HPLC. In chromatography terms, peak capacity of IMS is $80-400$, and peak capacity of liquid chromatography is $50-500$. Peak capacity for gas chromatography is higher at 2000-5000 [6]. Although IMS is considered to have low resolution, the selectivity of IMS cannot be compared by peak capacity alone. In IMS, the chemical ionization process which occurs at atmospheric pressure provides another level of selectivity where analyte ionization is determined by the reactant ion species. Even though IMS is considered to have lower resolution than mass spectrometry, there are some instances where IMS can separate species where mass spectrometry cannot. Examples have been shown in the separation of isomers by IMS [7]. This is possible since separation in IMS is based on ion size and not necessarily mass.

\section{Ionization sources}

The source most commonly used in IMS, due to its reliability, efficiency, and long lifetime, is ${ }^{63} \mathrm{Ni}$, which is a beta (electron) emitter. When the electrons collide with 
air molecules at ambient pressure, ions are formed along with more free electrons of lower energy. This continues until the electrons are at near thermal energies.

$$
\mathrm{N}_{2}+\mathrm{e}^{-} \rightarrow \mathrm{N}_{2}^{+}+2 \mathrm{e}^{-}
$$

In positive polarity, ions are formed through a series of reactions shown below that result in the formation of protonated water clusters $\mathrm{H}^{+}\left(\mathrm{H}_{2} \mathrm{O}\right)_{n}$ [8].

$$
\begin{aligned}
& \mathrm{N}_{2}^{+}+2 \mathrm{~N}_{2} \rightarrow \mathrm{N}_{4}^{+}+\mathrm{N}_{2} \\
& \mathrm{~N}_{4}^{+}+\mathrm{H}_{2} \mathrm{O} \rightarrow \mathrm{H}_{2} \mathrm{O}^{+}+2 \mathrm{~N}_{2} \\
& \mathrm{H}_{2} \mathrm{O}^{+}+\mathrm{H}_{2} \mathrm{O} \rightarrow \mathrm{H}_{3} \mathrm{O}^{+}+\mathrm{OH} \\
& \mathrm{H}_{3} \mathrm{O}^{+}+\mathrm{H}_{2} \mathrm{O}+\mathrm{N}_{2} \leftrightarrow \mathrm{H}^{+}\left(\mathrm{H}_{2} \mathrm{O}\right)_{2}+\mathrm{N}_{2} \\
& \mathrm{H}^{+}\left(\mathrm{H}_{2} \mathrm{O}\right)_{2}+\mathrm{H}_{2} \mathrm{O}+\mathrm{N}_{2} \leftrightarrow \mathrm{H}^{+}\left(\mathrm{H}_{2} \mathrm{O}\right)_{3}+\mathrm{N}_{2}
\end{aligned}
$$

This reservoir of positive ions contains the reactant ions available for chemical ionization of an analyte. In chemical ionization, some analytes, such as explosives, ionize more efficiently with negative reactant ions. Negative reactant ions are formed when the thermalized electrons are captured by oxygen in three-body collisions:

$$
\begin{aligned}
& \mathrm{O}_{2}+\mathrm{e}^{-}+\mathrm{M} \rightarrow \mathrm{O}_{2}{ }^{-}+\mathrm{M} \\
& \mathrm{O}_{2}{ }^{-}+\mathrm{H}_{2} \mathrm{O}+\mathrm{M} \leftrightarrow \mathrm{O}_{2}{ }^{-}\left(\mathrm{H}_{2} \mathrm{O}\right)+\mathrm{M} \\
& \mathrm{O}_{2}{ }^{-} \mathrm{H}_{2} \mathrm{O}+\mathrm{H}_{2} \mathrm{O}+\mathrm{M} \leftrightarrow \mathrm{O}_{2}{ }^{-}\left(\mathrm{H}_{2} \mathrm{O}\right)_{2}+\mathrm{M}
\end{aligned}
$$

creating a reservoir of $\mathrm{O}_{2}^{-}\left(\mathrm{H}_{2} \mathrm{O}\right)_{n}$ ions.

Although ${ }^{63} \mathrm{Ni}$ is normally used in IMS, there are regulatory and safety issues associated with radioactive sources, and thus alternative ionization sources are desired if they can have equal reliability. Therefore, there have been several investigations into the development of non-radioactive sources for atmospheric pressure chemical ionization (APCI) to be used with ion mobility spectrometry (IMS) and mass spectrometry (MS), 
including electrospray [9], photoionization [10] and corona discharge [11]. Electrospray ionization requires the use of solvents, pumps, an additional power supply and a heating element for desolvation of ions produced and is predominantly used for liquid and nonvolatile samples [9]. Photoionization requires the use of an extra power supply and a discharge lamp and provides selective ionization. For example alcohols can be ionized with a $10.6 \mathrm{eV}$ discharge lamp [10]. Corona discharge sources require an additional power supply. Of these sources; corona discharge appears to provide the most similar ionization properties to that of ${ }^{63} \mathrm{Ni}$ sources [11].

A primary disadvantage of the corona discharge source for use with IMS is the degradation in performance and eventual failure due to erosion at the discharge point $[12,13]$ (typically the tip of a needle or fine wire). Despite these shortcomings, the desire for a non-radioactive source for IMS has led to the development of corona discharge ionization sources for IMS. For instance, a commercial hand-held IMS with a corona discharge ionization source is currently available from Smiths Detection (Watford, UK) [14]. In corona discharge, the creation of negative reactant ions is different than those created from ${ }^{63} \mathrm{Ni}$ sources. Corona discharge sources typically produce nitrite and nitrate ions with limited reactivity due to their inherently high electron affinities [11]. One of the goals in the development of IMS systems with corona discharge ionization sources was to produce reactant ions similar to those generated with a ${ }^{63} \mathrm{Ni}$ source, mainly $\mathrm{O}_{2}{ }^{-}$ions. To achieve this, a pulsed corona was explored [11] as was adding a counter-current flow to the source region [15]. It is believed that ozone and $\mathrm{NO}_{\mathrm{x}}$ generated in the discharge cause the production of nitrite and nitrate ions. Providing a short pulse or counter-current flow resulted in a decrease in the amount of neutral ozone or $\mathrm{NO}_{x}$, which enabled $\mathrm{O}_{2}^{-}$reactant 
ions to persist in the reaction region. In addition to the nitrite and nitrate ions, corona discharge sources have also been shown to produce $\mathrm{O}_{2}{ }^{-}, \mathrm{O}_{3}{ }^{-}$and an anion at $\mathrm{m} / \mathrm{z} 60$ along with hydrates of these ions $[16,17]$.

Differences in the reactivity that exist between the assortment of ions that can be produced from a variety of ionization sources needs to be understood for the development of reliable instruments. Since the ionization of explosives, pesticides, blister agents and other chemicals rely on negative atmospheric pressure chemical ionization, the identity of the negative reactant ions will affect subsequent product ion formation. Changes to the ionization mechanism of the products such as charge transfer, proton abstraction or adduct formation can lead to changes in sensitivity or ion identity. For example, if the ionization mechanism is charge transfer, relative electron affinities between the reactant ion and the analyte will determine the potential for ionization to occur. The ranges of electron affinities for the various anions discussed are provided in Table 1.1. IMS relies on mobility as the means for identification and changes to ion identity will change the mobility values and subsequent detection algorithms.

\begin{tabular}{|c|c|}
\hline Molecule & Electron affinity \\
\hline $\mathrm{Cl}$ & $3.6 \mathrm{eV}$ \\
\hline $\mathrm{O}_{2}$ & $0.40-1.3 \mathrm{eV}$ \\
\hline $\mathrm{O}_{3}$ & $1.8-2.5 \mathrm{eV}$ \\
\hline $\mathrm{NO}_{2}$ & $1.8-3.9 \mathrm{eV}$ \\
\hline $\mathrm{NO}_{3}$ & $3.7-3.9 \mathrm{eV}$ \\
\hline $\mathrm{CO}_{3}$ & $1.8-3.5 \mathrm{eV}$ \\
\hline
\end{tabular}

Table 1.1: Electron affinities of typical reactant ions observed at API conditions. Ranges collected from the NIST web page from multiple sources. [18] 


\section{Explosives ionization}

The explosive compounds examined in this research are observed predominantly as negative ions due to their high electronegativities. These explosive compounds ionize in many different ways, the four most common are as follows.

$$
\begin{array}{ll}
\text { Proton abstraction } & \mathrm{M}+\mathrm{O}_{2}^{-} \rightarrow(\mathrm{M}-\mathrm{H})^{-}+\mathrm{HO}_{2} \\
\text { Electron transfer } & \mathrm{M}+\mathrm{O}_{2}^{-} \rightarrow \mathrm{M}^{-}+\mathrm{O}_{2} \\
\text { Adduct formation } & \mathrm{M}+\mathrm{O}_{2}^{-} \rightarrow \mathrm{M} \cdot \mathrm{O}_{2}^{-} \\
\text {Fragmentation } & \mathrm{M}+\mathrm{O}_{2}^{-} \rightarrow \mathrm{X}^{-}+(\mathrm{M}-\mathrm{X})+\mathrm{O}_{2}
\end{array}
$$

TNT ionizes by electron transfer into $\mathrm{TNT}^{-}$, and by proton abstraction $(\mathrm{TNT}-\mathrm{H})^{-}$and by fragmentation $\left(\mathrm{TNT}-\mathrm{NO}_{2}\right)^{-}[19]$. Explosives like RDX first ionize by the production of $\mathrm{NO}_{2}{ }^{-}$groups which in turn form adducts with neutral RDX molecules to create RDX. $\mathrm{NO}_{2}{ }^{-}$ [20]. In these situation, the nitrite ion and its adduct are observed in the IMS spectra. Some other explosives such as tetryl are not well characterized or discussed in the literature. Figure 1.3 displays drawings and names of some common explosives. 
<smiles>O=[N+]([O-])OCC(CO[N+](=O)[O-])(CO[N+](=O)[O-])CO[N+](=O)[O-]</smiles>

PETN Pentaerythritol tetranitrate<smiles>O=[N+]([O-])N1CN([N+](=O)[O-])CN([N+](=O)[O-])C1</smiles>

RDX

1,3,5-trinitro-1,3,5-triazacyclohexane<smiles>Cc1c([N+](=O)[O-])cc([N+](=O)[O-])cc1[N+](=O)[O-]</smiles>

TNT

2,4,6-trinitrotoluene<smiles>CN(c1c([N+](=O)[O-])cc([N+](=O)[O-])cc1[N+](=O)[O-])[N+](=O)[O-]</smiles>

Tetryl

2,4,6-trinitrophenyl-N-methylnitramine

Figure 1.3: Drawings of explosives with common name and extended name.

\section{Purpose of research}

This dissertation consists of four separate, but related, research experiments (chapters) which were conducted in order to probe the chemistry involved in the ionization of explosives by APCI sources. Chapters 3 and 4 have previously been published, and chapters 2 and 5 are in preparation for publication. They are placed in the order in which the experiments were performed.

In order to properly understand the ionization of explosives in an IMS, the reactant ions formed in the ionization process must be identified. Chapter 2 establishes the identity of an important reactant ion created in a point-to-plane corona discharge source and how it differs from the ions created in a ${ }^{63} \mathrm{Ni}$ source. Along these lines, Chapter 3, Waltman MJ, Dwivedi P, Hill HH, Blanchard WC, Ewing RG, Talanta 2008, 77,249 , describes a distributed plasma ionization source (DPIS) which is a newly 
developed source. Ions created using the DPIS differ from ions formed in both ${ }^{63} \mathrm{Ni}$ and corona discharge sources.

Chapter 4, Ewing RG, Waltman MJ, International Journal for Ion Mobility

Spectrometry 2009, 12, 65, discusses the investigation of ionization kinetics involved in forming the reactant ions in a discharge source. Understanding the paths taken to produce the ions detected in an IMS enables parameters to be adjusted to select specific reactant ions. The use of specific reactant ions can improve the detection of explosives providing better reliability and selectivity.

Chapter 5 examines the negative ionization processes involved in the ionization of explosive compounds with a variety of reactant ion species. An assortment of explosives, some well characterized and some not previously reported in the literature, are investigated with these reactant ions. The ions produced by atmospheric pressure ionization are identified by mass spectrometry. Reduced mobility values for the variety of ions generated were measured and compared to literature values where available. The goal was to obtain an understanding of ion formation processes to determine optimal ionization parameters. Another goal was to find the best reactant ion species available for selective ionization of explosive compounds. This research compares explosives of different functional groups in their response to multiple ionization sources in an IMS instrument. 


\section{CHAPTER 2}

\section{PRODUCTION AND UTILIZATION OF $\mathrm{CO}_{3}{ }^{-}$PRODUCED BY A CORONA DISCHARGE FOR ATMOSPHERIC PRESSURE CHEMICAL IONIZATION}

We have observed in API-MS studies of negative ions generated in air with a point-to-plane corona discharge that the predominant ion observed under normal operating conditions and gas flows is an ion at $\mathrm{m} / \mathrm{z} 60$. A nominal mass of 60 could occur with an ion containing either 1 carbon and 3 oxygen atoms or 2 nitrogen and 2 oxygen atoms. With the limits of resolution in a quadrupole system, it is impossible to tell the difference between $\mathrm{CO}_{3}{ }^{-}$and $\mathrm{N}_{2} \mathrm{O}_{2}{ }^{-}$. There is some uncertainty in the literature as to the identity of the ion at $\mathrm{m} / \mathrm{z} 60$ formed at atmospheric pressure with a corona discharge, being either $\mathrm{CO}_{3}{ }^{-}$, or $\mathrm{N}_{2} \mathrm{O}_{2}{ }^{-}$. Many studies have indicated the formation of $\mathrm{CO}_{3}{ }^{-}$with mechanisms for the formation involving $\mathrm{O}_{3}{ }^{-}$and $\mathrm{CO}_{2}$ although many of these were performed at sub-ambient pressures $(5-27 \mathrm{kPa})[16]$. Other studies have shown the generation of $\mathrm{N}_{2} \mathrm{O}_{2}{ }^{-}$anion by electron beam ionized free jet expansion or pulsed electric discharge of $\mathrm{O}_{2}$ and $\mathrm{N}_{2}$, pure $\mathrm{N}_{2} \mathrm{O}$, and $\mathrm{NO}$ and argon [21,22]. Through pulsed photoelectron spectroscopy three distinguishable forms of $\mathrm{N}_{2} \mathrm{O}_{2}{ }^{-}$were observed including $\mathrm{O}_{2}^{-} \cdot \mathrm{N}_{2}, \mathrm{O}^{-} \cdot \mathrm{N}_{2} \mathrm{O}$, or $\mathrm{NO}^{-} \cdot \mathrm{NO}$. This was noted by Dono et al. while looking at the abatement of VOCs by corona discharge [23]. Dono et al. noted the dilemma in ion identity mentioned above and they favored $(\mathrm{NO})_{2}{ }_{2}^{-}$since $\mathrm{NO}$ is a known product of discharges in air. Further studies of corona discharges have discussed the production $\mathrm{N}_{2} \mathrm{O}$ along with ozone [24] lending to the possibility of the formation of either $\mathrm{CO}_{3}{ }^{-}$or $\mathrm{N}_{2} \mathrm{O}_{2}{ }^{-}$. Siegel noted the presence of the $\mathrm{m} / \mathrm{z} 60$ and recognized that it could have been $\mathrm{CO}_{3}{ }^{-}$but 
believed it was an adduct of $\mathrm{O}_{2}{ }^{-}$with $\mathrm{N}_{2}$ [25]. The goal of this study was to definitively identify $\mathrm{m} / \mathrm{z} 60$ ion created in a corona discharge in air.

\section{Experimental}

Chemicals and Gases

UHP nitrogen, UHP argon and oxygen were obtained from Matheson Trigas, (Albuquerque, NM). Zero air was generated from a pure air generator from Puregas, LLC (Broomfield, Colorado). ${ }^{18} \mathrm{O}$ labeled oxygen, 95\% purity, was obtained from Cambridge Isotope Laboratories, Inc. (Andover, MA). Nitroglycerine (NG) standard was obtained from Cerilliant (Round Rock, TX) at $1000 \mu \mathrm{g} / \mathrm{mL}$ in acetonitrile and working solutions were diluted in methanol to 1:10 for concentrations of $100 \mathrm{ng} / \mu \mathrm{L}$. Carbon tetrachloride was from Fisher Scientific.

\section{Instrumentation}

The atmospheric pressure ionization triple quadrupole mass spectrometer used in these studies was an API-III (Sciex, Rexdale, Ontario). Data was collected on a Macintosh Quadra 400 using API-Tune software provided with the instrument. It was operated with the factory heated nebulizer probe and a corona discharge ionization source. Typical interface voltages (IN) were between -300 and -600 VDC. The negative corona discharge current was set at $3 \mu \mathrm{A}$. The other lens voltages were as follows: Pinhole $(\mathrm{OR})=-35, \mathrm{R} 0=-30, \mathrm{R} 1=-27, \mathrm{R} 2=45, \mathrm{R} 3=70$. Argon was used as the collision gas for collision induced dissociation (CID) studies. Nitrogen was used as the 
auxiliary and curtain gases with flows of $2000 \mathrm{~mL} / \mathrm{min}$ and $400 \mathrm{~mL} / \mathrm{min}$, respectively. Oxygen was added to the nebulizer port at a rate of $5 \mathrm{~mL} / \mathrm{min}$.

To obtain spectra generated with a ${ }^{63} \mathrm{Ni}$ ionization source, the corona needle was removed and a cylindrical ${ }^{63} \mathrm{Ni}$ source housed within a Teflon block was placed at the end of the nebulizer probe. Sample flow from the heated nebulizer passed through the ${ }^{63} \mathrm{Ni}$ source towards the inlet of the mass spectrometer. The source was floated -400 VDC below the interface voltage so that anions would move from the ${ }^{63} \mathrm{Ni}$ source towards the mass spectrometer. For MS/MS operations argon was used as the collision gas with a collision gas thickness of $125 \times 10^{12}$ molecules $\mathrm{cm}^{-2}$. The voltage measured between OR and R2, which determines the energy of the ion entering the collision cell, was $80 \mathrm{~V}$. Aside from the point-to-plane corona and the ${ }^{63} \mathrm{Ni}$ ionization sources, another discharge sources was used to investigate the ionization of NG. This source, a distributed plasma ion source (DPIS), is described in detail elsewhere [26]. It consisted of a 19 $\mathrm{mm} \times 19 \mathrm{~mm}$ microscope slide cover that was $0.018 \mathrm{~mm}$ thick. One side of the slide cover was completely coated with titanium (large electrode), while the other side had a $4 \mathrm{~mm}$ disk of titanium in the center (small electrode). The titanium was sputtered onto the glass cover to an approximate thickness of $1.2 \mu \mathrm{m}$. Wires were attached to each electrode and connected to a lab built power supply that provided an approximately $6 \mathrm{kV}$ peak to peak $\mathrm{RF}$ voltage at a frequency of $100 \mathrm{kHz}$. The glass slide cover was placed between a copper washer and a Teflon cylinder $32 \mathrm{~mm}$ o.d., $13 \mathrm{~mm}$ i.d. and $10 \mathrm{~mm}$ thick. The large electrode on the slide cover was placed on the copper washer so the small electrode was in the center of the Teflon cylinder. The cylinder, source and copper washer were held 
together by four nylon screws. Similar to the ${ }^{63} \mathrm{Ni}$ ionization source, the DPIS was placed on the end of the nebulizer and floated -400 VDC below the interface voltage.

Solutions of $100 \mu \mathrm{L}$ of methanol containing $10 \mathrm{ug}$ of NG were added to the glass liner of the heated nebulizer while at ambient temperature and no gas flow. After the solvent was allowed to evaporate, the nebulizer heater was turned on (set point of $150{ }^{\circ} \mathrm{C}$ ) to desorb the explosive. At this time the auxiliary and nebulizer flows were turned on at rates of $500 \mathrm{ml} / \mathrm{min}$ and $100 \mathrm{ml} / \mathrm{min}$, respectively. Significant quantities of NG remained in the ionization region and were persistent in the spectra for approximately 10-20 minutes. Each spectrum collected was the result of approximately 25 scans (averaged).

\section{Results and Discussion}

Representative mass spectra of ions generated at atmospheric pressure of dry air are displayed in Figure 2.1. Figure 2.1A represents ions formed with a ${ }^{63} \mathrm{Ni}$ source and Figure 2.1B ions formed with a point-to-plane corona discharge ionization source.

Predominant ions generated with ${ }^{63} \mathrm{Ni}$ include $\mathrm{O}_{2}{ }^{-}, \mathrm{O}_{2}{ }^{-} \cdot \mathrm{H}_{2} \mathrm{O}$ and $\mathrm{O}_{2}{ }^{-} \cdot \mathrm{CO}_{2}$ at $\mathrm{m} / \mathrm{z}$ of 32,50 and 76 amu, respectively. Typical ions generated with the point-to-plane corona discharge are shown at $\mathrm{m} / \mathrm{z} 32,48$ and 60 which are $\mathrm{O}_{2}{ }^{-}, \mathrm{O}_{3}{ }^{-}$and the ion presumed to be either $\mathrm{CO}_{3}{ }^{-}$or $\mathrm{N}_{2} \mathrm{O}_{2}{ }^{-}$. The ion observed at $\mathrm{m} / \mathrm{z} 60$ is the largest ion with $\mathrm{O}_{3}{ }^{-}$being only $15 \%$ and $\mathrm{O}_{2}^{-}$only $5 \%$ of the intensity of this peak under conditions of discharge current $=$ $-3 \mu \mathrm{A}$, curtain flow of $400 \mathrm{~mL} / \mathrm{min}$, auxiliary flow of $400 \mathrm{~mL} / \mathrm{min}$ and nebulizer flow of $100 \mathrm{~mL} / \mathrm{min}$. 


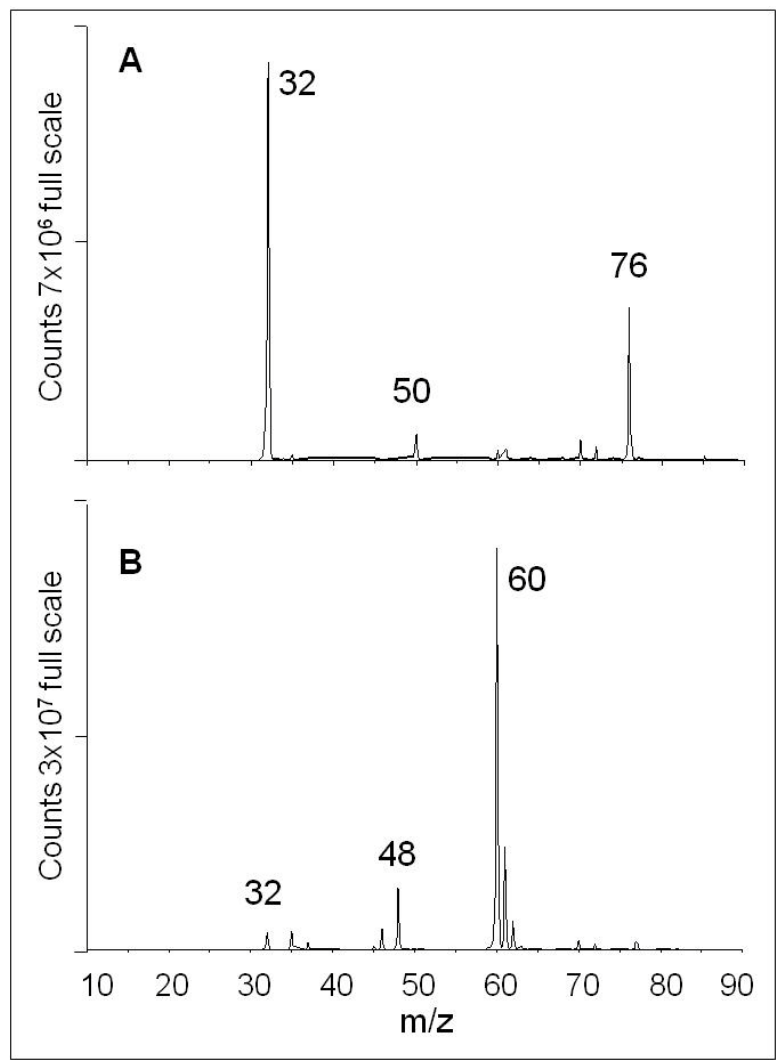

Figure 2.1: Mass spectra of background ions generated $(\mathrm{A})$ in air at atmospheric pressure with a ${ }^{63} \mathrm{Ni}$ ionization source and (B) from a corona discharge ionization source in synthetic air at atmospheric pressure.

A variety of conditions for corona discharge, including discharge current and gas flow rates, were varied to observe the effects on the relative abundances of the ions formed. The ranges for conditions are as follows: discharge current $1 \mu \mathrm{A}$ to $5 \mu \mathrm{A}$; curtain gas $300 \mathrm{~mL} / \mathrm{min}$ to $2000 \mathrm{~mL} / \mathrm{min}$; and sample gas $800 \mathrm{~mL} / \mathrm{min}$ to $5600 \mathrm{~mL} / \mathrm{min}$ which was the sum of the auxiliary and nebulizer gasses. Ion intensities at masses of $32,46,48$, $50,60,62$, and 76 were monitored under the various conditions. In almost all cases $\mathrm{m} / \mathrm{z}$ 60 was the most prevalent ion. The relative intensities of the ions varied with changes to the flow and the discharge current. For example, with the sample gas fixed at $800 \mathrm{~mL} / \mathrm{min}$ and the discharge set at $5 \mu \mathrm{A}$, at low curtain gas flow of $300 \mathrm{~mL} / \mathrm{min}$ ions at $\mathrm{m} / \mathrm{z} 46,48$ and 62 were about as intense as the 60 ion. However, as the curtain gas flow increased 
from 300 to $2000 \mathrm{~mL} / \mathrm{min} \mathrm{m} / \mathrm{z} 48,6,2$ and 46 decreased relative to the 60 ion. This is likely due to the dilution of ozone and $\mathrm{NO}_{\mathrm{x}}$ produced in the discharge with increased flows. Similar observations were noted by Ross and Bell [15]. The opposite behavior was noted upon increasing the discharge current while gas flows were held constant yielding a subsequent increase in $\mathrm{m} / \mathrm{z} 46,48$ and 62 . Previous studies with a dielectric barrier discharge source showed that enclosing the source caused a shift in the predominant ion from $\mathrm{m} / \mathrm{z} 60$ to 62 indicating the formation the nitrate ion [26]. It was postulated that placing the source in an enclosure allowed concentrations of ozone and $\mathrm{NO}_{\mathrm{x}}$ to increase which produced nitrate as the principal ion. In APCI chemistry, the ions that appear range from $\mathrm{m} / \mathrm{z} 32$ to 60 to 62 and intensities of these ions depend upon experimental conditions. However, in commercial APCI mass spectrometers using a point-to-plane corona discharge in clean air, the ion at $\mathrm{m} / \mathrm{z} 60$ appears to dominate the spectra.

Knowing the identity of the $\mathrm{m} / \mathrm{z} 60$ is important for understanding the subsequent ionization of analytes. One possible way of identifying the peak at $\mathrm{m} / \mathrm{z} 60$ would be to look at the isotopic abundances of $\mathrm{C}, \mathrm{N}$, and $\mathrm{O}$ by measuring the ratios of $\mathrm{M}, \mathrm{M}+1$, and $\mathrm{M}+2$ to determine the number of carbon, nitrogen and oxygen atoms present. If the ion at $\mathrm{m} / \mathrm{z} 60$ was $\mathrm{CO}_{3}{ }^{-}$then values (percent of the ion at $\mathrm{m} / \mathrm{z} 60$ ) of $\mathrm{m}+1=1.19 \%$ and $\mathrm{m}+2=0.62 \%$ would be expected. If the ion was $\mathrm{N}_{2} \mathrm{O}_{2}{ }^{-}$then values of $\mathrm{m}+1=0.81 \%$ and $\mathrm{m}+2=0.41 \%$ would be expected. Actual values of $\mathrm{M}+1$ and $\mathrm{M}+2$ were $25.63 \%$ and $6.89 \%$, respectively, as shown in Figure 2.1B, which are much higher percentages than those predicted from isotopic abundances alone and must result from contributions of other ionic species such as $\mathrm{NO}_{3}{ }^{-}$at $m / z 62$. 
Collisionally induced dissociation in a tandem mass spectrometer was another method attempted to elucidate the structure and composition of $m / z$ 60. It was hoped that the daughter ions would give insight into the identity of the parent ion. As shown in Figure 2.2, the only ion produced from the fragmentation of $m / z 60$ is the ion at $m / z 16$, $\mathrm{O}^{-}$ion. Although this provided some information, it did not help in identifying the ion at $\mathrm{m} / \mathrm{z} 60$ since it is possible that this ion could be generated from the fragmentation of either $\mathrm{CO}_{3}{ }^{-}$or $\mathrm{N}_{2} \mathrm{O}_{2}{ }^{-}$.

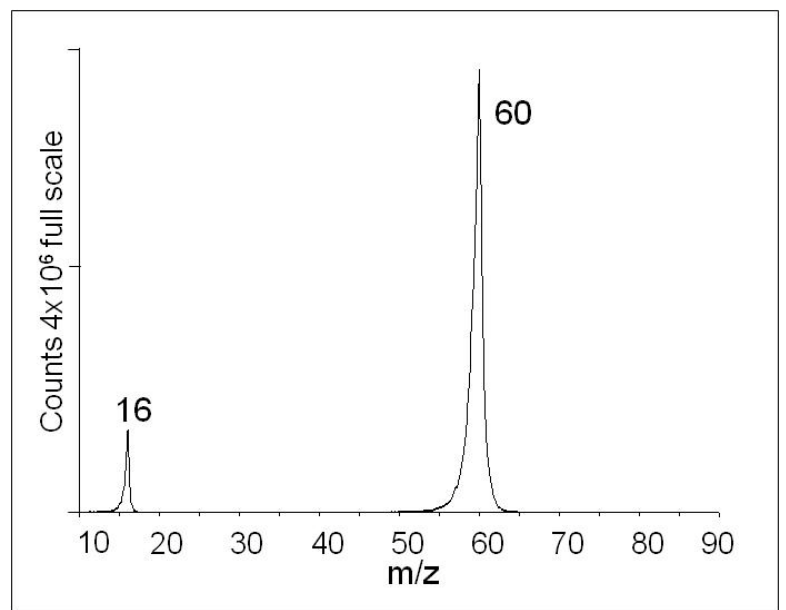

\section{Figure 2.2: MS/MS of collision induced dissociation of $\mathrm{m} / \mathrm{z} 60$ showing only fragment ion of $16\left(\mathrm{O}^{-}\right)$.}

Since the creation of the various ions occurs in air, predominantly containing nitrogen and oxygen, the use of isotopically labeled oxygen or nitrogen was considered. Upon using ${ }^{18} \mathrm{O}$ labeled oxygen, the ion at $\mathrm{m} / \mathrm{z} 60$ would shift two mass units for every ${ }^{18} \mathrm{O}$ atom present in the ion. Due to the cost of this gas and the high flow rates normally used in the API source, the flows were modified in an attempt to use as little oxygen as possible and yet maintain the same observed ionization chemistry. Zero air was replaced with UHP nitrogen and the source was purged with nitrogen (at a flow rate of $2 \mathrm{~L} / \mathrm{min}$ ) 
while monitoring the mass spectra. After several minutes, the intensities of all ions began to decrease. The purge with nitrogen was allowed to continue until the ion signal had dissipated ( $4 \times 10^{4}$ counts compared to $5 \times 10^{7}$ counts for a typical spectrum). At this point it was presumed that the majority of oxygen had been removed and only electrons were present. Oxygen was then metered into the ionization region through the nebulizer gas port. The level was slowly increased and it was noted that significant ion signal was observed at a flow rate of $5 \mathrm{~mL} / \mathrm{min}$ of oxygen. With a flow rate of $2000 \mathrm{~mL} / \mathrm{min}$ of nitrogen, the oxygen concentration was about $0.25 \%$. The mass spectrum with these flow rates is shown in Figure 2.3A and appeared similar to the ionization of air in a corona discharge as observed in Figure 2.1B. The ion at $m / z 60$ is by far the dominant ion present. This experiment was then repeated with the exception of replacing the regular oxygen cylinder with one containing ${ }^{18} \mathrm{O}_{2}$. With the addition of $5 \mathrm{~mL} / \mathrm{min}$ of ${ }^{18} \mathrm{O}$ labeled oxygen, the peaks shifted from $\mathrm{m} / \mathrm{z} 32,48$ and 60 to $\mathrm{m} / \mathrm{z} 36,54$ and 62 corresponding to ${ }^{18} \mathrm{O}_{2}^{-},{ }^{18} \mathrm{O}_{3}{ }^{-}$and $\mathrm{CO}_{2}{ }^{18} \mathrm{O}^{-}$as shown in Figure 2.3B. The relative ion abundances resulting from both the labeled and unlabeled oxygen are essentially identical with the shifts in mass due to the labeled oxygen. From this data it is presumed that $\mathrm{m} / \mathrm{z} 60$ is $\mathrm{CO}_{3}{ }^{-}$, where the ionization occurs with an ion and neutral $\mathrm{CO}_{2}$ thus providing only one labeled oxygen atom. The formation of $\mathrm{CO}_{3}{ }^{-}$likely comes from a reaction between $\mathrm{O}_{3}{ }^{-}$and $\mathrm{CO}_{2}$ as a result of ozone which is generated in the electric discharge. 


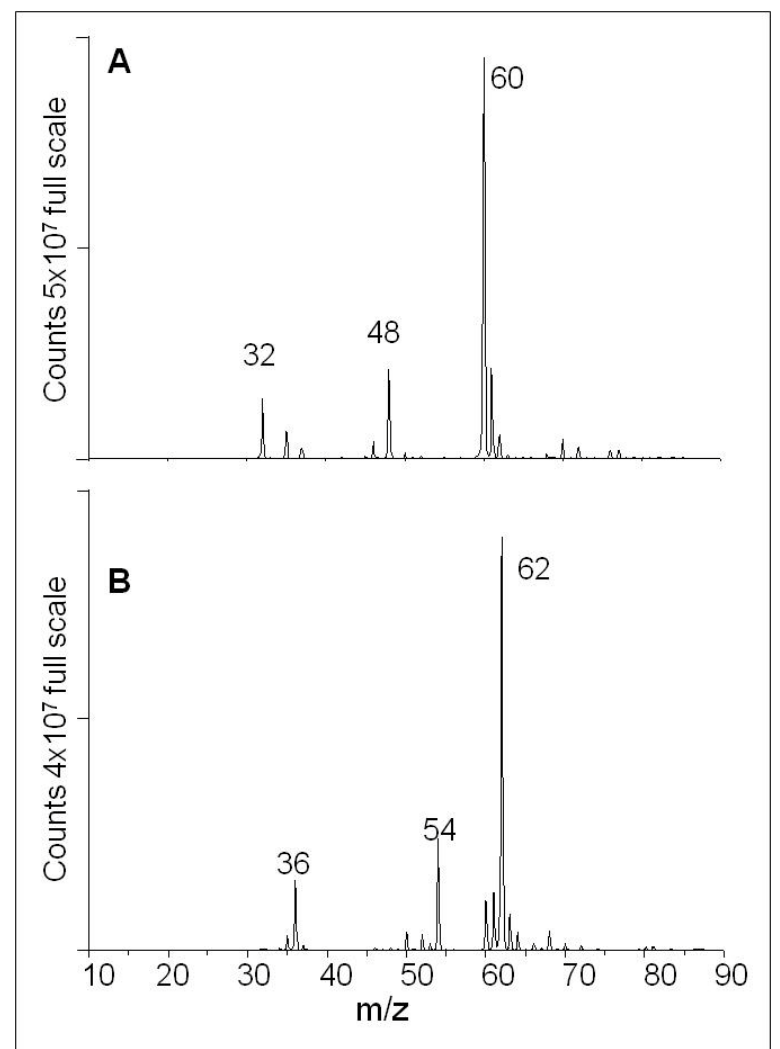

Figure 2.3: Mass spectra of the corona discharge with $\mathrm{N}_{2}$ and $5 \mathrm{~mL}$ of oxygen (A) with regular oxygen and $(B)$ with ${ }^{18} \mathrm{O}$ labeled oxygen.

The corona needle was replaced by the ${ }^{63} \mathrm{Ni}$ source while maintaining the same flows using labeled oxygen. Figure 2.4 shows the results of using ${ }^{18} \mathrm{O}$ labeled oxygen with the ${ }^{63} \mathrm{Ni}$ ionization source. As expected, the ions at $\mathrm{m} / \mathrm{z} 32,50$ and 76 shifted to $\mathrm{m} / \mathrm{z}$ 36, 54 and 80 corresponding to ${ }^{18} \mathrm{O}_{2}^{-}, \mathrm{H}_{2} \mathrm{O}^{18} \mathrm{O}_{2}{ }^{-}$and $\mathrm{CO}_{2}{ }^{18} \mathrm{O}_{2}^{-}$. The $\mathrm{CO}_{2}$ is likely present as an impurity in the gas streams themselves or from small diffusion from the surrounding room air. The presence of $\mathrm{CO}_{2}$ even in relatively pure gas streams is expected at small levels (low $\mathrm{ppm}_{\mathrm{v}}$ range), similar to low $\mathrm{ppm}_{\mathrm{v}}$ amounts of water in zero air. Globally averaged concentration of $\mathrm{CO}_{2}$ in air is around $383 \mathrm{ppm}_{\mathrm{V}}[27]$ and is generally higher in urban areas and room air can be up to 10 times higher than 
background levels, thus the presence of a $\mathrm{CO}_{2}$ is expected and is attributed to the formation of $\mathrm{CO}_{3}{ }^{-}$.

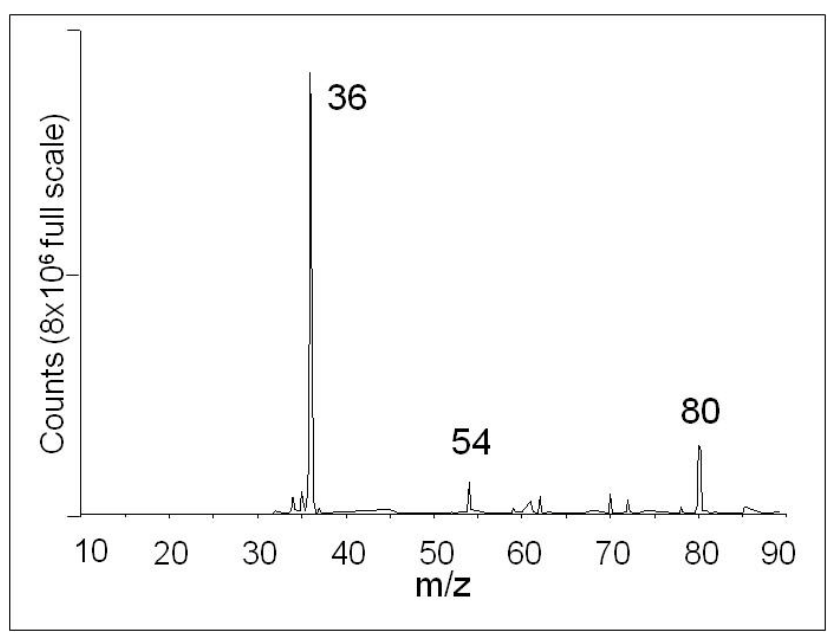

Figure 2.4: Mass spectrum of ${ }^{63} \mathrm{Ni}$ with ${ }^{18} \mathrm{O}_{2}$.

In order to confirm the ion identity of $\mathrm{m} / \mathrm{z} 60$ as $\mathrm{CO}_{3}{ }^{-}$, MS/MS fragmentation was performed on $\mathrm{m} / \mathrm{z} 62$ that was formed with the labeled oxygen in the corona discharge. Fragment ions were produced at $\mathrm{m} / \mathrm{z} 16$ and 18 as shown in Figure 2.5. The abundance of $\mathrm{m} / \mathrm{z} 16$ to 18 is approaching a 2:1 ratio, inferring the identity as $\mathrm{C}^{16} \mathrm{O}_{2}{ }^{18} \mathrm{O}^{-}$. In contrast, fragmentation of $\mathrm{NO}_{3}{ }^{-}(\mathrm{m} / \mathrm{z} 62)$ produced different ions at $\mathrm{m} / \mathrm{z} 32$ and 46 which are $\mathrm{O}_{2}{ }^{-}$ and $\mathrm{NO}_{2}{ }^{-}$. This indicates that the ion at $\mathrm{m} / \mathrm{z} 62$ formed in a corona discharge with labeled oxygen is different than the nitrate ion formed at $\mathrm{m} / \mathrm{z} 62$ in a corona discharge in air. This fragmentation pattern can be compared to the fragmentation displayed in Figure 2.2 of $\mathrm{m} / \mathrm{z} 60$ which shows only $\mathrm{m} / \mathrm{z} 16$ as a fragment. These fragmentation patterns along with the use of ${ }^{18} \mathrm{O}$ labeled oxygen confirmed the identity of the $\mathrm{m} / \mathrm{z} 60$ ion as $\mathrm{CO}_{3}{ }^{-}$, likely produced from a reaction between ${ }^{18} \mathrm{O}_{3}{ }^{-}$and $\mathrm{CO}_{2}$. 


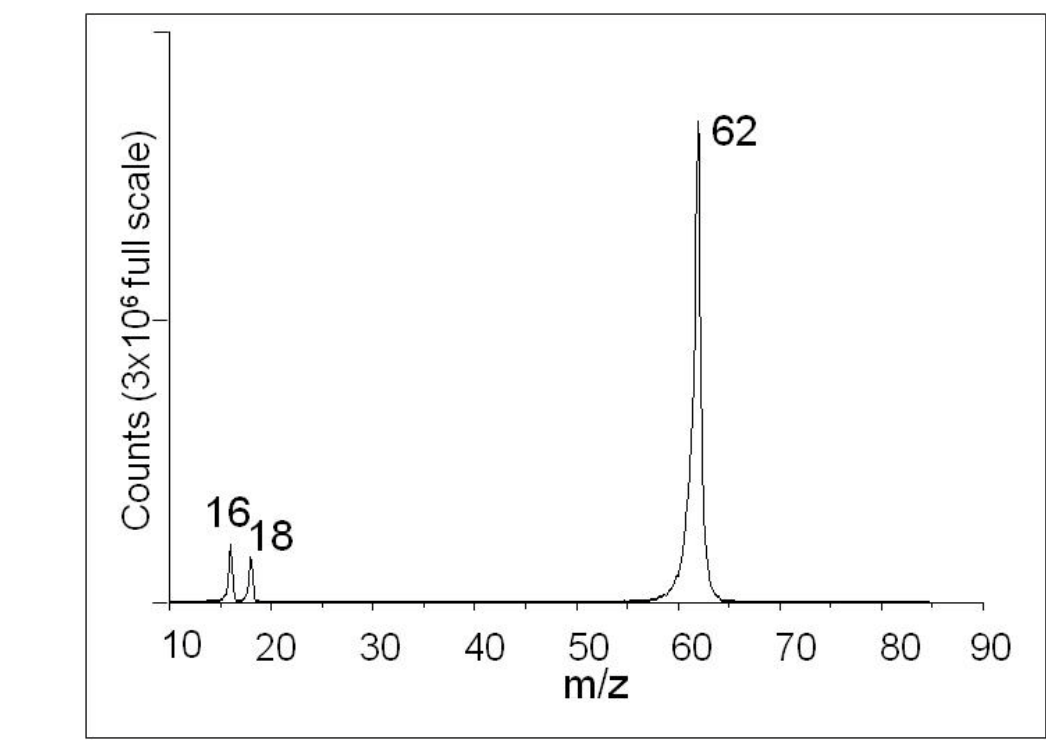

Figure 2.5: MS/MS of the 62 ion from the ${ }^{18} \mathrm{O}$ labeled oxygen.

To emphasize the importance of understanding the reactant ion chemistry and its role on product ion formation, the ionization of nitroglycerin (NG) was explored with a variety of reactant ions. For example with a distributed plasma ion source (DPIS) the only reactant ion was nitrate ion. Upon the introduction of $\mathrm{NG}$ to the ionization region an adduct between Nitrate and NG was formed as shown in Figure 2.6A. The spectrum only contains $\mathrm{NO}_{3}{ }^{-}$at $\mathrm{m} / \mathrm{z} 62$ and NG$\cdot \mathrm{NO}_{3}{ }^{-}$at $\mathrm{m} / \mathrm{z} 289$. With a point to plane corona where the main ion was $\mathrm{CO}_{3}{ }^{-}$, addition of $\mathrm{NG}$ provided a more complex spectrum displayed in Figure 2.6B. In this spectrum many ions are evident including: $\mathrm{NO}_{2}{ }^{-}, \mathrm{CO}_{3}{ }^{-}, \mathrm{NO}_{3}{ }^{-}$and adducts NG. $\mathrm{CO}_{3}{ }^{-}$and NG$\cdot \mathrm{NO}_{3}{ }^{-}$. Although $\mathrm{CO}_{3}{ }^{-}$is the predominant reactant ion, $\mathrm{O}_{2}{ }^{-}$and $\mathrm{O}_{3}{ }^{-}$are also present and thought to interact with $\mathrm{NG}$ to produce $\mathrm{NO}_{2}{ }^{-}$and $\mathrm{NO}_{3}{ }^{-}$ observable in the spectrum. When NG was added in the presence of a chloride ion, the NG. $\mathrm{Cl}^{-}$adduct was formed, as shown in Figure 2.6C, at m/z 262 and 264. Nitrate and nitrite ions are also observed in the presence of the chloride reactant ion, and thought to result from complex, multistep ionization processes occurring in the discharge region. 


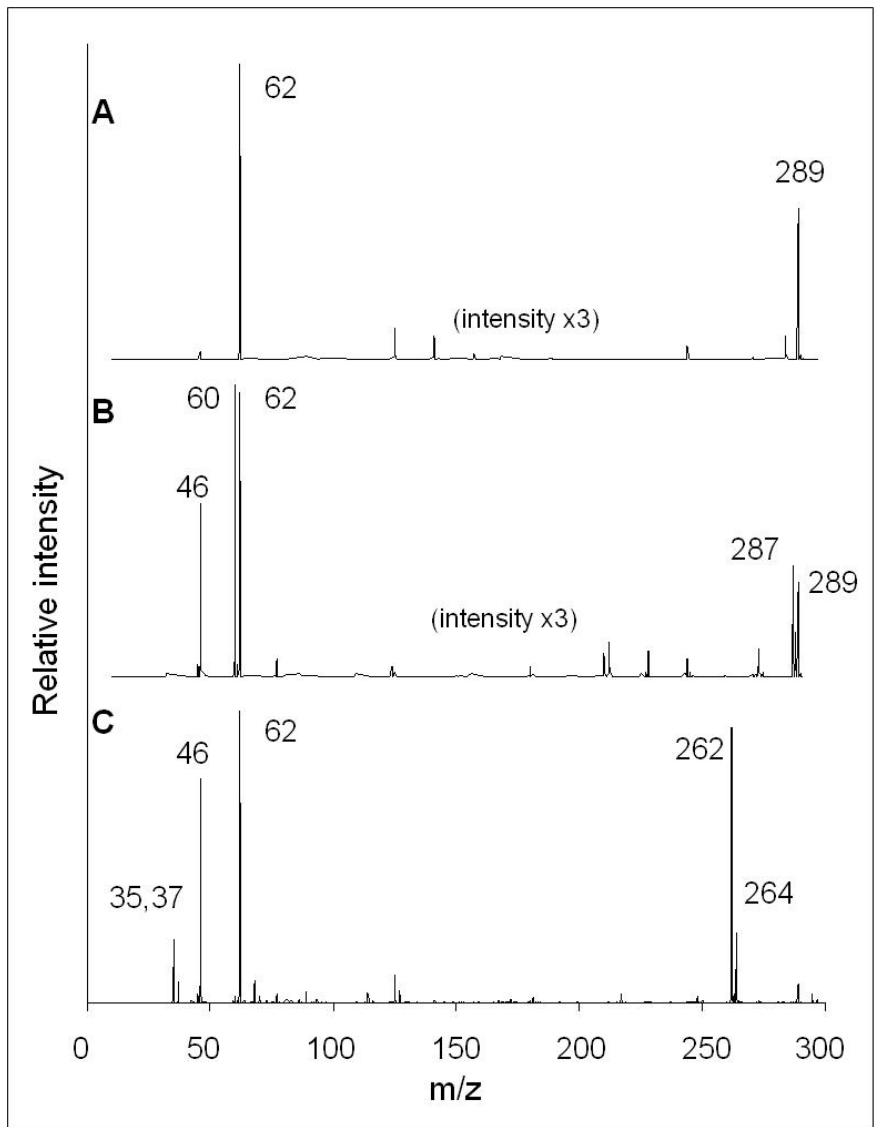

Figure 2.6: Mass spectra of nitroglycerine (A) from a DPIS ionization source, (B) from a corona discharge ionization source and (C) from a corona discharge with carbon tetrachloride.

Collision induced dissociation of the adducts of $\mathrm{NO}_{3}{ }^{-}, \mathrm{CO}_{3}{ }^{-}$and ${ }^{35} \mathrm{Cl}^{-}$with $\mathrm{NG}$ were performed to investigate the fragment ions produced. These MS/MS spectra are displayed in Figure 2.7. Fragmentation of $\mathrm{NG} \cdot \mathrm{CO}_{3}{ }^{-}$produces $\mathrm{m} / \mathrm{z} 60(\mathrm{~B})$ where fragmentation of $\mathrm{NG} \cdot \mathrm{NO}_{3}{ }^{-}$produces the $\mathrm{NO}_{3}{ }^{-}$ion (A). The fact that $\mathrm{CO}_{3}{ }^{-}$is the only fragment of the $\mathrm{NG} \cdot \mathrm{CO}_{3}{ }^{-}$adduct may indicate a high electron affinity of $\mathrm{CO}_{3}{ }^{-}$, ranking it close to that of $\mathrm{NO}_{3}{ }^{-}(3.7-3.9 \mathrm{eV})$. In the NIST Webbook [18], electron affinity values for $\mathrm{CO}_{3}{ }^{-}$range from 1.8-3.5 eV with later references indicating higher values. The fragmentation pattern here indicates the charge resides with $\mathrm{CO}_{3}{ }^{-}$and tends to support the 
high values listed. It is noteworthy that under similar fragmentation conditions, the chloride adduct fragments into multiple ions, $\mathrm{NO}_{3}{ }^{-}, \mathrm{NO}_{2}{ }^{-}$and $\mathrm{Cl}^{-}$, with $\mathrm{NO}_{3}{ }^{-}$being the largest fragment peak (C). In IMS studies [28] it was shown that the chloride adduct of $\mathrm{NG}$, when heated in an IMS above about $130^{\circ} \mathrm{C}$, fragments to only provide the nitrate ion. This was believed to be due to the high electron affinity of the nitrate (higher than that of chloride). However the fragmentation pattern shown here is different, possibly indicating a different mechanism i.e. in the IMS studies fragmentation occurs via thermal decomposition and here it is collisionally induced. This indicates that direct comparisons between the mass spectral data and the IMS data may not be directly correlated.

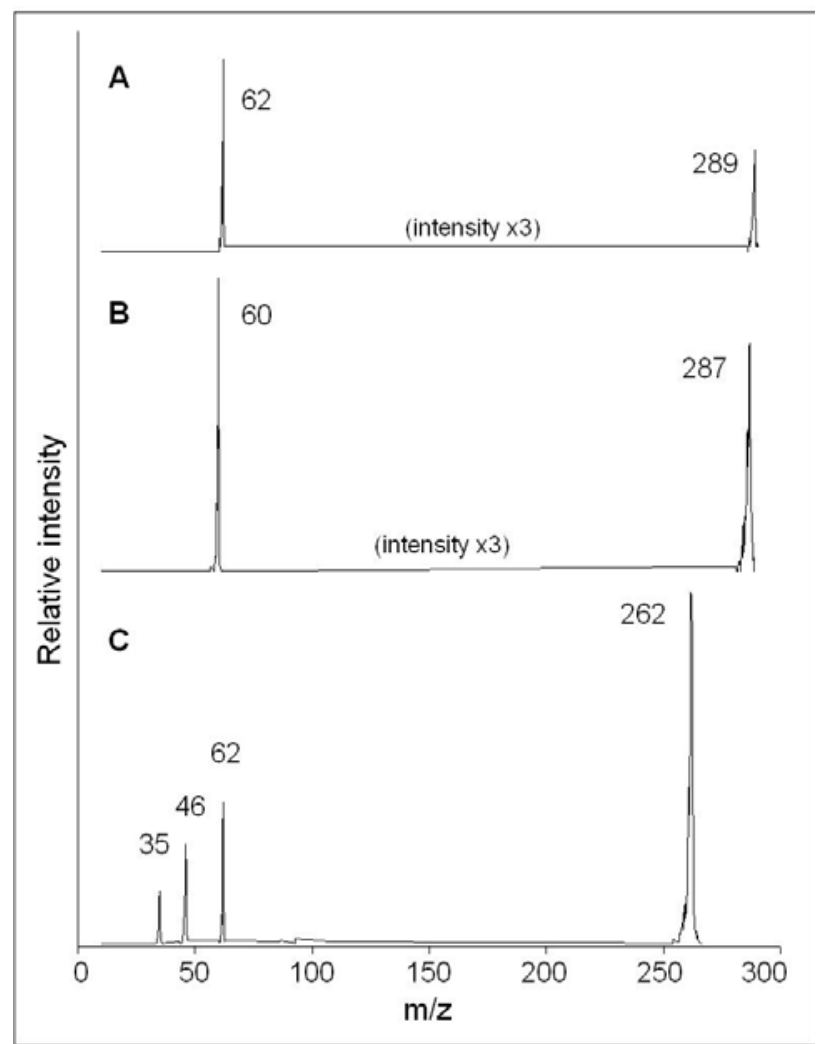

Figure 2.7: MS/MS of nitroglycerine $(\mathrm{mw}=227)(\mathrm{A})$ from a DPIS ionization source $\left(\mathrm{m} / \mathrm{z} 289, \mathrm{NO}_{3}\right.$ adduct), (B) from a corona discharge ionization source $\left(\mathrm{m} / \mathrm{z} 287, \mathrm{CO}_{3}\right.$ adduct) and (C) from a corona discharge with carbon tetrachloride $\left(\mathrm{m} / \mathrm{z} 262,{ }^{35} \mathrm{Cl}^{-}\right.$ adduct). 


\section{CHAPTER 3}

\section{CHARATERIZATION OF A DISTRIBUTED PLASMA IONIZATION SOURCE (DPIS) FOR ION MOBILITY SPECTROMETRY AND MASS SPECTROMETRY}

This current work describes the development of a different type of corona discharge ionization source that involves placing an RF voltage across two electrodes in contact with and on opposite sides of a dielectric. This source is referred to as a distributed plasma ionization source (DPIS) and preliminary results have been presented previously $[29,30]$. A recent publication describes another ionization source that also uses an alternating voltage placed across a dielectric material, referred to as a dielectric barrier discharge ionization source that was developed for surface ionization of explosives [31]. Differences in this configuration are that a solid sample was placed on the dielectric surface attached to a flat copper electrode, and the other electrode, a hollow tube with a gas stream directed at the sample surface, was placed a few millimeters away.

This paper discusses the development of the DPIS that involves placing a highvoltage, alternating current, across a dielectric to produce plasma. Reactant ions formed in the plasma then ionizes sample vapors that are introduced into the ionization region. The potential advantages of this source over a conventional point-to-plane corona discharge are in the longevity of operation and inherent physical stability obtained by fixing both electrodes to a solid surface. This study involves investigations into various configurations of the source, an analysis of the positive and negative reactant ions produced and subsequent ionization of selected compounds characterized by IMS and MS. 


\section{Experimental}

\section{Source design}

The distributed plasma ion source (DPIS) [29,32] can be of any shape and it simply requires two electrodes of dissimilar size separated by a dielectric. The DPIS used in this study was constructed with a $19 \mathrm{~mm}$ X $19 \mathrm{~mm}$ microscope slide cover that was 0.018mm thick (Perfect Parts Company, Baltimore, MD). One side of the slide cover was completely coated with titanium (referred to as the large electrode), while the other side had a $4 \mathrm{~mm}$ disk of titanium in the center (referred to as the small electrode). The titanium was sputtered onto the glass cover to an approximate thickness of $1.2 \mu \mathrm{m}$ (Figure 3.1A). Wires were attached to each electrode and connected to a lab built power supply that provided an approximately $6 \mathrm{kV}$ peak to peak RF voltage at a frequency of $100 \mathrm{kHz}$ and was battery powered allowing the source and electronics to float to the desired DC voltage. Application of this RF voltage across the two electrodes separated by the dielectric caused the production of a plasma that was evident by a blue glow around the outer edge of the small electrode.

The source was operated in two different physical configurations identified as open and enclosed. The open source configuration consisted of only the glass slide cover with wires connected to the electrodes using silver conductive paint (M.G. Chemicals in Toronto, Ont., Canada) and is shown in Figure 3.1A. The glass slide was positioned parallel to the interface plate of the mass spectrometer with the small electrode approximately $1 \mathrm{~cm}$ in front of and facing the instrument's orifice. For the enclosed source configuration, shown in Figure 3.1B, the glass slide cover was placed between a copper washer and a Teflon cylinder $32 \mathrm{~mm}$ o.d., $13 \mathrm{~mm}$ i.d. and $10 \mathrm{~mm}$ thick. The large 
electrode on the slide cover was placed on the copper washer so the small electrode was in the center of the Teflon cylinder. One wire was attached to the bottom of the copper washer via a metal nut and a second wire was fastened to the small electrode with silver conductive paint. The cylinder, source and copper washer were held together by four nylon screws. A 5mm hole was placed into the side of the cylinder and an $8 \mathrm{~mm}$ wedge (shown on the bottom of the cylinder in Figure 3.1) was cut out opposite the hole. The enclosed source was oriented so that the slide cover was perpendicular to the interface plate and the opening of the wedge was $1 \mathrm{~cm}$ away from and facing the orifice. Air flow passed through the hole, across the small electrode and through the wedge towards the orifice of the mass spectrometer.

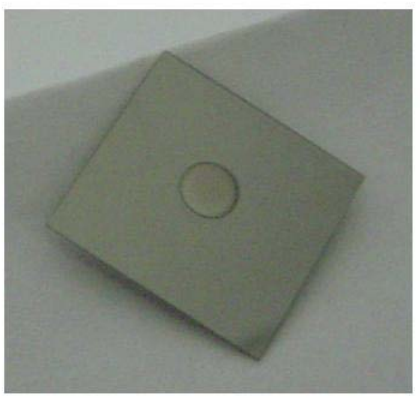

A: Open source

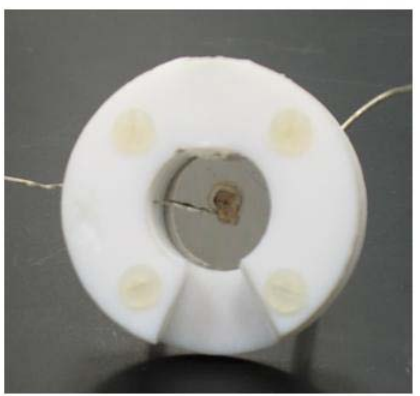

B: Enclosed source

Figure 3.1: The distributed plasma ion source, DPIS consisting of a 19x19 mm microscope slide cover with $1.2 \mu \mathrm{m}$ thick titanium coating completely covering the bottom side (large electrode) and 4mm disc on top (small electrode). (A) the DPIS shown in an open configuration wires were connected to each electrode (B) the enclosed DPIS contained in a Teflon cylinder. 
IMS-QMS

The instrument used in the positive ion study was an ion mobility spectrometer interfaced to a quadrupole mass spectrometer (IMS-QMS) constructed at Washington State University (WSU, Pullman, WA). The basic design of the instrument can be obtained elsewhere $[33,34]$. IMS operating conditions were as follows: IMS drift tube length: $24.7 \mathrm{~cm}$; drift tube temperature: $200^{\circ} \mathrm{C}$; atmospheric pressure: 690 torr; drift gas: $\mathrm{N}_{2}$; drift gas flow rate: $\sim 1000 \mathrm{ml} / \mathrm{min}$; voltage on the target screen $10.48 \mathrm{kV}$; voltage on the ion gate $8.97 \mathrm{kV}$; distance between the target screen and the DPIS $\sim 2 \mathrm{~cm}$. The DPIS was floated to a voltage of $14.0 \mathrm{kV}$

The IMS was interfaced to a model 150-QC ABB Extrel (Pittsburgh, PA) quadrupole MS ( $\mathrm{m} / \mathrm{z}$ range of $0-4000 \mathrm{amu}$ ) via a $40-\mu \mathrm{m}$ pinhole interface. The output signal from the MS detector (electron multiplier) was further amplified by a Keithley model 427 amplifier (Keithley Instruments, Cleveland, $\mathrm{OH}$ ) and then sent to either the MS data acquisition system or IMS acquisition system. Merlin software (ABB Extrel, Pittsburgh, PA) was utilized for all mass spectral analysis and mass spectrometer control. For the IMS gating and data acquisition, the electronic controls were built at Washington State University. The data acquisition and IMS gate-control software employed was Labview-based (National Instruments, Austin, TX, PC Card\# PCI-M01-16XE-10, Windows 2000) and modified at Washington State University. Ion mobility spectra were obtained in two ways: full scan or single ion monitoring (SIM). In the full scan mode of operation an IMS spectrum of all ions generated is obtained with the MS serving as an ion transfer region between the IMS and the detector. In the SIM mode of operation, an 
IMS spectrum of preselected ions is obtained with the MS used as an ion filter for ions of specific $m / z$ between the IMS and the detector.

\section{APCI/MS/MS}

The mass spectrometer used to investigate the DPIS in the negative ion mode was a PE Sciex API-III triple quadrupole mass spectrometer (Thornhill, Ont., Canada) with a point-to-plane corona discharge ionization source. The corona discharge needle was removed from the factory supplied heated nebulizer APCI source. The DPIS was interfaced to the mass spectrometer by pressing the $5 \mathrm{~mm}$ hole in the Teflon housing of the DPIS around the glass tube protruding from the heated nebulizer. Sample flow from the heated nebulizer passed across the surface of the DPIS towards the inlet of the mass spectrometer. The DPIS was floated to -400 VDC below the interface voltage so that anions would move from the source towards the mass spectrometer. Typical interface voltages were between -300 and -600 VDC.

IMS

The ion mobility spectrometer used in the negative ion study was a Phemto-Chem MMS 160 IMS (PCP Inc, West Palm Beach, FL). Operating conditions were as follows: IMS reaction region: $5 \mathrm{~cm}$; drift tube length: $10 \mathrm{~cm}$; drift tube temperature: $25.6^{\circ} \mathrm{C}$; atmospheric pressure: 646 torr; drift gas: purified air; drift gas flow rate: $550 \mathrm{~mL} / \mathrm{min}$; electric field: $200 \mathrm{~V} / \mathrm{cm}$; DPIS floating at $3000 \mathrm{VDC}$; The DPIS was placed about $2 \mathrm{~cm}$ in front of the reaction region. 


\section{Chemicals}

UHP Nitrogen (99.999\% pure) obtained from Matheson Tri-gas, Albuquerque, NM was used as the plenum gas in the Sciex MS. Purified air obtained from a Zero Air Generator (Puregas, Broomfield, CO) was used as the nebulizer gas for the APCI/MS/MS and for the drift gas in the PCP IMS. In the positive ion investigations, preheated counter flowing drift gas $\left(\mathrm{N}_{2} ; 99.99 \%\right.$ pure $)$ at a flow rate of $\sim 900 \mathrm{~mL} / \mathrm{min}$ was introduced at the end of the IMS drift region after passing through a moisture trap (Aldrich Chemical Co. Inc. (Milwaukee, WI, 53201, U.S.A.).

RDX, PETN and NG standards were obtained from Cerilliant (Round Rock, TX) at $1000 \mu \mathrm{g} / \mathrm{mL}$ in acetonitrile and working solutions were diluted in methanol to 1:10 for concentrations of $100 \mathrm{ng} / \mathrm{uL} .50 \mu \mathrm{L}(5 \mu \mathrm{g})$ of the dilution was added to the heated nebulizer (cold). After the solvent was allowed to evaporate with the temperature off, heat was applied to the nebulizer (to $150^{\circ} \mathrm{C}$ ) to desorb the explosive. Auxiliary and nebulizer flows were at $500 \mathrm{~mL} / \mathrm{min}$ and $100 \mathrm{~mL} / \mathrm{min}$, respectively. Significant quantities of RDX remained and were persistent in the spectra for approximately 10-20 minutes. Each spectrum was the result of approximately 25 scans (averaged). Caffeine, 2,4lutidine and methamphetamine were obtained from Aldrich Chemical Co. Inc. (Milwaukee, WI, 53201, U.S.A.) and were classified as ACS reagent grade with $\geq 99 \%$ purity. 


\section{Results and Discussion}

Electric potential modeling

A particle trajectory modeling program, LORENTZ [35], was used to model cross section of the source configuration. The equipotential voltage lines define field gradient contours within the DPIS and are shown in Figure 3.2. Simulation shows that the contours cluster at the interface between the small electrode (right side) and the dielectric, indicating the largest electric field gradient at the edge of the small electrode. This was experimentally observed by a blue glow of the discharge occurring around the edge of the small electrode.

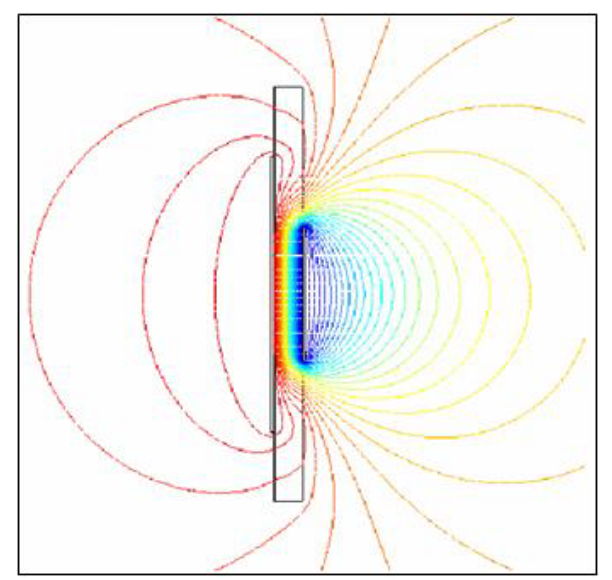

Figure 3.2: A side view of the electric field gradient simulation of the DPIS with the large electrode on the left side and the small electrode on the right. Lines show the equipotential voltage contours produced by the source.

Physical stability with extended use

Testing was performed to see how prolonged use of the DPIS affected the physical aspects and ion production characteristics of the source. The source was operated continuously for three months drawing a current of 40 to $80 \mathrm{~mA}$ using an input voltage of approximately $12 \mathrm{~V}$ to power the $\mathrm{RF}$ generator. This relates to a power consumption of less than 1 watt. Periodically, the source was turned off and 
photographed under a microscope (Leica, DME, Leica Microsystems inc., Buffalo, NY) with an overall magnification of 40x. Figure 3.3 shows the magnified small electrode at 4 intervals encompassing about 1 month of continuous operation: (A) prior to operation, (B) after 6 days, (C) after 16 days, and (D) after 33 days. The small circular electrode, as seen in Figure 3.1, mostly fills the square with an attached wire partially visible from the bottom left with a bead of silver paint as seen at the center. After 6 days of continuous operation, surface deterioration became apparent as seen by the ring surrounding the outer edge of the small electrode where the discharge occurs. Figure 3.3C is a photograph of the source after 16 days of continuous operation; note that the corrosion had increased as evident by the broader ring. After 33 days significant corrosion over the entire surface of the source was observed; however, the source was still producing ions. The source was operated for an additional 2 months and a blue glow was still visible when the experiment was halted. In all our experience with operating various configurations of the DPIS, whenever a blue glow was observed, subsequent ions were detectable by the mass spectrometer. 


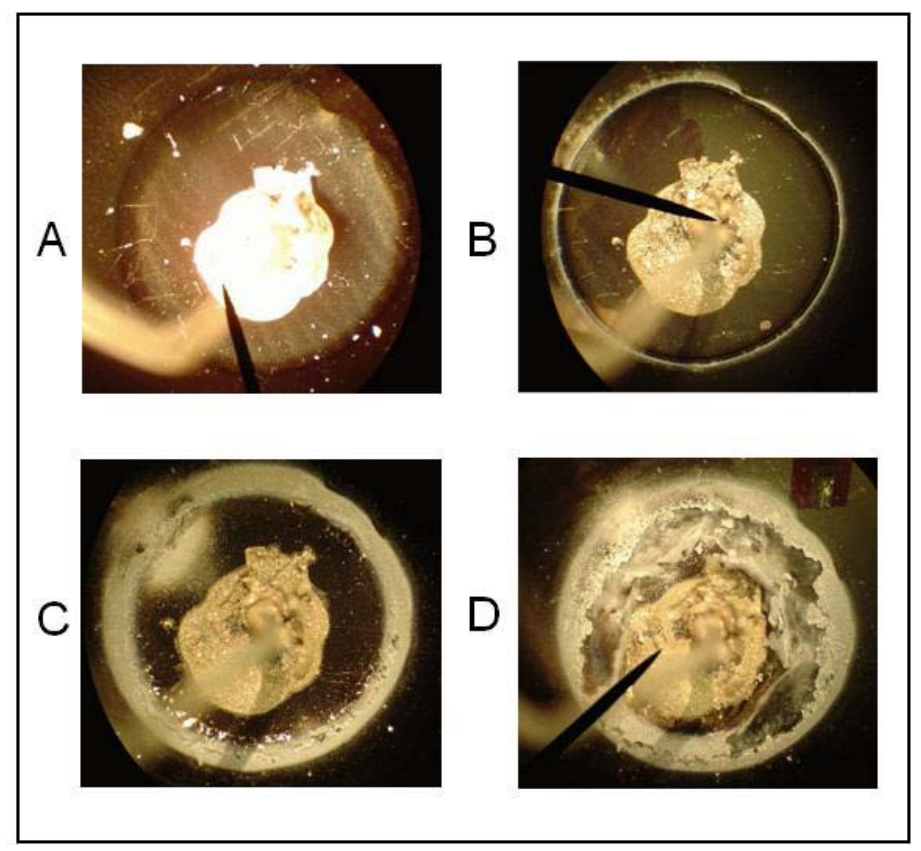

Figure 3.3: Magnified images over 33 days of the continuous operation of the DPIS. Photograph of the small electrode (A) prior to use, (B) after 6 days, (C) after 16 days and (D) after 33 days.

\section{Positive Ions in DPIS}

Ions generated by the open DPIS were introduced into the IMS-QMS and IMS spectra were acquired by operating the quadrupole mass spectrometer in either single ion mode (SIM) or full scan where the quadrupoles were operated in RF only and passed all ions through to the detector. Each IMS spectrum illustrated was an average of 200 IMS spectra. Background ions produced by the ionization of air were mass identified as $\left(\mathrm{H}_{2} \mathrm{O}\right)_{\mathrm{n}} \mathrm{H}^{+}$with $\mathrm{m} / \mathrm{z} 37$ ion $(\mathrm{n}=2)$ as the most abundant ion. Ion mobility spectra of background ions produced by point-to-plane corona discharge (A) and open DPIS (B) are shown in Figure 3.4. Intensity of ions observed by the open DPIS was approximately two-thirds of that observed with corona discharge under identical experimental conditions with the exception of the physical location of the sources. The DPIS was positioned $\sim 2 \mathrm{~cm}$ away from the target screen of the IMS whereas the corona discharge needle was 
$\sim 0.5 \mathrm{~cm}$ away from the target screen. The difference in intensities between the sources could be due to the difference in ion transfer efficiency from the source to the spectrometer because of the relative source-spectrometer distances and/or electric field. The drift time of the positive reactant ions was $13.23 \mathrm{~ms}$ with $\mathrm{K}_{\mathrm{o}}$ value of $2.70 \mathrm{~cm}^{2} \mathrm{~V}^{-1} \mathrm{~s}^{-1}$.

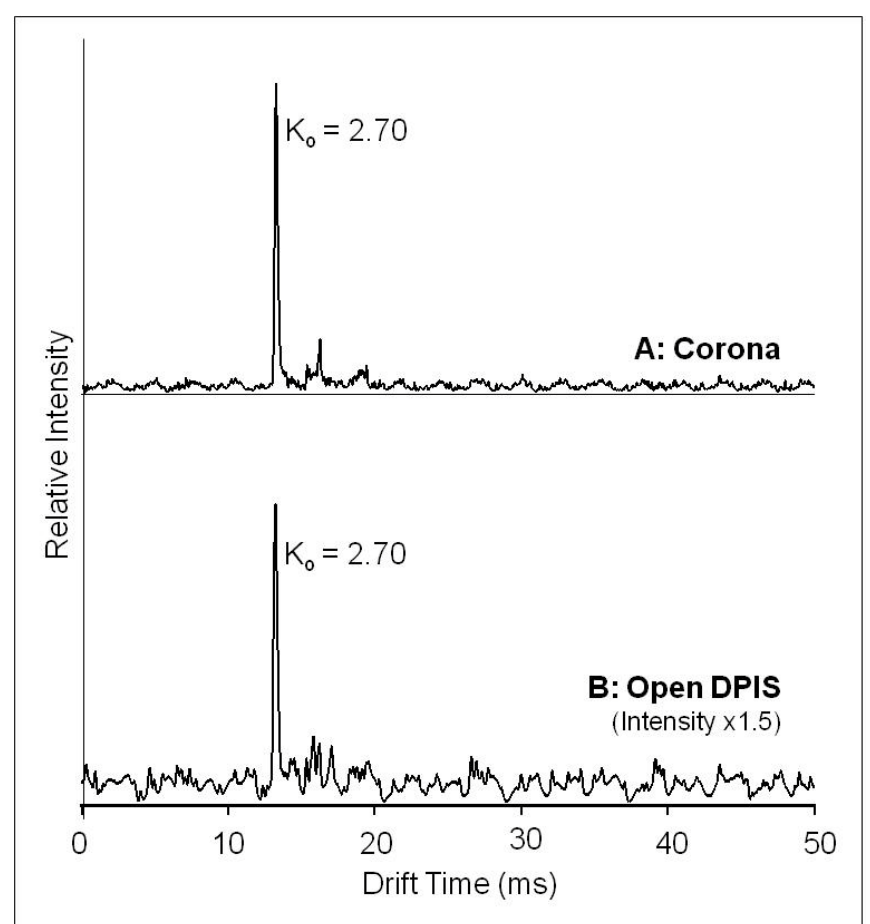

Figure 3.4: Ion mobility spectra of positive background ions produced by (A) corona discharge and (B) open DPIS from ionization of ambient air.

Ionization of 2, 4-lutidine, caffeine and methamphetamine was achieved by positioning a plastic strip ( $3 \mathrm{~mm}$ wide, $1 \mathrm{~mm}$ thickness), on the tip of which each of the analytes were deposited, in the space between the IMS target screen and the ionization source. Figure 3.5 illustrates the ion mobility spectra of 2, 4-lutidine acquired in full scan mode of operation of the IMS-QMS with corona discharge (A) and open DPIS (B). IMS response peaks for reactant ions and protonated 2, 4-lutidine monomer $(\mathrm{m} / \mathrm{z} 108)$ were observed. 


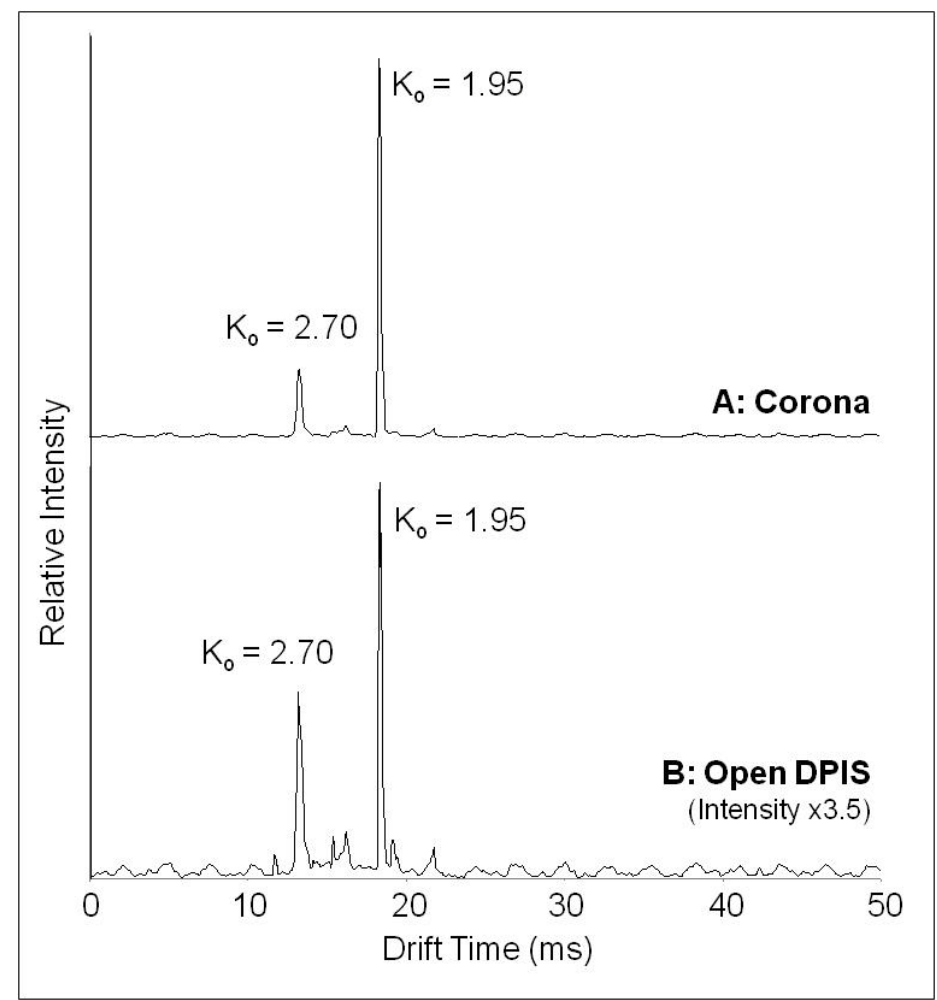

Figure 3.5: Ion mobility spectra of 2, 4-lutidine produced by (A) corona discharge and (B) open DPIS. IMS peaks of the protonated water reactant ions and protonated monomer ions of the 2, 4-lutidine are shown at mobility values of 2.70 and $1.95 \mathrm{~cm}^{2} \mathrm{~V}^{-1} \mathrm{~s}^{-1}$, respectively.

Figure 3.6 shows the ion mobility spectra of caffeine (mol wt: 194), methamphetamine (mol wt: 149) and lutidine (mol wt: 107) acquired in SIM mode of operation with the IMS-QMS and the open DPIS as the ion source. Dominant ions observed in the IMS for caffeine, methamphetamine and lutidine were the protonated molecular ions of each at $m / z$ values of 195,150 , and 108, respectively. Except for an increase in intensities of the peaks, the analytes ionized by corona discharge were similar to those generated by the open DPIS. The reduced mobility values $\left(\mathrm{K}_{\mathrm{o}}\right)$ of the analytes were calibrated against the $\mathrm{K}_{\mathrm{o}}$ value of 2, 4-lutidine as $1.95 \mathrm{~cm}^{2} \mathrm{~V}^{-1} \mathrm{~s}^{-1}$ and were measured to be: caffeine $1.54 \mathrm{~cm}^{2} \mathrm{~V}^{-1} \mathrm{~s}^{-1}$ and methamphetamine $1.63 \mathrm{~cm}^{2} \mathrm{~V}^{-1} \mathrm{~s}^{-1}$. 


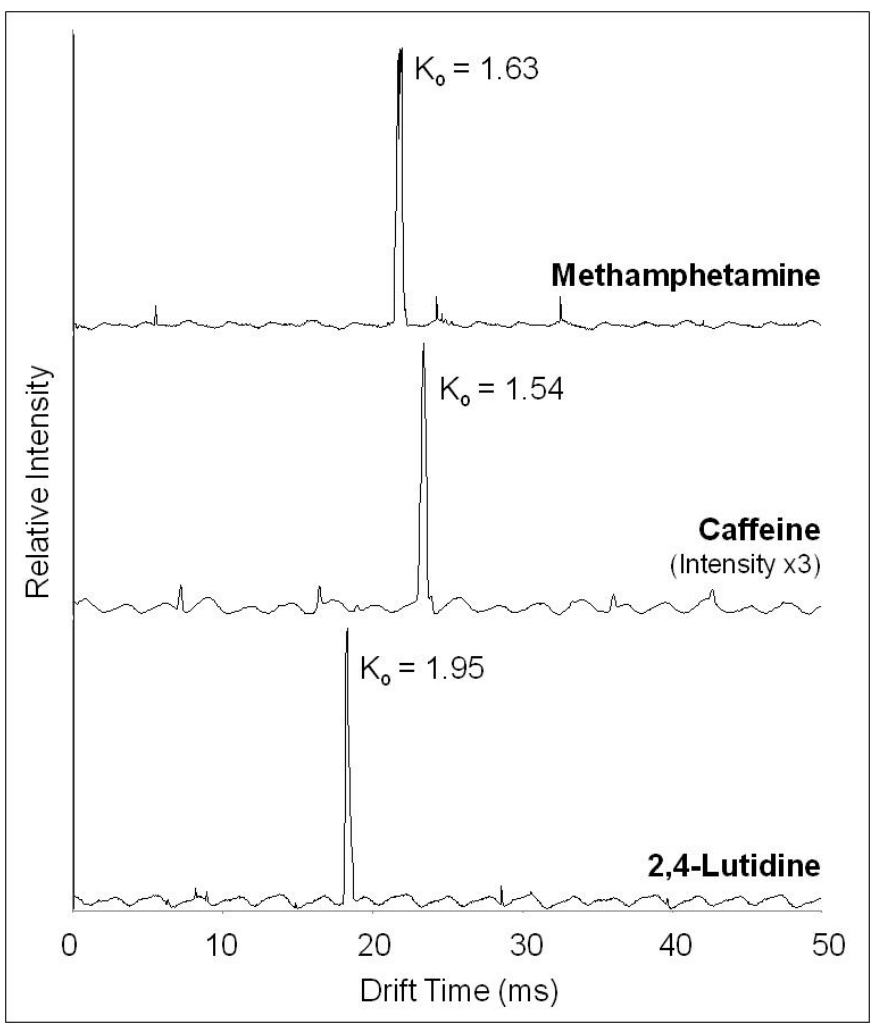

Figure 3.6: Single ion monitored (SIM) ion mobility spectra of methamphetamine $(m / z ~ 150)$, caffeine $(m / z ~ 195)$, and 2 , 4-lutidine $(m / z ~ 108)$ using the open DPIS.

Negative Ions in DPIS

Negative ions generated in clean air were investigated with the API-III mass spectrometer. The negative reactant ions were created using a variety of ionization sources including a ${ }^{63} \mathrm{Ni}$ foil, the factory installed point-to-plane corona discharge, the open DPIS and the enclosed DPIS. The DPIS and ${ }^{63} \mathrm{Ni}$ ionization sources were all floating at about $-400 \mathrm{~V}$ below the interface potential. Representative spectra from each source are shown in Figure 3.7. The atmospheric pressure ionization of air by ${ }^{63} \mathrm{Ni}$ produced $\mathrm{O}_{2}^{-}$and $\mathrm{O}_{2}{ }^{-} \cdot \mathrm{CO}_{2}$. The conventional corona discharge ionization source produced predominantly $\mathrm{CO}_{3}{ }^{-}$in air. The DPIS in an open configuration produced $\mathrm{CO}_{3}{ }^{-}$ ions similar to those from the corona discharge source. The enclosed DPIS source produced mainly $\mathrm{NO}_{3}{ }^{-}$ions. 


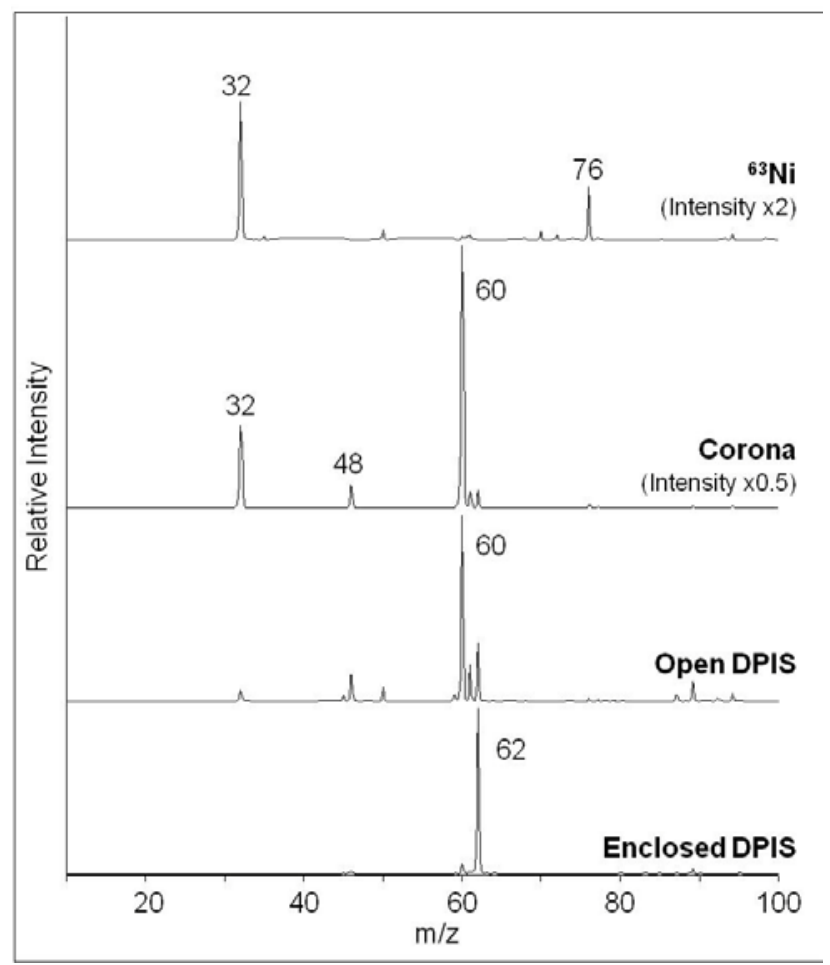

Figure 3.7: Mass spectra of negative ions generated in purified air with ${ }^{63} \mathrm{Ni}$, pointto-plane corona, open DPIS, and enclosed DPIS.

Increasing flow rates to the corona discharge source of an IMS have been shown previously to shift the reactant ion chemistries to favor the production of $\mathrm{O}_{2}^{-}[15,36]$.

Ross and Bell [15], using a reversed high flow (100-500 $\mathrm{mL} / \mathrm{min})$ in a continuous corona discharge in air at atmospheric pressure, showed that concentrations of $\mathrm{O}_{3}{ }^{-}, \mathrm{CO}_{3}{ }^{-}$, and $\mathrm{NO}_{3}{ }^{-}$decreased, and the ions observed were $\mathrm{O}_{2}{ }^{-}$and $\mathrm{O}_{2}{ }^{-} \cdot \mathrm{CO}_{2}$, similar to the reactant ions from a ${ }^{63} \mathrm{Ni}$ source at atmospheric pressure. When the reverse flow rate was reduced to $\leq$ $50 \mathrm{~mL} / \mathrm{min}$ or changed to the forward direction at high flows $(250-500 \mathrm{~mL} / \mathrm{min}) \mathrm{CO}_{3}{ }^{-}$was the predominant ion observed. Other ions produced under these conditions included $\mathrm{O}_{3}{ }^{-}$ and $\mathrm{NO}_{3}{ }^{-}$. Nitrate became the predominant ion at lower forward flow rates (50$200 \mathrm{~mL} / \mathrm{min}$ ). The shift from $\mathrm{O}_{2}{ }^{-}$to $\mathrm{CO}_{3}{ }^{-}$to $\mathrm{NO}_{3}{ }^{-}$occurs due to the buildup of neutrals in the discharge region, including $\mathrm{O}_{3}$ and $\mathrm{NO}_{\mathrm{x}}$, along with other air contaminants such as 
$\mathrm{CO}_{2}$. The formation of $\mathrm{CO}_{3}{ }^{-}$may result from a reaction of $\mathrm{CO}_{2}$ with $\mathrm{O}_{3}{ }^{-}$. Subsequent reactions involving $\mathrm{NO}_{x}$ yield the $\mathrm{NO}_{3}{ }^{-}$ion. With the DPIS in an open configuration, ozone is produced and the $\mathrm{CO}_{3}{ }^{-}$is the predominant ion. The enclosed DPIS allows $\mathrm{NO}_{\mathrm{x}}$ to concentrate in the source thus generating $\mathrm{NO}_{3}{ }^{-}$as the major reactant ion.

Ion mobility spectra of air obtained using a ${ }^{63} \mathrm{Ni}$ source and an enclosed DPIS are shown in Figure 3.8. The reactant ion appearing from the ${ }^{63} \mathrm{Ni}$ source at ambient temperature with a reduced mobility value of $2.17 \mathrm{~cm}^{2} \mathrm{~V}^{-1} \mathrm{~s}^{-1}$ was inferred to be $\mathrm{O}_{2}^{-}$[37]. The reactant ion for the DPIS appeared to the right of the peak generated from the ${ }^{63} \mathrm{Ni}$ source under similar conditions. It had a reduced mobility value of $1.98 \mathrm{~cm}^{2} \mathrm{~V}^{-1} \mathrm{~s}^{-1}$ and was believed to be $\mathrm{NO}_{3}{ }^{-}$as observed with the Sciex mass spectrometer. Other investigations have shown the nitrate peak appearing to the right of the $\mathrm{O}_{2}{ }^{-}$reactant ion [38,39]. In an IMS with an $\mathrm{O}_{2}{ }^{-}$mobility value of $2.57 \mathrm{~cm}^{2} \mathrm{~V}^{-1} \mathrm{~s}^{-1}, \mathrm{NO}_{3}{ }^{-}$was found at 2.46 $\mathrm{cm}^{2} \mathrm{~V}^{-1} \mathrm{~s}^{-1}$ [38]. Although the mobility values reported do not match those given here, the previous values were measured between $110^{\circ} \mathrm{C}$ and $150{ }^{\circ} \mathrm{C}$, and shifts toward higher mobility could be due to desolvation of the reactant ions. The peak intensity in the spectra from the DPIS is 5-6 times lower than that with ${ }^{63} \mathrm{Ni}$ as ion source. This was due to a loss in ion transmission through the DPIS interface to the IMS and not to lower ion currents being generated. Another interesting note is the lack of other peaks in the spectra. This is believed to result from the relatively high electron affinity and non-reactivity of $\mathrm{NO}_{3}{ }^{-}$. The mass spectra of the enclosed DPIS source showed a similar result with $\mathrm{NO}_{3}{ }^{-}$as the dominant peak and relatively few minor peaks compared to the other ionization sources. Since negative ionization processes mainly involve electron transfer, proton abstraction 
or adduct formation between the reactant ion and the analyte, the identity of the reactant ions will impact the ionization of analytes.

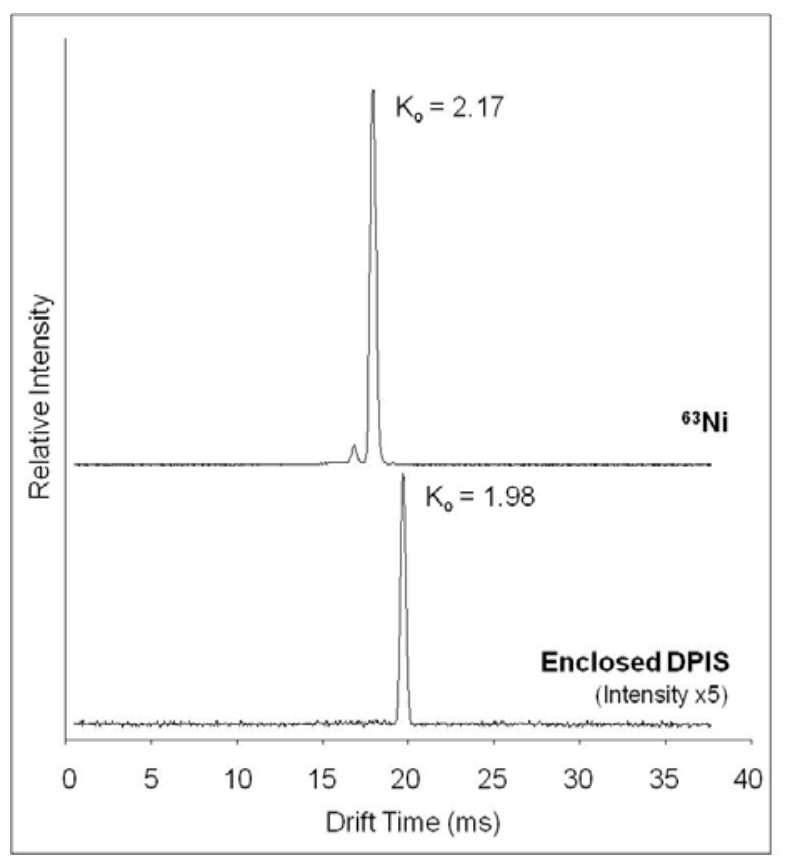

Figure 3.8: IMS spectra of negative ions produced by the atmospheric pressure ionization of clean air at ambient temperature with ${ }^{63} \mathrm{Ni}$ and an enclosed DPIS.

One of the major applications of IMS is in the detection of explosives.

Commercial ion mobility spectrometers utilize a ${ }^{63} \mathrm{Ni}$ source with a chloride dopant for explosives detection. Under these conditions, RDX ionizes by forming an adduct ion $(\mathrm{RDX} \cdot \mathrm{Cl})^{-}$with the chloride ion [40]. In the absence of the chloride dopant, RDX ionizes by first producing $\mathrm{NO}_{2}^{-}$and at higher concentrations of $\mathrm{RDX}$ the $\left(\mathrm{RDX} \cdot \mathrm{NO}_{2}\right)^{-}$adduct is formed [41]. Mass spectrum obtained from the ionization of RDX in the presence of the $\mathrm{CO}_{3}{ }^{-}$reactant ion is shown in Figure 3.9B. The $\mathrm{CO}_{3}{ }^{-}$ion was generated from a corona discharge source. Upon introduction of the RDX $(\mathrm{mw}=222)$ to the corona discharge source the $\mathrm{NO}_{2}{ }^{-}$is formed along with the most intense peak at $\mathrm{m} / \mathrm{z}$ of 268 which is the 
$\left(\mathrm{RDX} \cdot \mathrm{NO}_{2}\right)^{-}$adduct. Small peaks observed at 282, 283 and 284 are likely $\mathrm{CO}_{3}{ }^{-}, \mathrm{HCO}_{3}{ }^{-}$ and $\mathrm{NO}_{3}{ }^{-}$adducts of $\mathrm{RDX}$, respectively. Figure 3.9A is the mass spectra of RDX with an enclosed DPIS source. Upon introduction of RDX, the predominant peak observed at $m / z$ of 284 was the $\left(\mathrm{RDX} \cdot \mathrm{NO}_{3}\right)^{-}$adduct. Low-intensity peaks for $\mathrm{NO}_{2}{ }^{-}$and $\left(\mathrm{RDX} \cdot \mathrm{NO}_{2}\right)^{-}$ions were also detected. Despite the "unreactive" nature of the $\mathrm{NO}_{3}{ }^{-}$reactant ion that suppressed other ion signal in the background, it provided effective ionization for RDX. Other explosives, such as nitroglycerine and PETN, showed similar ionization properties and were observed to form nitrate adducts as well. Although $\mathrm{NO}_{3}{ }^{-}$may not be the optimal reactant ion for all compounds, it does provide further selectivity to the ionization of some explosives.

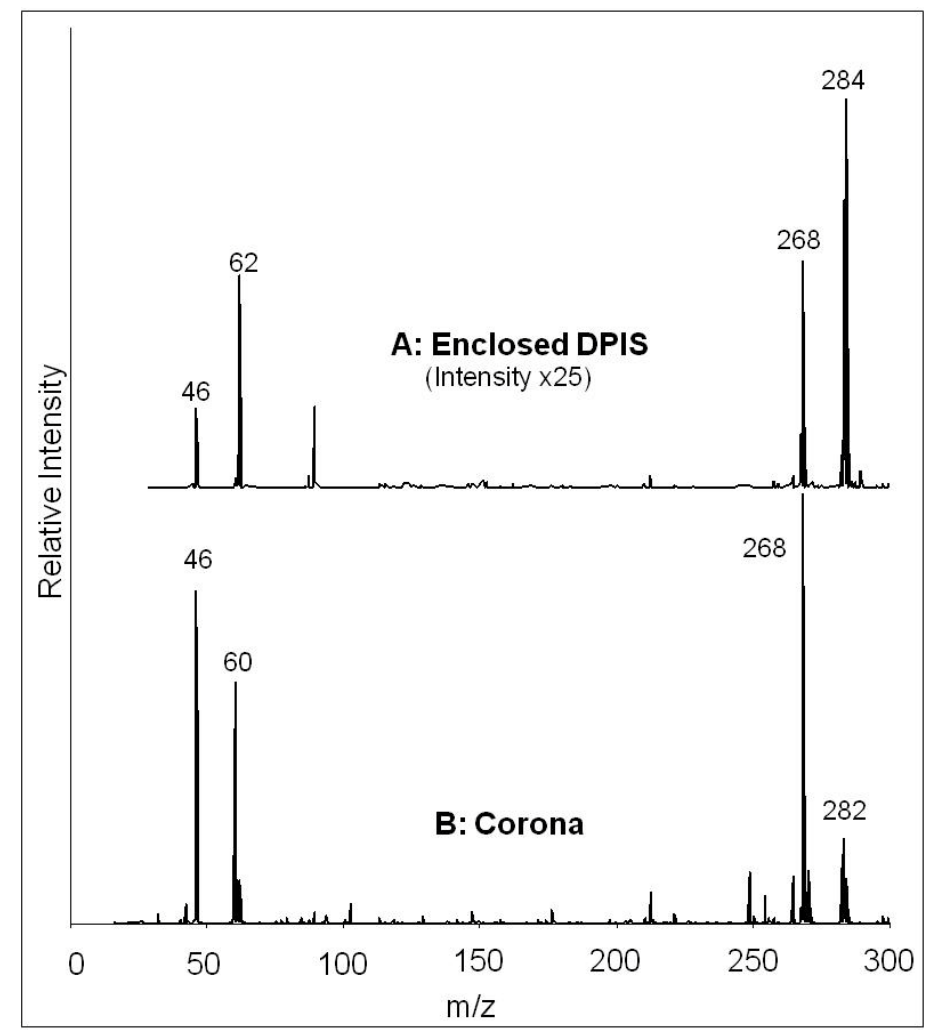

Figure 3.9: Mass spectra of RDX $(\mathrm{mw}=222)$. Ionization of $\mathrm{RDX}$ with the (A) enclosed DPIS and (B) point-to-plane corona discharge. 


\section{CHAPTER 4}

\section{MECHANISMS FOR NEGATIVE REACTANT ION FORMATION IN AN ATMOSPHERIC PRESSURE CORONA DISCHARGE}

APCI of air with a point-to-plane corona discharge in mass spectrometry has been shown to produce $\mathrm{CO}_{3}{ }^{-}$ions as the predominant reactant ion along with smaller amounts of $\mathrm{O}_{3}{ }^{-}, \mathrm{NO}_{2}{ }^{-}, \mathrm{NO}_{3}{ }^{-}$and $\mathrm{O}_{2}{ }^{-}[16,17,42]$. The production of these ions appears to be related to the generation of $\mathrm{NO}_{\mathrm{x}}$ and ozone from a discharge in air. The composition of clean (nonurban) tropospheric air is predominantly nitrogen (78\%), oxygen (21\%) and argon $(0.9 \%)$ and also contains high levels of $\mathrm{CO}_{2}(355 \mathrm{ppm})$ [43]. The primary neutrals generated in a discharge in air include $\mathrm{NO}_{\mathrm{x}}\left(\mathrm{NO} \& \mathrm{NO}_{2}\right)$ and $\mathrm{O}_{3}$, and to a lesser extent $\mathrm{N}_{2} \mathrm{O}$. $[24,44]$ The presence of these neutrals along with electrons generated in the source causes the production of $\mathrm{CO}_{3}{ }^{-}$. As the concentrations of ozone and $\mathrm{NO}_{\mathrm{x}}$ increase, the formation of $\mathrm{NO}_{3}{ }^{-}$ions are observed. The production of $\mathrm{NO}_{3}{ }^{-}$ions was specifically noted in the development of a distributed plasma ion source (DPIS) [26]. When this source was enclosed inside a cylinder, the subsequent ions observed by a mass spectrometer were exclusively $\mathrm{NO}_{3}{ }^{-}$ions.

Since the identity of the reactant ions plays a significant role in the resulting product ions, it is imperative that the ionization processes occurring in the IMS source be well understood and predictable. In an effort to further the use of corona discharge ionization with IMS, a detailed examination of the discharge ion source products was conducted. This was achieved by extracting neutrals generated in a discharge source and introducing them, in a controlled manner, into an IMS containing a ${ }^{63} \mathrm{Ni}$ ionization source. As a consequence, the ion chemistry and desired selectivity could be attained. 
The motivation of this effort was to investigate the ion formation as related to the presence of $\mathrm{NO}_{\mathrm{x}}$ and ozone in the ionization region and relate the appearance of these ions to published reaction rates and gas composition for various pertinent reactions.

\section{Experimental}

\section{Instrumentation}

An ion mobility spectrometer interfaced to a triple quadrupole mass spectrometer IMS/MS/MS model MMS 160 (PCP Inc, West Palm Beach, FL) was used in these experiments and has been described in detail elsewhere [45]. This instrument contains a

${ }^{63} \mathrm{Ni}$ ionization source. Operating conditions were as follows: IMS reaction region: $5 \mathrm{~cm}$; IMS drift tube length: $10 \mathrm{~cm}$; IMS drift tube temperature: $140{ }^{\circ} \mathrm{C}$; IMS pressure: 750 torr; drift gas: air at $600 \mathrm{~mL} / \mathrm{min}$; carrier gas: air at $100 \mathrm{~mL} / \mathrm{min}$; electric field: $3000 \mathrm{~V}$ total or $200 \mathrm{~V} / \mathrm{cm}$; gate width: $200 \mu \mathrm{s}$. The interface region, pumped by a mechanical rough pump, was maintained at a pressure of 280 millitorr. Data collection and instrument control were performed using a PC operating with MS Dos version 6.00 and using KEY 3 version 1.21 software (Keystone Applications). Four types of spectra were obtained with this system including: normal mass spectra (MS); ion mobility spectra with the Faraday plate at the end of the drift region (IMS-F); ion mobility spectra with the electron multiplier (IMS-EM); and ion mobility spectra with selected ion monitoring (IMS-SIM). The mass spectra were collected by operating the IMS with the ion gates fully open. The IMS-F spectra were used to measure drift times and subsequent calculation of the reduced mobility values $\left(\mathrm{K}_{\mathrm{o}}\right)[5]$ reported in this paper. Calculated reduced mobility values measured throughout the experiments remained constant and varied only slightly, 
on the order of $0.01 \mathrm{~cm}^{2} \mathrm{~V}^{-1} \mathrm{~s}^{-1}$. This reproducibility was achieved by maintaining constant temperature and moisture conditions throughout this study. IMS-EM spectra, which used the electron multiplier from the mass spectrometer as the detector while the quadrupoles were operated to pass all ions, produced essentially identical spectra compared with IMS-F with the exception of a shift of approximately $0.5 \mathrm{~ms}$ to longer drift times. This delay is attributed to the time required for the ions to pass through the interface region and the mass spectrometer, and variances in the shift are due to differences in ion mobility. To identify the drift time of an ion with a specific $\mathrm{m} / \mathrm{z}$, an IMS-SIM was collected by setting the quadrupoles to pass a specific $m / z$ while monitoring the drift time. The drift time of this ion could then be compared to the drift times from the IMS-EM and from this correlated to the IMS-F to link $m / z$ to a specific reduced mobility value.

\section{Chemicals / Gases}

Zero air was produced from compressed house air and passed through a Pure Air Generator model CO2RP140 (Domnick Hunter, Charlotte, NC). Manufacturer's specifications were $<1 \mathrm{ppm} \mathrm{CO}_{2},<0.003 \mathrm{ppm}$ non-methane hydrocarbons and a dew point of $-70{ }^{\circ} \mathrm{C}$. The actual water concentration was measured to be $<20 \mathrm{ppm}_{\mathrm{v}}$ as determined by a Moisture Image Series I Hygrometer (GE Panametrics, Billerica, MA). UHP Helium was obtained from Oxarc (Pasco, WA). $\mathrm{O}_{2}(99.998 \%)$ was obtained from Matheson Gas Products, Inc. (Newark, CA). RDX at $0.1 \mathrm{mg} / \mathrm{mL}$ in $50 \%$ methanol $50 \%$ acetonitrile was acquired from AccuStandard (New Haven, CT). 


\section{Experimental Setup}

Neutral vapors $\left(\mathrm{O}_{3}\right.$ and $\left.\mathrm{NO}_{\mathrm{x}}\right)$ generated from a discharge source were introduced at varying concentrations into the IMS containing a ${ }^{63} \mathrm{Ni}$ ionization source using a reactant gas chamber in which the delivery rate of atmospheric gasses to the IMS could be controlled. The ions produced by the ${ }^{63} \mathrm{Ni}$ source were monitored with both the IMS and the MS. The goal of this investigation was to better understand the process in which reactant ions are formed in a discharge ionization source compared to that of ${ }^{63} \mathrm{Ni}$. In order to accomplish this, three separate configurations of the reactant gas chamber were utilized: a Static Container, a Dynamic Container and a Continuous Flow Tube.

The discharge source used for both the Static and Dynamic Container experiments was a distributed plasma ion source (DPIS) described in detail elsewhere [26]. It consisted of a $19 \mathrm{~mm} \times 19 \mathrm{~mm}$ microscope slide cover that was $0.018 \mathrm{~mm}$ thick. One side of the slide cover was completely coated with titanium (large electrode), while the other side had a $4 \mathrm{~mm}$ disk of titanium in the center (small electrode). The titanium was sputtered onto the glass cover to an approximate thickness of $1.2 \mu \mathrm{m}$. Wires were attached to each electrode and connected to a lab built power supply that provided an approximately $6 \mathrm{kV}$ peak to peak RF voltage at a frequency of $100 \mathrm{kHz}$. The glass slide cover was placed between a copper washer and a Teflon cylinder $32 \mathrm{~mm}$ o.d., $13 \mathrm{~mm}$ i.d. and $10 \mathrm{~mm}$ thick. The large electrode on the slide cover was placed on the copper washer so the small electrode was in the center of the Teflon cylinder. The cylinder, source and copper washer were held together by four nylon screws. Although the original design of 
this source was so that it could be located in front of a mass spectrometer, it was used here for generating ozone and $\mathrm{NO}_{\mathrm{x}}$ in air.

The Static Container, shown in Figure 4.1, consisted of a $500 \mathrm{~mL}$ Nalgene bottle with electrical leads that passed through the side of the bottle and connected the DPIS to the power supply. Purified air or oxygen was introduced through a 1/8" Teflon tube near the top of the bottle. The DPIS was operated continuously for specified amounts of time and then gas samples were taken from the bottle for evaluation by a syringe through a small hole near the bottom of the bottle and injected into the inlet of the IMS.

For the Dynamic Container, the Static Container was modified for continuous flow by placing a second $1 / 8$ " tube in the bottom of the bottle shown in part b of Figure 4.1. Purified air was added through the tube in the top of the bottle and the effluent out the bottom that was connected to the inlet of the IMS. This allowed for continuous monitoring of gases produced during operation of the DPIS, which was operated at a $50 \%$ duty cycle (5 s on $5 \mathrm{~s}$ off). 


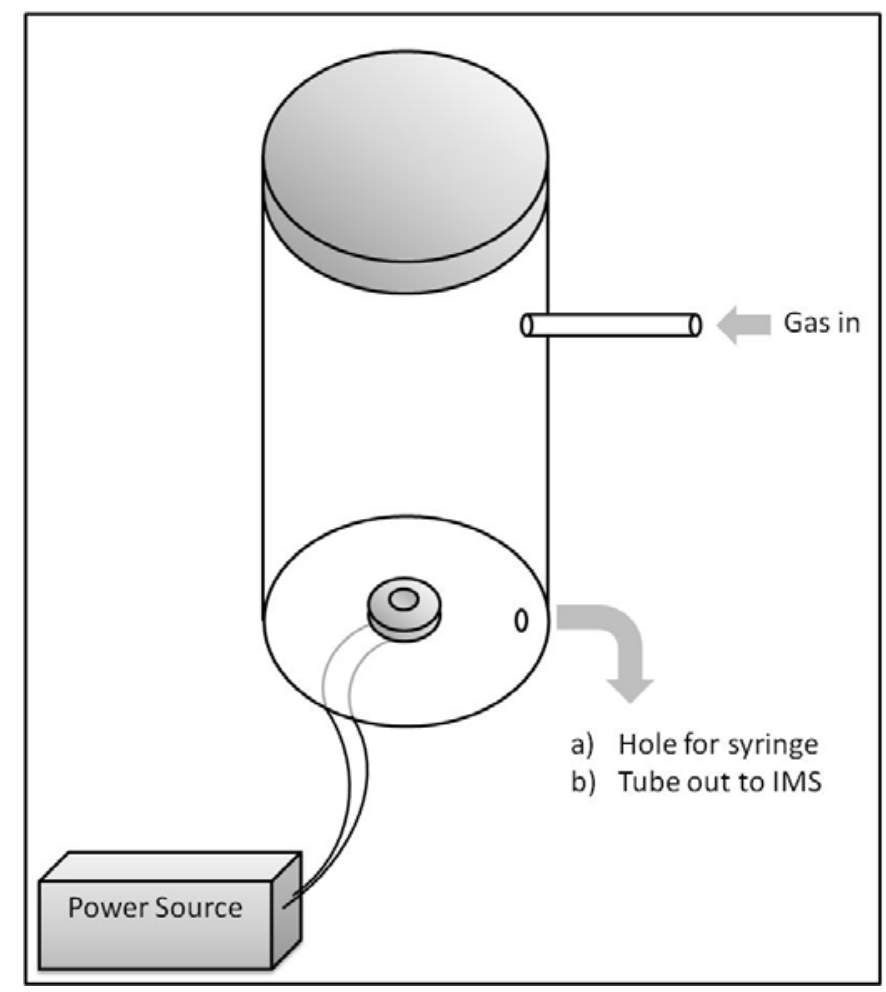

Figure 4.1: A diagram of the Nalgene bottle used for the Static and Dynamic Container experiments. The Static Container was prepared by first by flushing the container with the desired gas, then stopping the flow and withdrawing an aliquot with a syringe. The Dynamic Container used a continuous flow of gas and the outlet was interfaced to the IMS.

The Continuous Flow Tube was configured to allow near real-time control of the ozone and $\mathrm{NO}_{\mathrm{x}}$ concentrations by placing the discharge in a relatively low volume Swagelok cross. A discharge was established in the center of the 1/4" Swagelok stainless steel cross as shown in Figure 4.2. Two 0.026" diameter metal wires housed inside telescoping Teflon tubing (1/16", 1/8" and 1/4") were placed into two opposite openings of the cross. This provided a nearly gas tight seal and allowed for the electrical isolation of the wires. The wires were brought together in the center of the Swagelok cross and separated by $\sim 3 \times 19 \mathrm{~mm}$ section of a $0.018 \mathrm{~mm}$ thick glass microscope slide cover that was held in positioned by the two wires. The power supply used to operate the DPIS was 
connected to these two wires and a discharge occurred at the point where the wires contacted the dielectric. Zero air, at a rate of about $150 \mathrm{~mL} / \mathrm{min}$, was introduced into one of the remaining openings of the cross perpendicular to the wires and flowed parallel to the slide cover, then out the opposing opening. The outlet flow was split with a tee and two metering valves so that part of the effluent (typically a few mL) went to the inlet of the IMS and the remainder was vented.

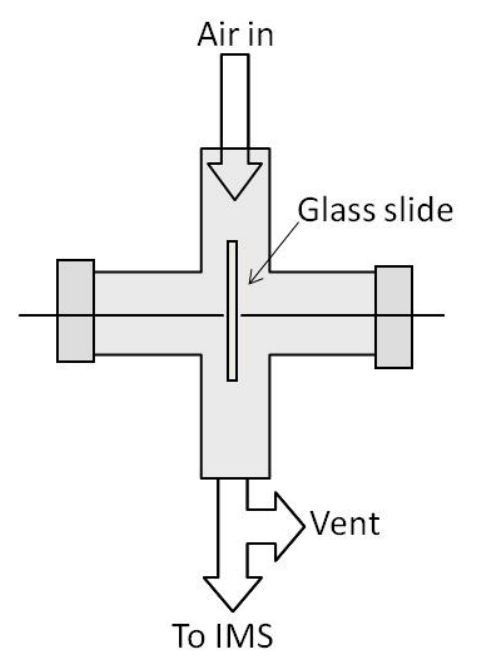

Figure 4.2: A diagram of the Continuous Flow Tube apparatus consisting of a 1/4" stainless steel Swagelok cross. The discharge occurs at the center of this cross where two 0.026" diameter metal wires meet separated by a glass slide. A continuous stream of purified air passes over the discharge and a fraction of the effluent is presented to the inlet of the IMS.

The original IMS inlet consisted of a 1/8" Swagelok fitting used for vapor introduction. In order to introduce a sample of RDX while adding effluent from the discharge source, this inlet was modified to include the addition of a $1 / 8$ " tee so that a wire could be inserted into the heated portion of the IMS inlet. The air flow from the discharge came in via the arm of the tee so the air could flow across the wire and into the 
IMS ionization region carrying both the RDX sample and the discharge effluent. About 1 $\mu \mathrm{L}$ of a solution containing $30 \mathrm{ng} \mathrm{RDX}$ in methanol/acetonitrile was placed on the tip of the wire and the solvent was allowed to evaporate before the wire was inserted into the inlet.

\section{Results and Discussion}

The Static Container was designed to test if neutrals (ozone and $\mathrm{NO}_{\mathrm{x}}$ ), generated in a plasma, could be added to a standard IMS ionization region containing ${ }^{63} \mathrm{Ni}$ and produce the types of ions generated in a corona discharge ionization source. The resulting spectra are shown in Figure 4.3, with Figure 4.3a representing clean IMS-F spectrum of zero air. The DPIS was operated for 1 minute in the flask that had previously been purged for several minutes with purified air. The DPIS was then shut off and a $2 \mathrm{~mL}$ sample of air was extracted with a $3 \mathrm{~mL}$ disposable syringe and injected into the IMS. The resulting spectrum is displayed in Figure 4.3b. The smaller peak on the left was identified as $\mathrm{NO}_{2}^{-}$ and the larger peak as $\mathrm{O}_{2}{ }^{-}$using the IMS-SIM mode of operation (data not shown). Note the slight increase in the $\mathrm{NO}_{2}{ }^{-}$ion and subsequent decrease of the $\mathrm{O}_{2}{ }^{-}$ion with the addition of gas from the discharge source. This indicates that low levels of gasses from the discharge source lead to the production of $\mathrm{NO}_{2}{ }^{-}$ions within the ${ }^{63} \mathrm{Ni}$ ionization source of the IMS indicating that $\mathrm{NO}_{2}$ is generated in the discharge. To determine if this was indeed the case and that the addition of ozone in the presence of air in the ${ }^{63} \mathrm{Ni}$ source would not produce $\mathrm{NO}_{2}{ }^{-}$, the study was repeated in the absence of nitrogen in the discharge region. The flask was purged for several minutes with helium to remove the air and then it was purged with oxygen. The discharge was again operated for 1 minute and a 
$2 \mathrm{~mL}$ sample of gas from the flask was added to the IMS. The resulting spectrum is shown in Figure 4.3c. The intensity of the $\mathrm{NO}_{2}{ }^{-}$peak in Figure 4.3c is less than the $\mathrm{NO}_{2}{ }^{-}$ peak in Figure 4.3a and 4.3b. First it appears that $\mathrm{NO}_{2}$ is not generated with the discharge in a nitrogen free environment since the $\mathrm{NO}_{2}{ }^{-}$peak is even lower than the background $\mathrm{NO}_{2}{ }^{-}$peak. It is possible that ozone may play a role in the ionization region, possibly reacting with $\mathrm{NO}_{2}{ }^{-}$, producing other ions seen as small peaks to the left of $\mathrm{NO}_{2}{ }^{-}$and the right of $\mathrm{O}_{2}^{-}$.

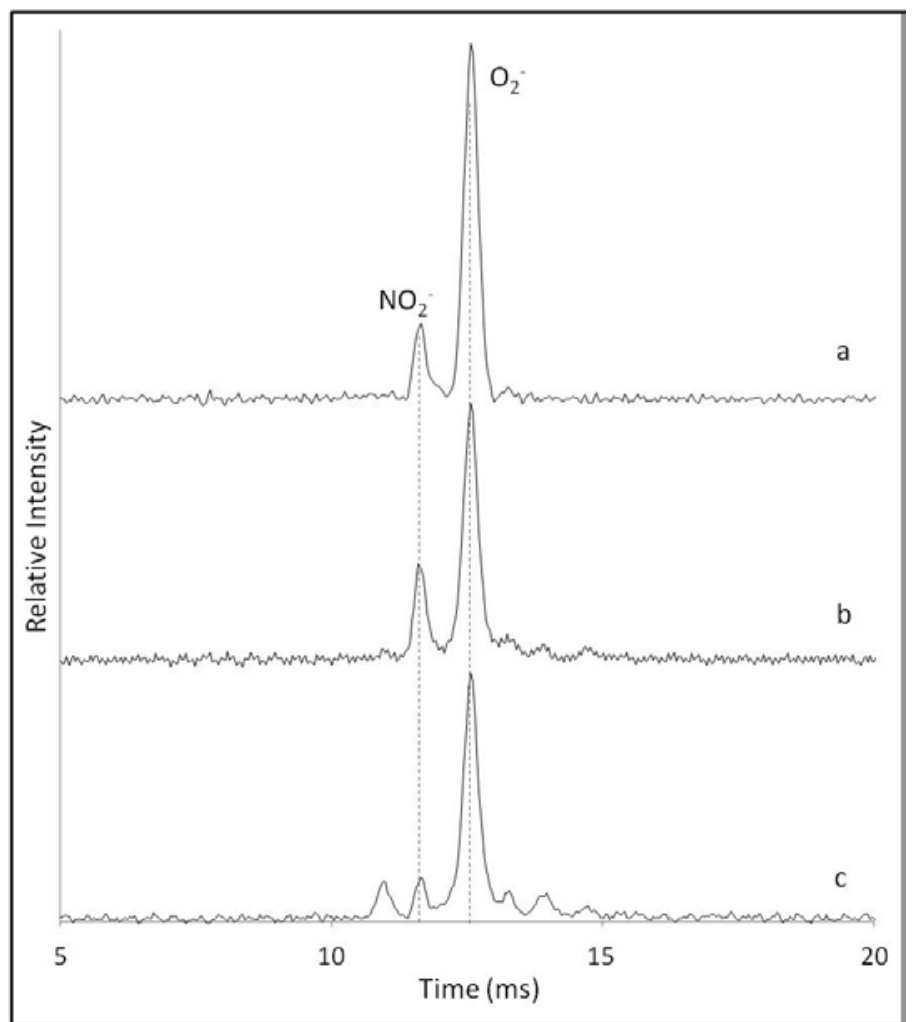

Figure 4.3: IMS-F spectra of (a) zero air, (b) $2 \mathrm{~mL}$ of gas sampled from the Static Container with zero air after 1 minute discharge and (c) $2 \mathrm{~mL}$ of gas sampled from the Static Container with $\mathrm{O}_{2}$ after 1 minute discharge.

Similar to the conditions used to produce Figure 4.3, the Static Container was flushed with purified air and then the DPIS was operated for a longer period of 5 minutes. 
A $2 \mathrm{~mL}$ sample of gas from the Static Container was introduced into the IMS and the resulting spectrum is shown in Figure 4.4a. The ions observed by the MS, as shown in Figure 4.4b, were $\mathrm{O}_{2}{ }^{-}, \mathrm{NO}_{2}{ }^{-}, \mathrm{CO}_{3}{ }^{-}$and $\mathrm{NO}_{3}{ }^{-}$observed at $\mathrm{m} / \mathrm{z}$ values of $32,46,60$ and 62 respectively. The position of these ions is indicated in the mobility spectra in Figure 4.4a and were determined using the mass spectrometer.

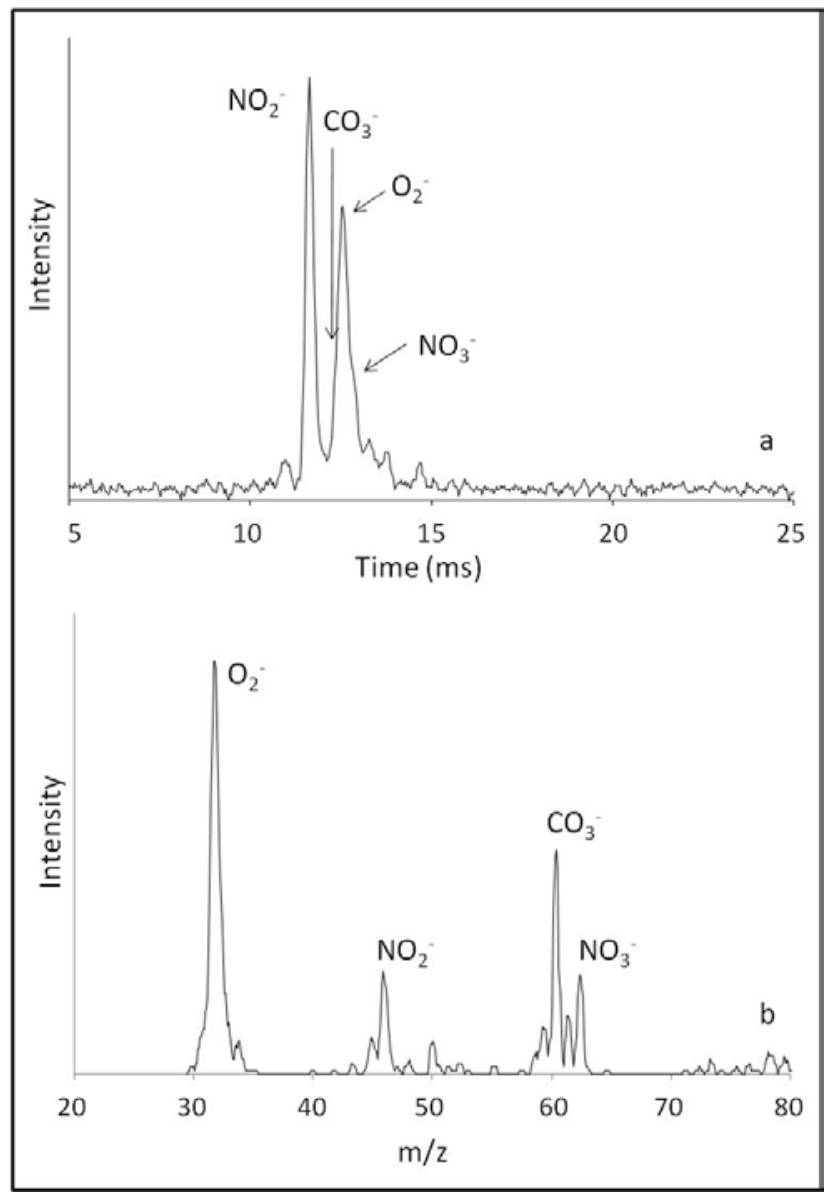

Figure 4.4: (a) ion mobility spectrum and (b) mass spectrum of $2 \mathrm{~mL}$ of gas sampled from the Static Container with zero air after the discharge was operated for 5 minutes.

To link these ions with the respective peaks in the ion mobility spectra, IMS-SIM scans of $\mathrm{m} / \mathrm{z} 32,46,60$ and 62 were acquired and compared to an IMS-EM spectrum. The 
IMS-EM spectrum is shown in Figure 4.5a and the IMS-SIM scans of $\mathrm{m} / \mathrm{z}$ of 46, 60 and 62 are shown in Figure $4.5 \mathrm{c}, \mathrm{d}$ and e, respectively and were collected with $10 \mathrm{~mL} / \mathrm{min}$ of $150 \mathrm{~mL} / \mathrm{min}$ discharge gas added to the ionization source. The IMS-SIM of $\mathrm{m} / \mathrm{z} 32$, shown in Figure 4.5b, is from zero air with no discharge and shows where the $\mathrm{O}_{2}{ }^{-}$ion appears in the mobility spectra as a reference. Reduced mobility values from the IMS-F spectra were calculated to be $2.83,2.65,2.61$ and $2.56 \mathrm{~cm}^{2} \mathrm{~V}^{-1} \mathrm{~s}^{-1}$ for $\mathrm{NO}_{2}{ }^{-}, \mathrm{CO}_{3}{ }^{-}, \mathrm{O}_{2}{ }^{-}$ and $\mathrm{NO}_{3}{ }^{-}$, respectively.

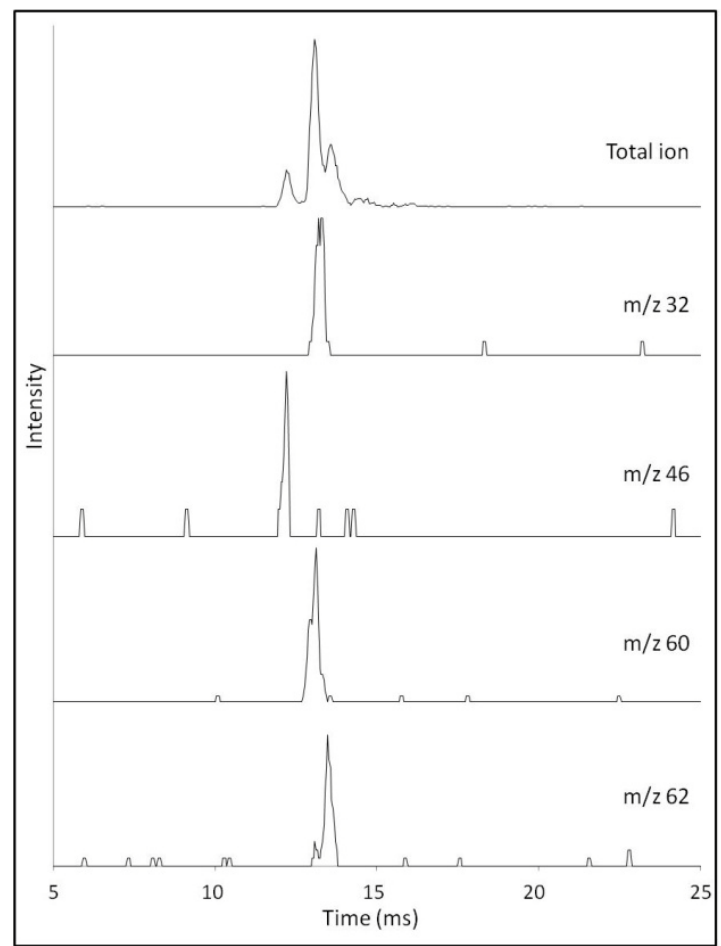

Figure 4.5: IMS spectra of discharge gas from the Continuous Flow Tube collected with the electron multiplier in the mass spectrometer. The IMS-EM, displayed on top, shows the total ion current followed by selected ion monitoring of $\mathrm{m} / \mathrm{z} 32,46,60$ and 62 in order from top to bottom.

In order to monitor the spectra directly as the concentration of ozone and $\mathrm{NO}_{\mathrm{x}}$ increased, the Dynamic Container was used. A continuous flow of $40 \mathrm{~mL} / \mathrm{min}$ passed 
through the bottle and was added to the inlet of the IMS containing a ${ }^{63} \mathrm{Ni}$ ionization source. In Figure 4.6, the x-axis represents the duration of time the discharge source was operated within the bottle. The increase in operation time of the discharge source correlates to an increase in the concentration of ozone and $\mathrm{NO}_{2}$. Figure 4.6 is a plot of the various peak intensities for each specific ion species indicated. The intensities at the locations of each ion species, indicated in Figure 4.4a and identified by mass spectrometry in Figure 4.5, were plotted against the time of discharge operation. The transition from $\mathrm{O}_{2}{ }^{-}$to $\mathrm{NO}_{2}{ }^{-}$to $\mathrm{CO}_{3}{ }^{-}$and finally to $\mathrm{NO}_{3}{ }^{-}$was observed. The $\mathrm{NO}_{3}{ }^{-}$ion became the predominant peak at about 60 seconds and nearly the only peak after about 160 seconds. Plotting the spectra in this manner shows the transition between species that may overlap in the mobility spectra. Specifically $\mathrm{O}_{2}{ }^{-}$and $\mathrm{CO}_{3}{ }^{-}$have essentially the same mobility under these experimental conditions. In Figure 4.6 this shift is observed in the discontinuity seen between the curves for $\mathrm{O}_{2}{ }^{-}$and $\mathrm{CO}_{3}{ }^{-}$. Although this peak appears at the same mobility, further investigation of the mass spectra indicated $\mathrm{O}_{2}^{-}$was dominant early on in the experiment, but it quickly disappeared from the spectra at the onset of the nitrate, nitrite and $\mathrm{CO}_{3}{ }^{-}$ions. 


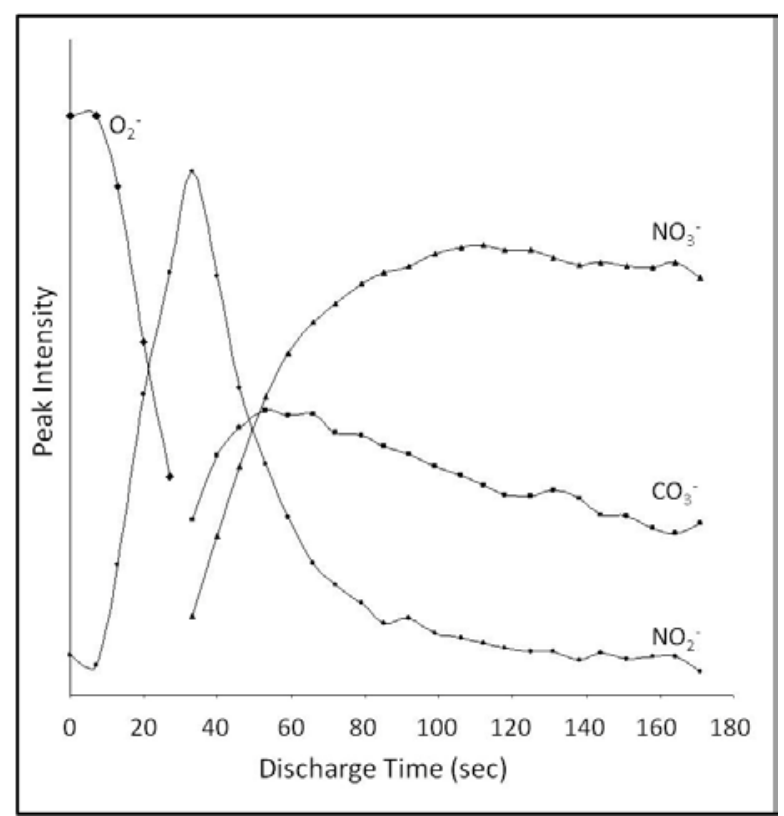

Figure 4.6: IMS peak intensities at increasing discharge times. The source was operated at a $50 \%$ duty cycle with $40 \mathrm{~mL} / \mathrm{min}$ continuous flow of zero air through the Dynamic Container and into the IMS.

The chemical reactions, displayed in Table 4.1, in conjunction with estimated gas concentrations of $\left[\mathrm{N}_{2}\right]=1.4 \times 10^{19} \mathrm{~cm}^{-3},\left[\mathrm{O}_{2}\right]=3.7 \times 10^{18} \mathrm{~cm}^{-3}$ and $\left[\mathrm{CO}_{2}\right]=1.8 \times 10^{12} \mathrm{~cm}^{-3}(0.1$ $\mathrm{ppm}_{\mathrm{v}}$ ) at $140^{\circ} \mathrm{C}$ and 750 torr were used to calculate reaction times. These reaction times along with ion residence times within the IMS are used to explain the relative intensities of ions observed in Figure 4.6.

\begin{tabular}{|l|l|r|}
\hline Chemical Reaction & Rate constant & Reaction \\
\hline $\mathrm{e}^{-}+\mathrm{O}_{2}+\mathrm{N}_{2} \rightarrow \mathrm{O}_{2}^{-}+\mathrm{N}_{2}$ & $\mathrm{k}=1.0 \times 10^{-31} \mathrm{~cm}^{6} / \mathrm{s}$ & $(1)$ \\
\hline $\mathrm{O}_{2}{ }^{-}+\mathrm{NO}_{2} \rightarrow \mathrm{NO}_{2}{ }^{-}+\mathrm{O}_{2}$ & $\mathrm{k}=2.0 \times 10^{-9} \mathrm{~cm}^{3} / \mathrm{s}$ & $(2)$ \\
\hline $\mathrm{O}_{2}{ }^{-}+\mathrm{O}_{3} \rightarrow \mathrm{O}_{3}{ }^{-}+\mathrm{O}_{2}$ & $\mathrm{k}=6.0 \times 10^{-10} \mathrm{~cm}^{3} / \mathrm{s}$ & $(4)$ \\
\hline $\mathrm{O}_{3}{ }^{-}+\mathrm{NO}_{2} \rightarrow \mathrm{NO}_{2}{ }^{-}+\mathrm{O}_{3}$ & $\mathrm{k}=7.0 \times 10^{-10} \mathrm{~cm}^{3} / \mathrm{s}$ & $(5)$ \\
\hline $\mathrm{O}_{3}{ }^{-}+\mathrm{CO}_{2} \rightarrow \mathrm{CO}_{3}{ }^{-}+\mathrm{O}_{2}$ & $\mathrm{k}=5.5 \times 10^{-10} \mathrm{~cm}^{3} / \mathrm{s}$ & $(6)$ \\
\hline $\mathrm{CO}_{3}{ }^{-}+\mathrm{NO}_{2} \rightarrow \mathrm{NO}_{3}{ }^{-}+\mathrm{CO}_{2}$ & $\mathrm{k}=2.0 \times 10^{-10} \mathrm{~cm}^{3} / \mathrm{s}$ & $(7)$ \\
\hline $\mathrm{NO}_{2}{ }^{-}+\mathrm{O}_{3} \rightarrow \mathrm{NO}_{3}{ }^{-}+\mathrm{O}_{2}$ & $\mathrm{k}=1.2 \times 10^{-10} \mathrm{~cm}^{3} / \mathrm{s}$ & \\
\hline
\end{tabular}

Table 4.1: Negative Ion Reaction Rate Constants [46]. 
An example calculation of reaction time is provided for the capture of an electron by oxygen in air from reaction 1 . Other reaction times were calculated in a similar manner using given or estimated neutral concentrations.

$$
\begin{aligned}
& \mathrm{e}^{-}+\mathrm{O}_{2}+\mathrm{N}_{2} \rightarrow \mathrm{O}_{2}^{-}+\mathrm{N}_{2} \quad \mathrm{k}=1.0 \times 10^{-31} \mathrm{~cm}^{6} / \mathrm{s} \\
& \text { Reaction time }=1 /\left(\mathrm{k}^{-}\left[\mathrm{O}_{2}\right]\left[\mathrm{N}_{2}\right]\right) \\
&=1 /\left(1.0 \times 10^{-31} \mathrm{~cm}^{6} / \mathrm{s}\left[3.7 \times 10^{18} \mathrm{~cm}^{-3}\right]\left[1.4 \times 10^{19} \mathrm{~cm}^{-3}\right]\right) \\
&=1.9 \times 10^{-7} \mathrm{~s} \text { or } 190 \mathrm{~ns}
\end{aligned}
$$

The estimate of the concentration for $\mathrm{CO}_{2}$ at $0.1 \mathrm{ppm}_{\mathrm{v}}$ was based upon the manufacture's suggestions of dry air contents $<1 \mathrm{ppm}_{\mathrm{v}}$ as discussed in the experimental section and was assumed to be constant. This concentration was not independently measured and it is recognized that it could be higher due to diffusion of room air into the system through sample introduction. The amount of $\mathrm{NO}_{2}$ and ozone generated was unknown, however it seems reasonable that the amount would continue to increase as the source is operated for longer periods of time. To establish a starting point for calculations, the concentrations of nitrogen dioxide and ozone were set to be equal and three concentrations corresponding to $0.01,0.02$ and $0.1 \mathrm{ppm}_{\mathrm{v}}$ were used to compare subsequent reaction times. The calculated reaction times under these conditions are provided in Table 4.2. 


\begin{tabular}{|c|c|c|c|}
\hline & \multicolumn{3}{|c|}{ Concentrations of $\mathrm{NO}_{2}=\mathrm{O}_{3}$} \\
\hline Reaction & $1.8 \times 10^{11} \mathrm{~cm}^{-3}(0.01 \mathrm{ppm})$ & $3.5 \times 10^{11} \mathrm{~cm}^{-3}(0.02 \mathrm{ppm})$ & $1.8 \times 10^{12} \mathrm{~cm}^{-3}(0.1 \mathrm{ppm})$ \\
\hline 1 & $190 \mathrm{~ns}$ & $190 \mathrm{~ns}$ & $190 \mathrm{~ns}$ \\
\hline 2 & $2.8 \mathrm{~ms}$ & $1.4 \mathrm{~ms}$ & $0.28 \mathrm{~ms}$ \\
\hline 3 & $9.3 \mathrm{~ms}$ & $4.8 \mathrm{~ms}$ & $0.93 \mathrm{~ms}$ \\
\hline 4 & $7.9 \mathrm{~ms}$ & $4.1 \mathrm{~ms}$ & $0.79 \mathrm{~ms}$ \\
\hline 5 & $1.0 \mathrm{~ms}$ & $1.0 \mathrm{~ms} \mathrm{~ms}$ \\
\hline 6 & $28 \mathrm{~ms}$ & $14 \mathrm{~ms}$ & $2.8 \mathrm{~ms}$ \\
\hline 7 & $46 \mathrm{~ms}$ & $24 \mathrm{~ms}$ & $4.6 \mathrm{~ms}$ \\
\hline
\end{tabular}

Table 4.2: Reaction times for reactions 1-7 with varying concentrations of $\left[\mathrm{NO}_{2}\right]=$ $\left[\mathrm{O}_{3}\right]$ as indicated.

With the exception of the electrons, the ions formed here had drift times on the order of between $10-15 \mathrm{~ms}$ in a $10 \mathrm{~cm}$ drift region. The ionization region is $5 \mathrm{~cm}$, thus ion residence times within this region are on the order of about $5 \mathrm{~ms}$. Reaction (1) is always ongoing and fast, occurring in about $190 \mathrm{~ns}$. In air the $\mathrm{O}_{2}{ }^{-}$ion is the major ion observed, often along with a peak at $\mathrm{m} / \mathrm{z} 76$ corresponding to $\mathrm{O}_{2}{ }^{-} \cdot \mathrm{CO}_{2}$. With the introduction of $\mathrm{NO}_{2}$ and $\mathrm{O}_{3}$, the $\mathrm{O}_{2}^{-}$generated by reaction (1) has the opportunity to react to form the $\mathrm{NO}_{2}$ and $\mathrm{O}_{3}$, as shown in reactions (2) and (3). At the selected concentrations of $0.01 \mathrm{ppm}_{\mathrm{v}}$ for the second column in Table 2, the reaction time to form $\mathrm{NO}_{2}{ }^{-}$occurs in less than $3 \mathrm{~ms}$ and should be observed in the IMS spectra. All other reaction times are slow, with the exception of reaction (5), compared to the time scale of the IMS, and reaction (5) depends first upon the production of $\mathrm{O}_{3}{ }^{-}$in reaction 3 and thus should not occur in the experimental time scale. This trend is observed in the first 30 seconds in Figure 4.5. As the concentration of ozone and $\mathrm{NO}_{2}$ increases, $\mathrm{NO}_{2}{ }^{-}$continues to increase and reaches a peak at around 35 seconds. This is also the point where $\mathrm{CO}_{3}{ }^{-}$and $\mathrm{NO}_{3}{ }^{-}$begin to form. The 
intensity of the $\mathrm{NO}_{2}^{-}$peak drops off as $\mathrm{NO}_{2}^{-}$reacts with increasing levels of ozone forming $\mathrm{NO}_{3}{ }^{-}$. The nitrate ion can also be formed with the reaction of $\mathrm{CO}_{3}{ }^{-}$and $\mathrm{NO}_{2}$. In either case the final ion formed is the nitrate ion which dominates the spectra at high concentrations of neutrals generated in the discharge. $\mathrm{O}_{3}{ }^{-}$does not appear in the IMS spectra under any conditions. This results from the reaction times for equations 4 and 5 being faster than the reaction times for the formation of $\mathrm{O}_{3}{ }^{-}$. Once $\mathrm{O}_{3}{ }^{-}$is formed under these conditions it will quickly react with either $\mathrm{CO}_{2}$ or $\mathrm{NO}_{2}$ to form $\mathrm{CO}_{3}{ }^{-}$or $\mathrm{NO}_{2}{ }^{-}$. Even at a conservative estimated concentration of $\mathrm{CO}_{2}\left(0.1 \mathrm{ppm}_{\mathrm{v}}\right)$ the reaction rate with $\mathrm{O}_{3}{ }^{-}$is about $1 \mathrm{~ms}$ and thus $\mathrm{O}_{3}{ }^{-}$should not survive the transit time in the IMS.

Based on the observation that as the concentration of neutrals generated in an electric discharge increase and the ions formed transition from $\mathrm{NO}_{2}{ }^{-}$to $\mathrm{CO}_{3}{ }^{-}$to $\mathrm{NO}_{3}{ }^{-}$, an attempt was made to establish a method to maintain a stable level of a particular ion. For this purpose the Continuous Flow Tube design was created. The small volume, flow rates and split ratio allowed for controlled and continuous introduction of neutral ozone and $\mathrm{NO}_{2}$ into the inlet of the IMS. With the continuous flow tube, three different flow rates were used to establish the desired predominant ion. A split flow of about $10 \mathrm{~mL}$ from the original $150 \mathrm{~mL}$ passing through the source was added to the inlet of the IMS and was considered a "medium" flow rate. Slight adjustments of \pm a few $\mathrm{mL} / \mathrm{min}$ altered the observed ions and for distinction are labeled low, medium and high flow rates. The resulting spectra are shown in Figure 4.7 with (a) no discharge, (b) low flow, (c) medium flow and (d) high flow. With no discharge gases the $\mathrm{O}_{2}{ }^{-}$ion is the predominant and nearly only peak present. At low flows the $\mathrm{NO}_{2}{ }^{-}$ion is essentially the only peak present. At medium flow settings the $\mathrm{NO}_{2}{ }^{-}, \mathrm{CO}_{3}{ }^{-}$and $\mathrm{NO}_{3}{ }^{-}$were all present with $\mathrm{CO}_{3}{ }^{-}$being the 
highest. At high flow rates the observed ions are almost exclusively either $\mathrm{NO}_{3}{ }^{-}$or the nitric acid cluster with $\mathrm{NO}_{3}{ }^{-}$. With this new design, the ions remained relatively stable for several minutes allowing for introduction of samples to investigate ionization chemistry with different reactant ions.

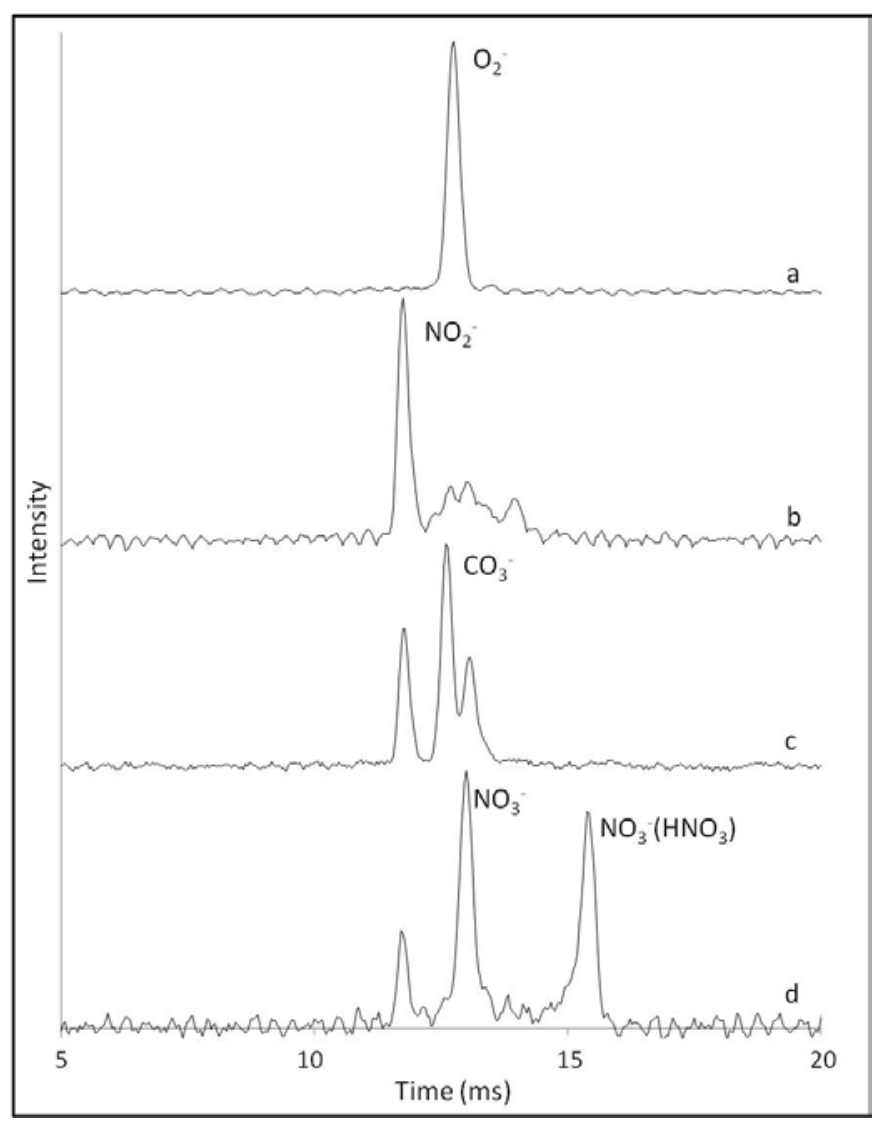

Figure 4.7: IMS-F spectra of discharge gas from the Continuous Flow Tube with (a) no discharge, (b) low flow, (c) medium flow and (d) high flow.

An attempt was made to investigate the changes to the ionization chemistry of an explosive, RDX, which is known to ionize via the formation of adducts with nitrite and chloride anions [19]. Approximately $30 \mathrm{ng}$ of RDX was introduced with each of the three reactant ion chemistries displayed in Figure 4.7 and the results for RDX are shown in Figure 4.8. With nitrite chemistry shown in Figure 4.8a, the $\mathrm{RDX} \cdot \mathrm{NO}_{2}{ }^{-}$adduct is formed 
with a reduced mobility value of $1.49 \mathrm{~cm}^{2} \mathrm{~V}^{-1} \mathrm{~s}^{-1}$. Even when the $\mathrm{CO}_{3}{ }^{-}$ion is the predominant ion, the adduct $\mathrm{RDX} \cdot \mathrm{CO}_{3}{ }^{-}$, is not observed as shown in Figure $4.8 \mathrm{~b}$. This adduct has been seen with a corona discharge mass spectrometer in our laboratory but was not observed in the IMS spectra nor in the mass spectra alone (data not shown). This adduct is believed to have too short of a lifetime to survive the transit time of several milliseconds through the drift tube of the IMS. RDX appears to form a stable adduct with nitrate ions as observed in Figure $4.8 \mathrm{c}$ with a reduced mobility values for $\mathrm{RDX} \cdot \mathrm{NO}_{3}{ }^{-}$of $1.436 \mathrm{~cm}^{2} \mathrm{~V}^{-1} \mathrm{~s}^{-1}$. Even though the nitrate anion appears as two separate peaks, with the predominant ion the nitric acid adduct, the $\mathrm{RDX} \cdot \mathrm{NO}_{3}{ }^{-}$adduct readily forms indicating a stronger interaction with the RDX by displacement of $\mathrm{HNO}_{3}$. $\mathrm{RDX}$ is a good example of how changing the reactant ion species can provide different product ions with unique mobility values. 


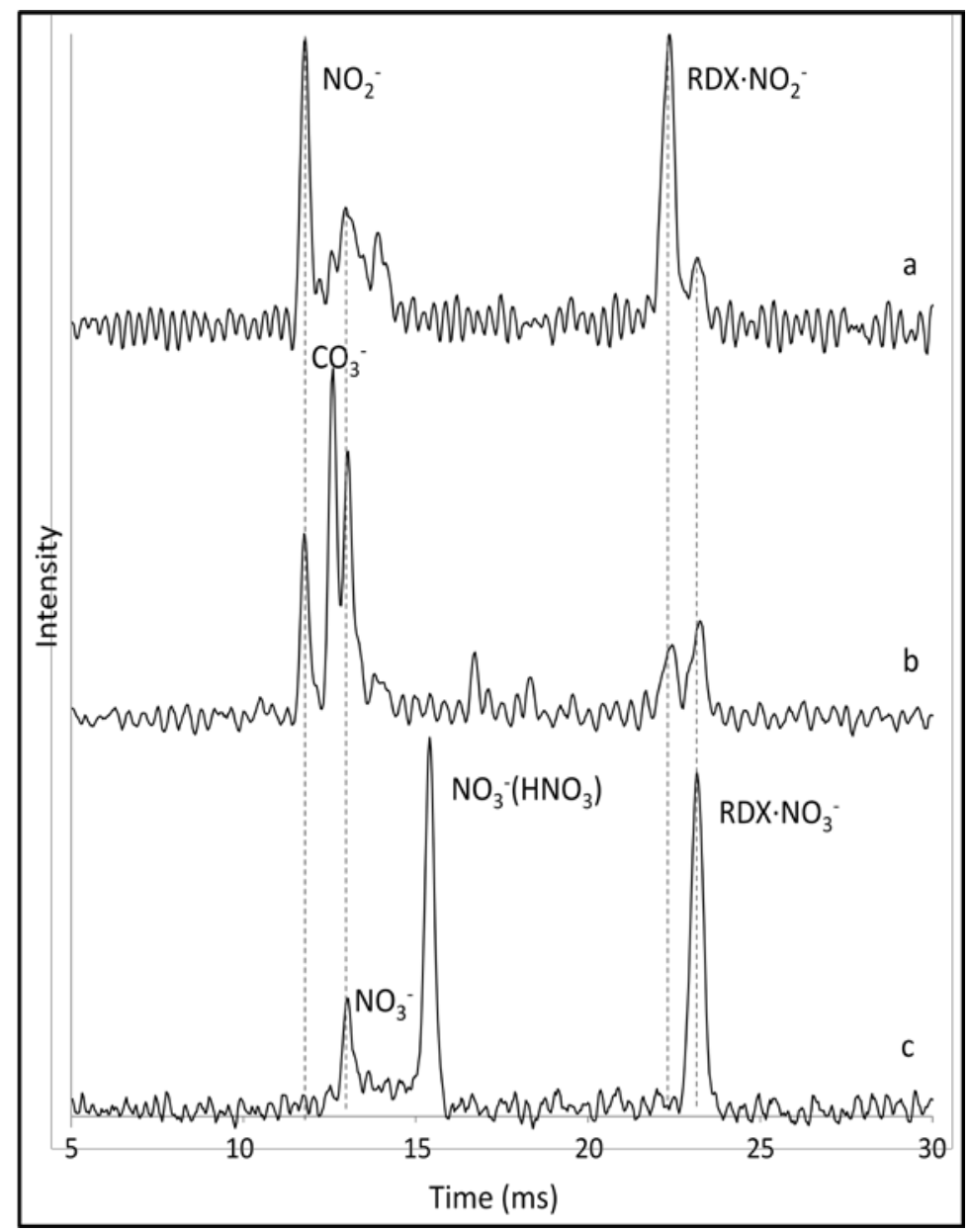

Figure 4.8: IMS-F spectra of RDX with different ion chemistries generated from varying levels of discharge gas from the Continuous Flow Tube (a) low flow, (b) medium flow and (c) high flow. 


\section{CHAPTER 5}

\section{ATMOSPHERIC PRESSURE CHEMICAL IONIZATION OF EXPLOSIVES}

Explosives ionize differently based on their functional groups. For example, nitrotoluenes will ionize via electron attachment or by proton abstraction with $\mathrm{O}_{2}^{-}$or $\mathrm{Cl}^{-}$ [19]. In the presence of $\mathrm{O}_{2}{ }^{-}$, nitroamines or nitrate esters typically ionize to produce nitrite and nitrate ions followed by autoionization producing adducts of the analyte with $\mathrm{NO}_{2}{ }^{-}$or $\mathrm{NO}_{3}{ }^{-}[20]$. Since this is a 2-step process it is less efficient than single step ionization. The addition of chloride reagent ions enhanced the sensitivity for the detection of nitroamine or nitrate ester by producing a ${\mathrm{M} \cdot \mathrm{Cl}^{-}}^{-}$adduct [48]. Some other explosives such as tetryl are not well characterized or discussed in the literature.

The purpose of this research was to investigate the ionization properties of various explosives with a variety of reactant ions. The use of different reactant ions may improve selectivity by either suppressing background contamination or by moving the IMS peak to a different location through the formation of a different adduct. Further, some explosive adducts fragment at elevated temperatures and it is postulated that different adducts may be stable at higher temperatures.

\section{Experimental}

Chemicals

Chemical standards nitroglycerin (NG), PETN, RDX, tetryl, and TNT were obtained from AccuStandard (New Haven, CT) at $100 \mu \mathrm{g} / \mathrm{mL}$ in acetonitrile. Working 
solutions were made by successive dilution of stock solutions with methanol (Fisher Scientific, Pittsburg, PA). Carbon tetrachloride was also from Fisher Scientific.

\section{PCP IMS/MS/MS}

An ion mobility spectrometer interfaced to a triple quadrupole mass spectrometer IMS/MS/MS model MMS 160 (PCP Inc, West Palm Beach, FL) was used in these experiments. This instrument contains a ${ }^{63} \mathrm{Ni}$ ionization source. Operating conditions were as follows: IMS reaction region: 5cm; IMS drift tube length: $10 \mathrm{~cm}$; IMS drift tube temperature: $110^{\circ} \mathrm{C}$; IMS pressure: 750 torr; drift gas: air at $600 \mathrm{ml} / \mathrm{min}$; carrier gas: air at $100 \mathrm{ml} / \mathrm{min}$; electric field: $3000 \mathrm{~V}$ total or $200 \mathrm{~V} / \mathrm{cm}$; gate width: 200 us. The interface region, pumped by a mechanical rough pump, was maintained at a pressure of 280 millitorr. Zero air was produced from compressed house air and passed through a Pure Air Generator model CO2RP140 (Domnick Hunter, Charlotte, NC). Data collection and instrument control were performed using a PC operating with MS Dos version 6.00 and using KEY 3 version 1.21 software (Keystone Applications). Four types of spectra were obtained with this system including: normal mass spectra; ion mobility spectra with the Faraday plate at the end of the drift region (IMS-F); ion mobility spectra with the electron multiplier (IMS-EM); and ion mobility spectra with selected ion monitoring (IMS-SIM). The mass spectra were collected by operating the IMS with the ion gates fully open. The IMS-F spectra were used to measure drift times and subsequent calculation of the reduced mobility values $\left(\mathrm{K}_{\mathrm{o}}\right)$ reported in this paper. IMS-EM spectra, which used the electron multiplier from the mass spectrometer as the detector while the quadrupoles were operated to pass all ions, produced essentially identical spectra compared with IMS- 
F with the exception of a shift of approximately $0.5 \mathrm{~ms}$ to longer drift times. This delay is attributed to the time required for the ions to pass through the interface region and the mass spectrometer, and variances in the shift are due to differences in ion mobility. To identify the drift time of an ion with a specific $m / z$, an IMS-SIM was collected by setting the quadrupoles to pass a specific $\mathrm{m} / \mathrm{z}$ while monitoring the drift time. The drift time of this ion could then be compared to the drift times from the IMS-EM and from this correlated to the IMS-F to link $\mathrm{m} / \mathrm{z}$ to a specific reduced mobility value.

For analyte introduction into the PCP, a dilute solution of the analyte was deposited on a wire and the solvent was allowed to evaporate. The wire was then placed in the IMS so the sample desorbed off of the wire into the instrument. NG, RDX, tetryl, and TNT were run on this instrument. The $\mathrm{O}_{2}{ }^{-}$reactant ion was the standard reactant ion produced with a ${ }^{63} \mathrm{Ni}$ ionization source operated in air. The production of a chloride reactant was accomplished by introducing carbon tetrachloride into the system from a diffusion tube inside of a vessel through which air was flowing into the instrument. Nitrite and nitrate reactant ions were produced by introducing neutral vapors generated from a DPIS contained in a Swagelok stainless steel cross and the levels of $\mathrm{NO}_{2}{ }^{-}$and $\mathrm{NO}_{3}{ }^{-}$were maintained by varying the gas flows from the DPIS into the IMS source. This device was described in detail elsewhere [47]. The $\mathrm{NO}_{2}{ }^{-}$reactant ion was created with the DPIS operated at about $4 \mathrm{kV}$ and $\mathrm{NO}_{3}{ }^{-}$at about $7.5 \mathrm{kV}$. A flow of about $100 \mathrm{~mL} / \mathrm{min}$ from DPIS was added to the IMS and varied slightly to obtain the desired ions. 


\section{WSU IM-TOFMS}

Another instrument used for this study was an IM-TOFMS constructed at Washington State University (WSU, Pullman) and consisted of an ion mobility spectrometer interfaced to a time-of-flight mass spectrometer. The basic design of the instrument can be obtained elsewhere $[49,50]$. IMS operating conditions were as follows: IMS drift tube length: $15 \mathrm{~cm}$; drift tube temperature: $200^{\circ} \mathrm{C}$ for $\mathrm{RDX}, 110^{\circ} \mathrm{C}$ for other explosives; atmospheric pressure: $\sim 690$ torr; drift gas: $\mathrm{N}_{2}$; drift gas flow rate: $\sim 1000 \mathrm{ml} / \mathrm{min}$; voltage on the target screen $10.12 \mathrm{kV}$; voltage on the ion gate $8.82 \mathrm{kV}$. A ${ }^{63} \mathrm{Ni}$ ionization source was used.

The IMS was connected with the TOFMS by a high pressure interface with a $300 \mu \mathrm{m}$ pinhole from the IMS to the interface and a $300 \mu \mathrm{m}$ skimmer from the interface to the TOFMS. The interface was at 1.7 Torr and the MS at $4 \times 10^{-6}$ Torr. Analyte introduction into the IM-TOFMS was accomplished via microtubing and a syringe pump at $2 \mu \mathrm{l} / \mathrm{min}$. NG, PETN, RDX, and tetryl were run on this instrument. Methylene chloride was added to the solutions when a chloride as a reactant ion was desired. For the nitrate reactant ion, the DPIS, located in a dynamic chamber described elsewhere, [47] was used.

\section{API-III MS}

The atmospheric pressure ionization triple quadrupole mass spectrometer used in these studies was an Sciex API-III (Thornhill, Ont., Canada). Data was collected on a Macintosh Quadra 400 using API-Tune software provided with the instrument. Typical interface voltages (IN) were between -300 and -600 VDC. The negative corona discharge current was set at $3 \mu \mathrm{A}$. The other lens voltages were as follows: Pinhole $(\mathrm{OR})=-35, \mathrm{R} 0$ 
$=-30, R 1=-27, R 2=45, R 3=70$. UHP Nitrogen $(99.999 \%$ pure, Matheson Tri-gas, Albuquerque, NM) was used as the auxiliary and curtain gases with flows of $2000 \mathrm{~mL} / \mathrm{min}$ and $400 \mathrm{~mL} / \mathrm{min}$, respectively. Purified air obtained from a Zero Air Generator (Puregas, Broomfield, CO) was used as the nebulizer gas and argon was used as the collision gas for collision induced dissociation (CID) studies. For $\mathrm{CO}_{3}{ }^{-}$studies the instrument was operated with the factory corona discharge ionization source. For $\mathrm{NO}_{3}{ }^{-}$ studies the DPIS was placed on the end of the nebulizer probe as discussed in previous work [26]. Carbon tetrachloride was added to samples for a chloride reactant ion. NG, PETN, RDX, tetryl, and TNT were run on this instrument. Samples were deposited into the nebulizer, the solvent allowed to evaporate, then heater and gas flow turned on to desorb sample and direct it into the MS.

\section{Results and discussion}

The negative reactant ions produced in a ${ }^{63} \mathrm{Ni}$ source in air are mainly $\mathrm{O}_{2}^{-}$. In an electric discharge, such as corona discharge or DPIS, air breaks down to form ozone and $\mathrm{NO}_{\mathrm{x}}$. The reactant ions formed in the presence of ozone and $\mathrm{NO}_{\mathrm{x}}$ are $\mathrm{NO}_{2}{ }^{-}, \mathrm{CO}_{3}{ }^{-}$and $\mathrm{NO}_{3}{ }^{-}$along with some $\mathrm{O}_{2}{ }^{-}$and $\mathrm{O}_{3}{ }^{-}$, and the predominant ion was determined based upon the concentration of ozone and $\mathrm{NO}_{\mathrm{x}}$. It was shown that as the concentration increased the reactant ions shifted from $\mathrm{O}_{2}{ }^{-} \rightarrow \mathrm{NO}_{2}{ }^{-} \rightarrow \mathrm{CO}_{3}{ }^{-} \rightarrow \mathrm{NO}_{3}{ }^{-}$[47]. Figures 5.1 and 5.2 show spectra of negative reactant ions for multiple sources on different instruments. IMS (PCP) spectra of reactant ion peaks of $\mathrm{O}_{2}{ }^{-}, \mathrm{Cl}^{-}, \mathrm{NO}_{2}{ }^{-}$, and $\mathrm{NO}_{3}{ }^{-}$are displayed in Figure 5.1 A, B, $\mathrm{C}$ and $\mathrm{D}$, respectively. The reduced mobility values for $\mathrm{O}_{2}{ }^{-}, \mathrm{Cl}^{-}, \mathrm{NO}_{2}{ }^{-}$, and $\mathrm{NO}_{3}{ }^{-}$at $110^{\circ} \mathrm{C}$ are $2.33,2.73,2.52$, and $2.41 \mathrm{~cm}^{2} \mathrm{~V}^{-1} \mathrm{~s}^{-1}$. There is also a large $\mathrm{HNO}_{3} \cdot \mathrm{NO}_{3}^{-}$peak with a 
reduced mobility of $2.09 \mathrm{~cm}^{2} \mathrm{~V}^{-1} \mathrm{~s}^{-1}$ in Figure 5.1D. This nitrate-nitric acid adduct is observed when the DPIS is operated at high voltages and nitric acid is thought to result from reactions in the plasma with $\mathrm{NO}_{3}{ }^{-}$and trace amounts of water in the air.

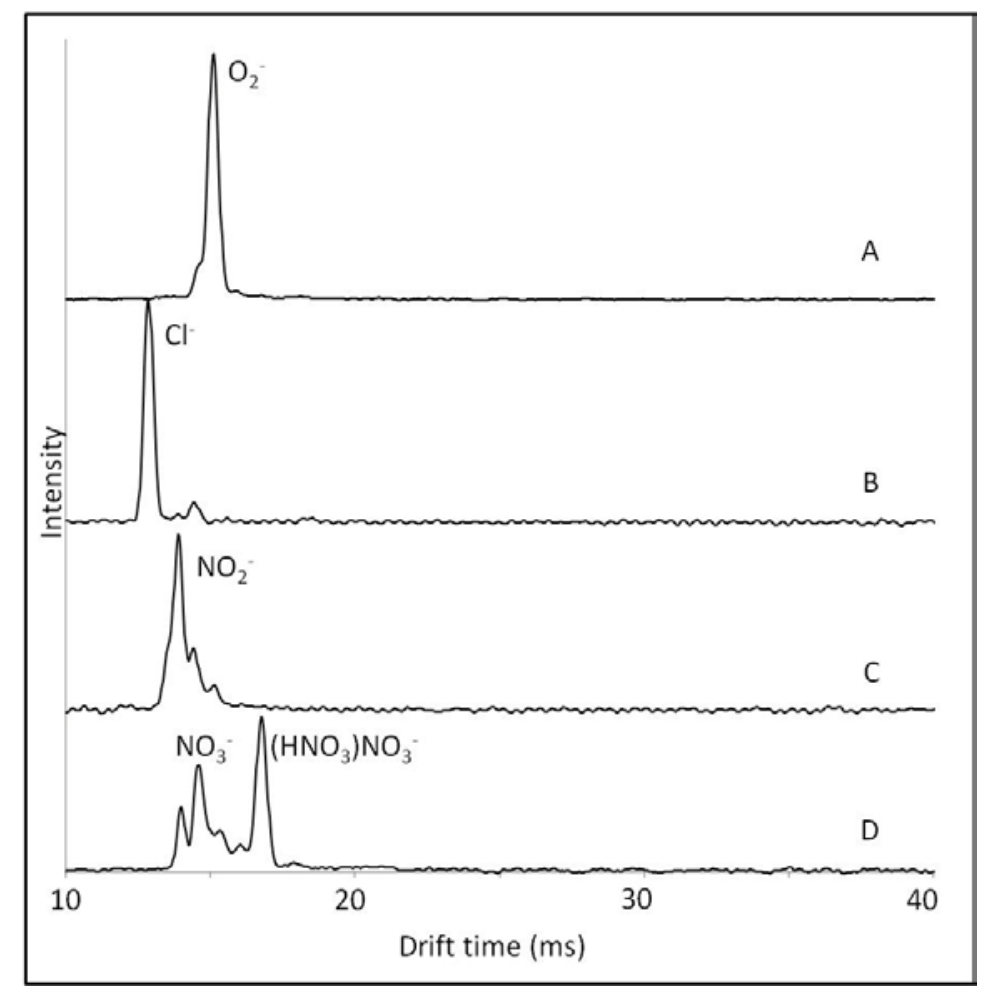

Figure 5.1: IMS Spectra (PCP) of reactant ion peaks generated from a ${ }^{63} \mathrm{Ni}$ ionization source at $110^{\circ} \mathrm{C}$. A) Air showing only $\mathrm{O}_{2}^{-} \mathrm{B}$ ) addition of carbon tetrachloride producing $\mathrm{Cl}^{-}, \mathrm{C}$ ) low concentrations of neutral vapors (ozone and $\mathrm{NO}_{x}$ ) generated in a DPIS producing $\mathrm{NO}_{2}{ }^{-}$and $\mathrm{D}$ ) higher concentrations of neutral vapors (ozone and $\mathrm{NO}_{\mathrm{x}}$ ) generated in a DPIS ionization source producing $\mathrm{NO}_{3}{ }^{-}$and $\mathrm{HNO}_{3} \cdot \mathrm{NO}_{3}{ }^{-}$ions.

Figure 5.2 shows mass spectra (API-III) of reactant ions from a DPIS source with the major ion of $\mathrm{NO}_{3}{ }^{-}$at $\mathrm{m} / \mathrm{z} 62$ (Figure 5.2A), a corona discharge source with the major ion of $\mathrm{CO}_{3}{ }^{-}$at $\mathrm{m} / \mathrm{z} 60$ (Figure 5.2B), and a corona discharge source with carbon tetrachloride added providing the major chloride ion at $\mathrm{m} / \mathrm{z} 35$ and 37 (Figure 5.2C). The ability to specifically produce a given reactant ion by varying ionization parameters such as 
voltage, flow, or through the introduction of a dopant chemical allows for the study of the ionization patterns of explosive molecules with each individual reactant ion.

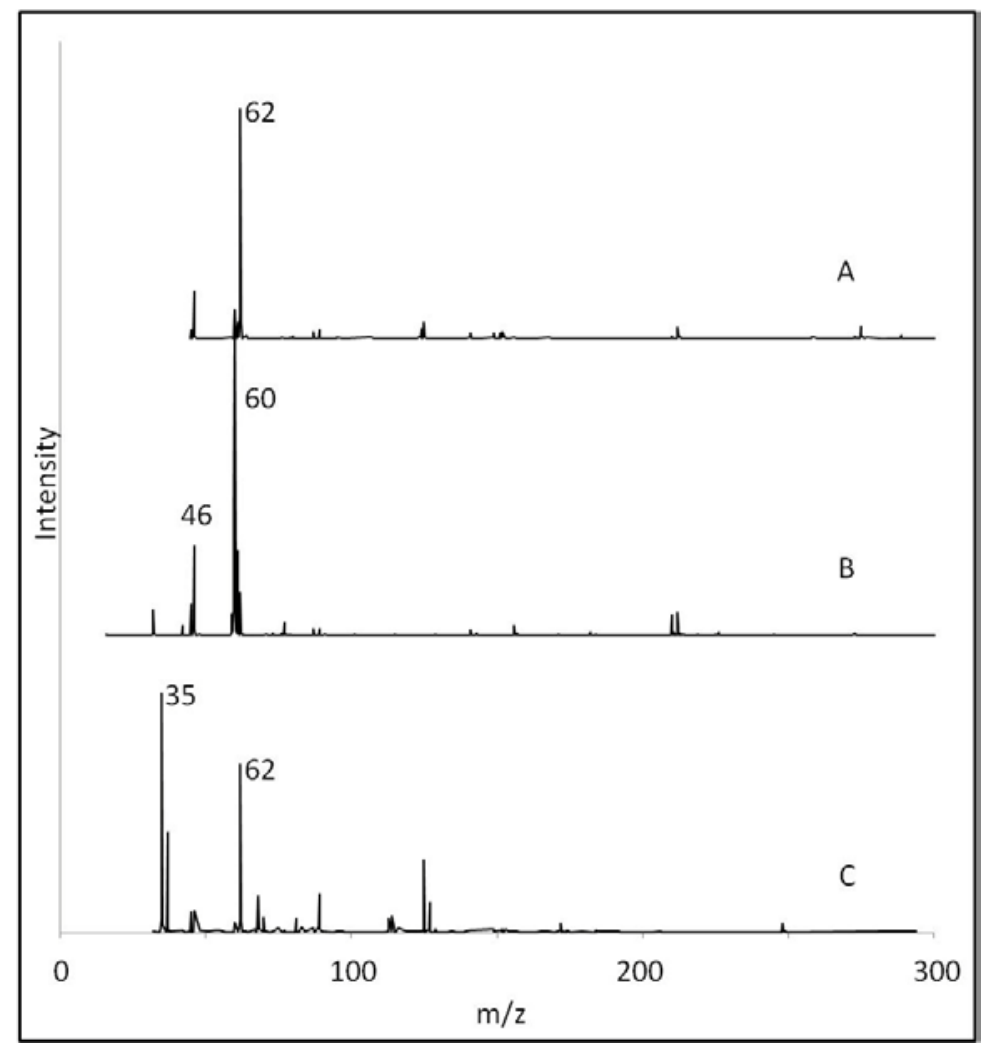

Figure 5.2: Mass spectra (API-III) of clean dry air at ambient temperatures A) using the enclosed DPIS ionization source producing $\mathrm{NO}_{3}{ }^{-}$at $\mathrm{m} / \mathrm{z} 62, \mathrm{~B}$ ) using pointto-plane corona discharge source producing $\mathrm{CO}_{3}{ }^{-}$at $\mathrm{m} / \mathrm{z} 60, \mathrm{C}$ ) using point to plane corona discharge with the addition of carbon tetrachloride producing predominantly $\mathrm{Cl}^{-}$at $\mathrm{m} / \mathrm{z} 35$ and 37 .

The ionization properties of the explosives NG, RDX, TNT, PETN and Tetryl were investigated with reactant ions of $\mathrm{O}_{2}{ }^{-}, \mathrm{Cl}^{-}, \mathrm{NO}_{2}{ }^{-}, \mathrm{CO}_{3}{ }^{-}$and $\mathrm{NO}_{3}{ }^{-}$. The goal was to understand how each explosive compound ionizes and which reactant ion species may provide the optimal ionization characteristics. Another objective was to determine if any of the reactant ion species exhibited selective ionization properties. The ideal selective ionization would be preferential ionization of an explosive compound with little or no ionization of background contamination. Discussed below are the ionization outcomes of 
each individual explosive with a variety of reactant ions. The explosive product ion identities were confirmed by mass spectrometry and the reduced mobility values obtained by IMS results are provided.

Nitroglycerine, when ionized by $\mathrm{O}_{2}{ }^{-}$, produces an $\mathrm{NG} \cdot \mathrm{NO}_{3}{ }^{-}$adduct. This occurs first by the electron transfer from $\mathrm{O}_{2}^{-}$followed by dissociative electron attachment with NG where a nitrate ion is formed. These nitrate ions then further react with other NG molecules to form $\mathrm{NG} \cdot \mathrm{NO}_{3}{ }_{3}$. Since this ionization mechanism is a two-step process it is less efficient than direct ionization. In a point-to-plane corona discharge ionization source $\mathrm{CO}_{3}{ }^{-}$is the major reactant ions formed as part of the ionization of air. Upon the introduction of $\mathrm{NG}$ to the corona discharge, the adduct $\mathrm{NG} \cdot \mathrm{CO}_{3}{ }^{-}$is formed along with $\mathrm{NO}_{3}{ }^{-}$and $\mathrm{NG} \cdot \mathrm{NO}_{3}{ }^{-}$. When a chloride ion is introduced as the reactant ion, the adduct NG. $\mathrm{Cl}^{-}$is observed. When $\mathrm{NO}_{2}{ }^{-}$, produced by the DPIS ionization source, was used as the reactant ion the nitrite adduct was not observed but $\mathrm{NG} \cdot \mathrm{NO}_{3}{ }^{-}$was created. This was thought to occur since the electron affinity of $\mathrm{NO}_{2}{ }^{-}$is much lower than $\mathrm{NO}_{3}{ }^{-}, \mathrm{CO}_{3}{ }^{-}$or $\mathrm{Cl}^{-}$ and the ionization process is then similar to the two-step process observed with $\mathrm{O}_{2}{ }^{-}$ where ionization of $\mathrm{NG}$ produces $\mathrm{NG} \cdot \mathrm{NO}_{3}{ }^{-}$. When $\mathrm{NO}_{3}{ }^{-}$, produced by the DPIS, was used as the reactant ion $\mathrm{NG} \cdot \mathrm{NO}_{3}{ }^{-}$is the only adduct produced.

RDX ionizes similar to the ionization of NG by forming adducts or fragments. The difference with $\mathrm{RDX}$ is that it has $\mathrm{NO}_{2}$ groups instead of $\mathrm{NO}_{3}$ groups that can fragment by dissociative electron attachment to form nitrite ions. These nitrite ions then form adducts with RDX producing the product ions, $\mathrm{RDX} \cdot \mathrm{NO}_{2}^{-}$. This is the main product ion observed for the ionization of $\mathrm{RDX}$ when $\mathrm{O}_{2}{ }^{-}, \mathrm{NO}_{2}{ }^{-}$or $\mathrm{CO}_{3}{ }^{-}$are used as the reactant ions. When chloride is used as the reactant ion, $\mathrm{RDX} \cdot \mathrm{Cl}^{-}$is the only product ion 
observed. When a DPIS with reactant ion $\mathrm{NO}_{3}{ }^{-}$is used, the $\mathrm{RDX} \cdot \mathrm{NO}_{3}{ }^{-}$adduct is observed. The RDX product ions produced with a variety of reactant ions are evident in IMS and MS spectra displayed in Figures 5.3, 5.4, and 5.5.

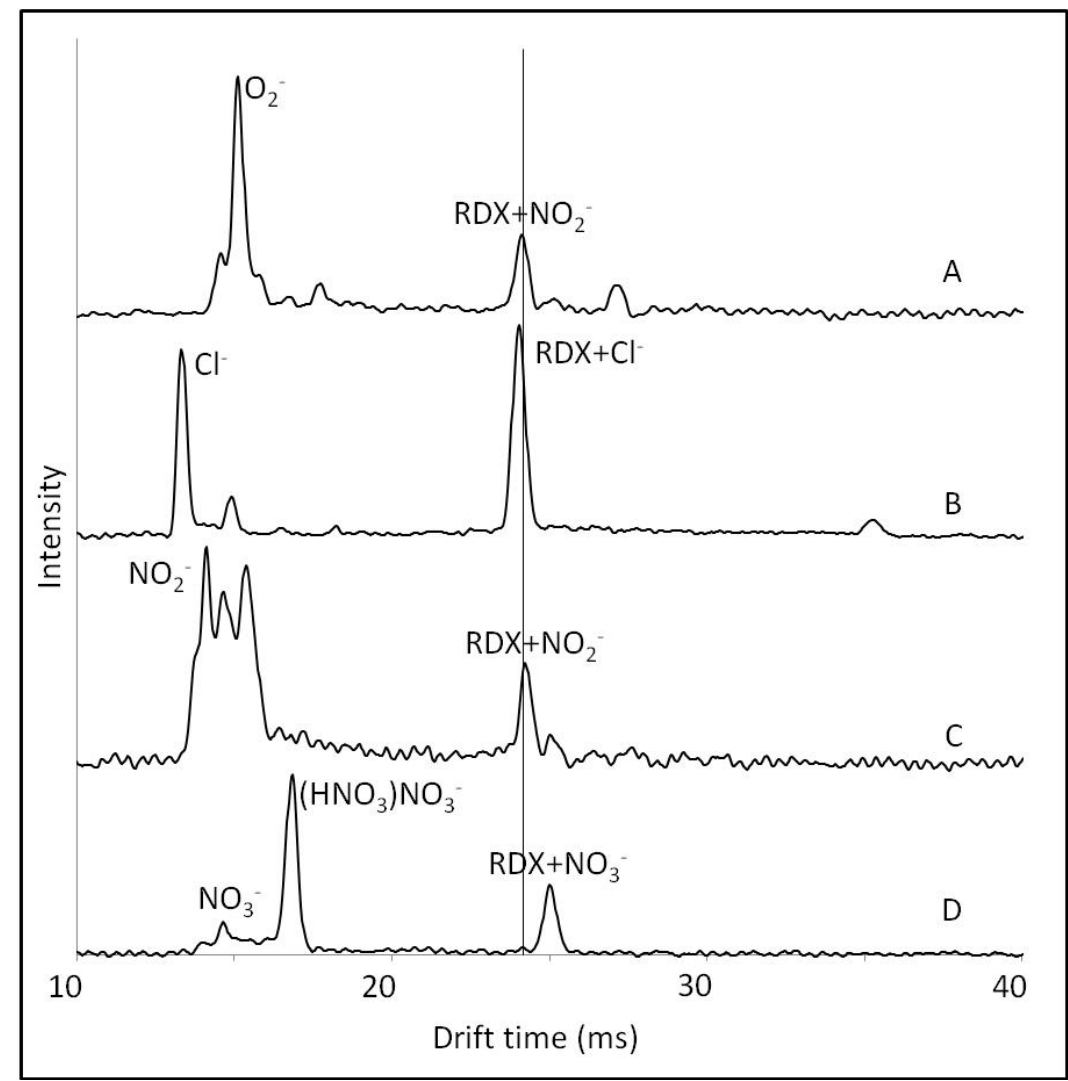

Figure 5.3: IMS spectra (PCP) of $10 \mathrm{ng} \mathrm{RDX}$ with a variety of reactant ion species as shown in Figure 5.1 A) $\left.\left.\mathrm{O}_{2}^{-}, \mathrm{B}\right) \mathrm{Cl}^{-}, \mathrm{C}\right) \mathrm{NO}_{2}{ }^{-}$and D) $\mathrm{NO}_{3}{ }^{-}$and $\mathrm{HNO}_{3} \cdot \mathrm{NO}_{3}{ }^{-}$. 


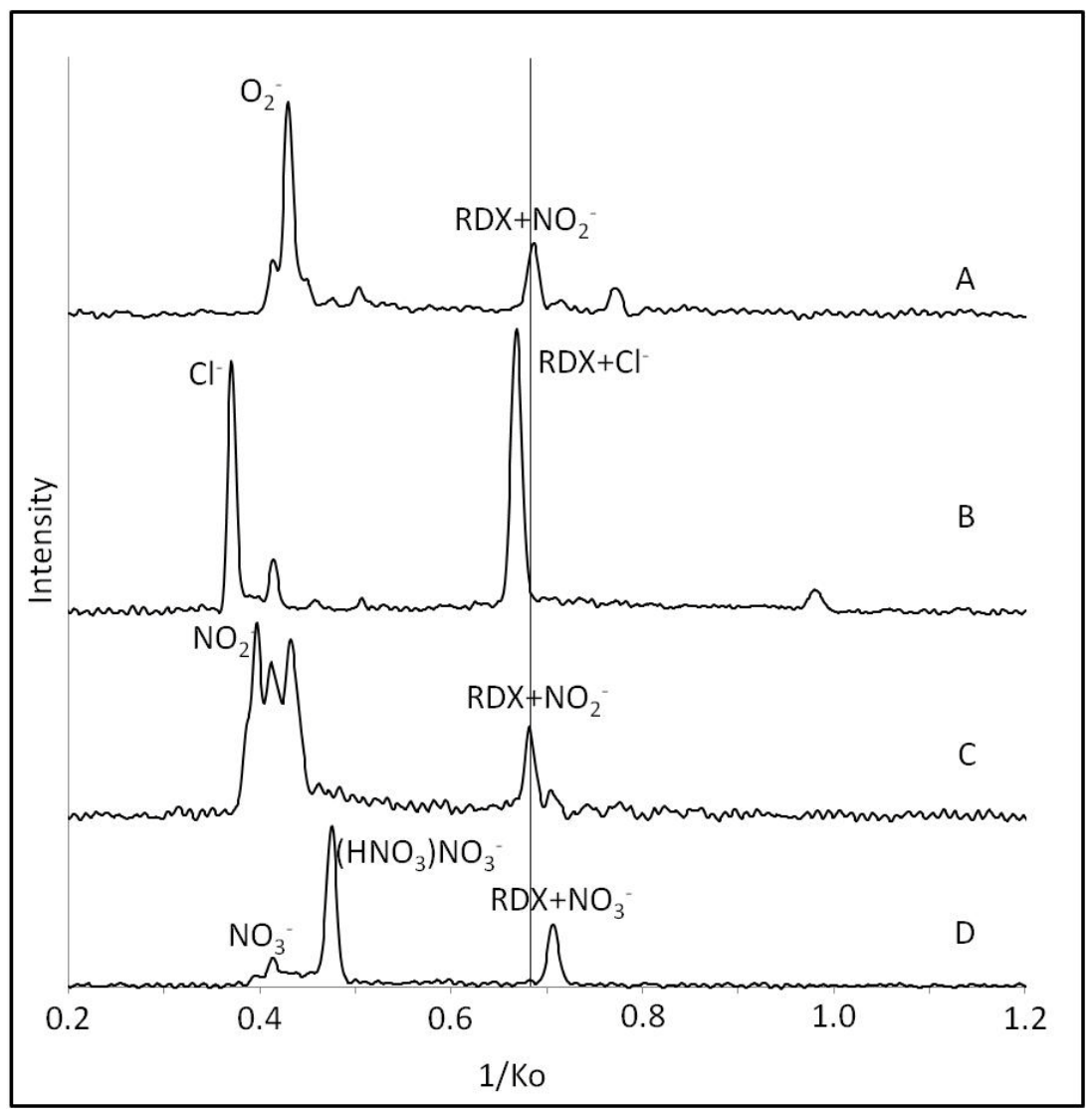

Figure 5.4: IMS spectra with the same data as in Figure 5.3 with the $x$-axis plotted as $1 / K_{0}$.

The IMS spectra of RDX displayed in Figure 5.3 and 5.4 are the same data. The difference between the figures is that in Figure 5.3 the $\mathrm{x}$-axis is provided as drift time in milliseconds whereas in Figure 5.4 it is given as $1 / \mathrm{K}_{\mathrm{o}}$. Although the data were collected at the same temperature, they were collected on different days with small variations in atmospheric pressure. The reduced mobility values are normalized to both temperature and atmospheric pressure. Thus, Figure 5.4 is a more accurate representation of the separation between the various RDX adduct ions. The vertical line displayed in both of these figures is for reference only and centers on the $\mathrm{RDX} \cdot \mathrm{NO}_{2}{ }^{-}$ion. Another interesting feature observable in both the mass spectra and the IMS spectra is when $\mathrm{NO}_{3}{ }^{-}$is used as the reactant ion species. Note the reduction in background peaks other than the $\mathrm{NO}_{3}{ }^{-}$and 
the $\mathrm{RDX} \cdot \mathrm{NO}_{3}{ }^{-}$ions in Figures 5.3D, 5.4D and 5.5A. These spectra appear to be less complex indicating some selectivity in the ionization of RDX with the nitrate reactant ion.

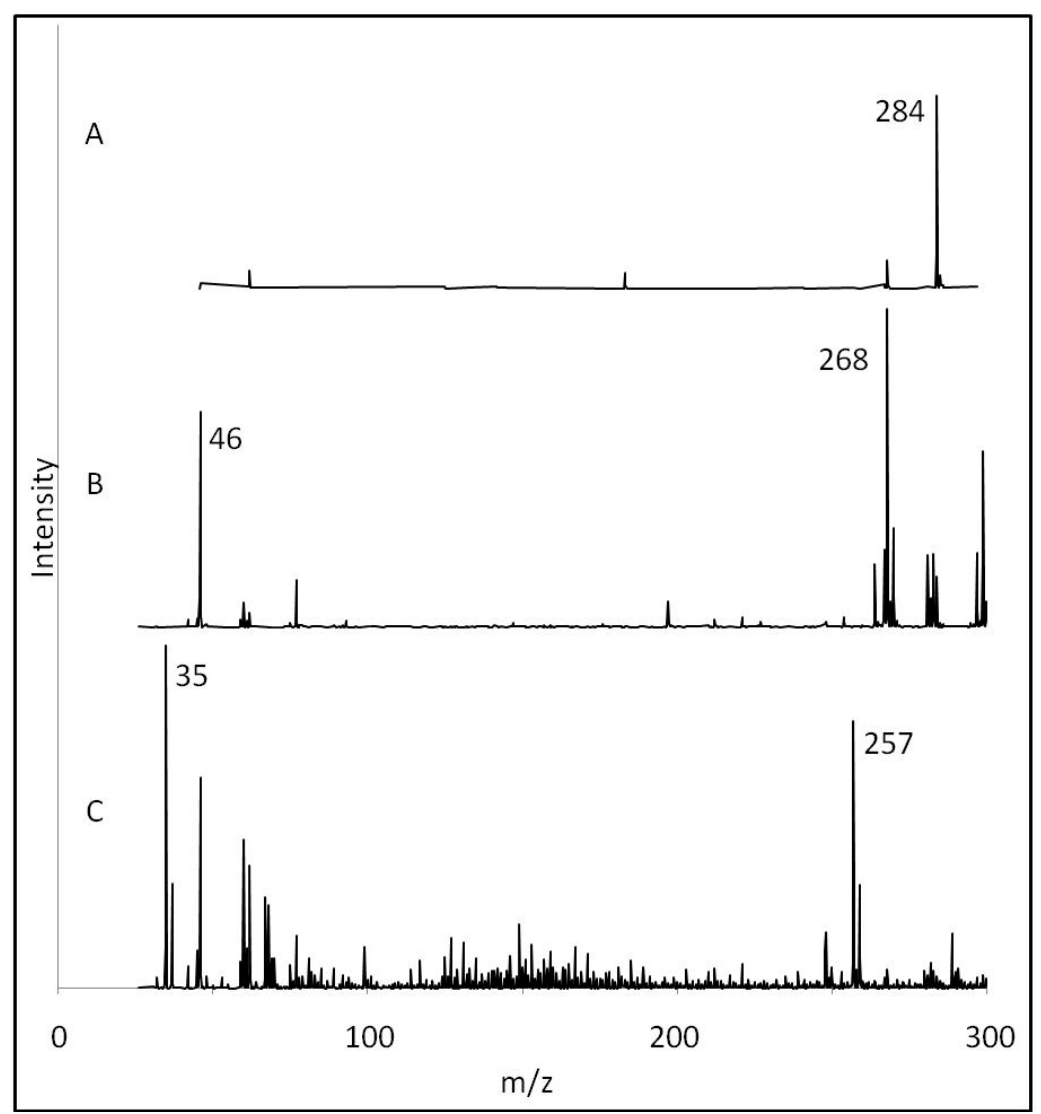

Figure 5.5: Mass spectra (API-III) of RDX at atmospheric pressure with a variety of reactant ion species as shown in Figure 5.2 with $\mathrm{RIPs}$ of $\left.\mathrm{A}) \mathrm{NO}_{3}^{-}, \mathrm{B}\right) \mathrm{CO}_{3}^{-}$, and $\mathrm{C}$ ) $\mathrm{Cl}^{-}$.

Some explosives, like TNT, do not produce adducts. TNT either ionizes by electron attachment or by proton abstraction. The ionization of TNT by $\mathrm{O}_{2}^{-}, \mathrm{Cl}^{-}, \mathrm{NO}_{2}^{-}$, and $\mathrm{CO}_{3}{ }^{-}$produced the proton abstracted species, $(\mathrm{TNT}-\mathrm{H})^{-}$. This is typical of nitro aromatic explosives like TNT where the $\mathrm{NO}_{2}$ groups are attached to a benzene ring, and so are more stable than the $\mathrm{NO}_{2}$ and $\mathrm{NO}_{3}$ groups from $\mathrm{RDX}$ and NG. The hydrogen on 
the methyl group is more acidic than the ring hydrogens and thus is the site for proton abstraction. In the DPIS with $\mathrm{NO}_{2}^{-}$as the reactant ion, (TNT-NO) ${ }^{-}$was also produced, possibly due to a complex and energetic ionization environment containing a variety of reactive species such as ozone. In the corona discharge with $\mathrm{CO}_{3}{ }^{-}, \mathrm{TNT}^{-}$was also produced. This was performed on the API-III mass spectrometer where the ionization occurred right in front of the pinhole and the ion residences times are much shorter than those in an IMS. An interesting event occurred when $\mathrm{NO}_{3}{ }^{-}$ions were used to ionize TNT. The nitrate ion does not appear to ionize TNT, neither by electron transfer nor by proton abstraction. This could be due to the very high electron affinities of the nitrate ion. In the API-III mass spectrometer when the DPIS was used and produced only the nitrate ion, no product ions for TNT were observed. A similar investigation was undertaken with the PCP IMS/MS/MS using the DPIS to generate neutral vapors and introduce these into the ${ }^{63} \mathrm{Ni}$ ionization source to produce the nitrate reactant ion. When $5 \mathrm{ng}$ of TNT was introduced into the IMS only small amounts of (TNT-H)- were observed in the presence of the nitrate reactant ion. The fact that some $(\mathrm{TNT}-\mathrm{H})^{-}$was observed is likely due to the complex ionization occurring within the ionization region. If the TNT reacts with ions, such as $\mathrm{O}_{2}{ }^{-}$prior to them forming $\mathrm{NO}_{3}{ }^{-}$then the proton abstracted TNT could be produced. The reduction of the TNT response in the presence of the nitrate ion was confirmed by adding the same $5 \mathrm{ng}$ of TNT in the presence of chloride ions. When the chloride reactant ions were present, $5 \mathrm{ng}$ of TNT produce relative peak intensities of $634000+/-93000$ compared to those of $224000+/-16000$ for the nitrate reactant ion. These were both done in triplicate. The ionization of TNT here reveals two important conclusions, 1) the nitrate ion can be used as a selective reactant ion and 2) the ionization 
processes occurring the ionization source are complex since the source contains a variety of ions. To take full advantage of the selective ionization process, other methods need to be developed to segregate the analyte from the ionization source so that specific interactions with a single reactant ion can occur.

PETN has the same chemical functionality as NG and ionizes in a similar fashion. PETN when ionized with chloride reactant ions produces PETN.Cl- When nitrate was the reactant ion, $\mathrm{PETN} \cdot \mathrm{NO}_{3}{ }^{-}$was the product ion as can be seen in the 3-D graph from WSU in Figure 5.6. IMS peaks $\mathrm{A}$ and $\mathrm{B}$ are reactant ions $\mathrm{NO}_{3}{ }^{-}$and $\mathrm{NO}_{3}{ }^{-}\left(\mathrm{HNO}_{3}\right)$. Both are shown at $\mathrm{m} / \mathrm{z} 62$ because $\mathrm{NO}_{3}{ }^{-}\left(\mathrm{HNO}_{3}\right)$ fragments easily. IMS peak $\mathrm{D}$ is the adduct which is $m / z 378$ as noted by the intense peak with the asterisk. When run on the API-III MS with corona discharge the only product ion formed is $\mathrm{PETN} \cdot \mathrm{CO}_{3}{ }^{-}$.

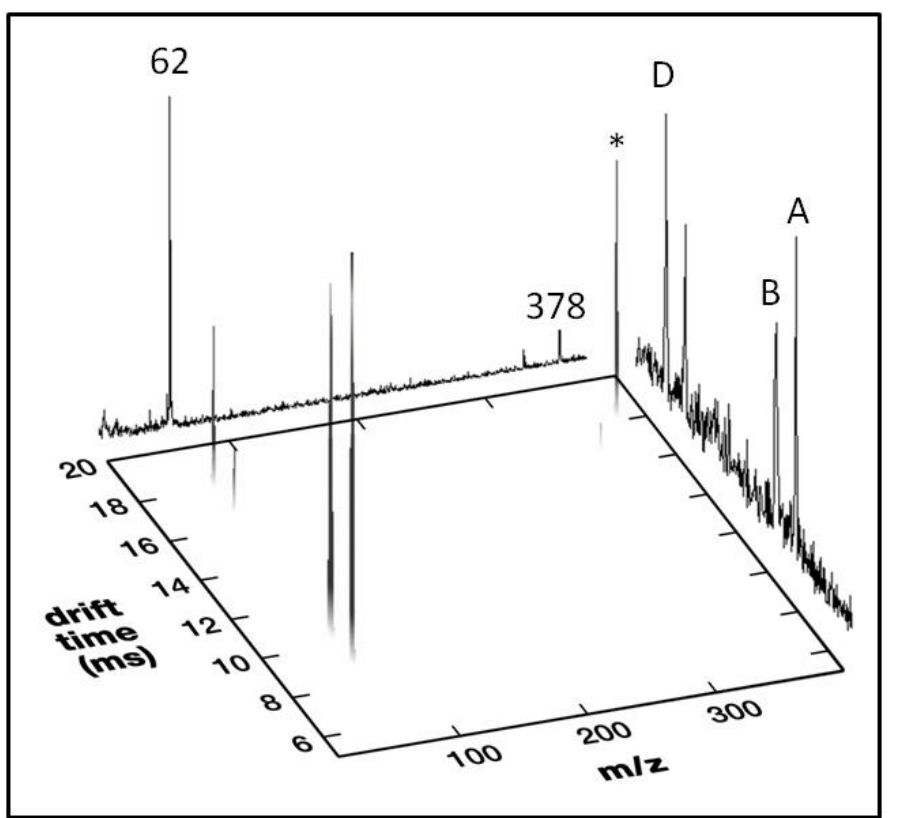

Figure 5.6: Three dimensional IMS and MS spectra (WSU) of PETN with DPIS ionization source at $110^{\circ} \mathrm{C}$ showing $\mathrm{NO}_{3}{ }^{-}$as reactant ion and the $\mathrm{PETN} \cdot \mathrm{NO}_{3}{ }^{-}$ adduct at $\mathrm{m} / \mathrm{z} 378$ and approximately $18.5 \mathrm{~ms}$. 
Tetryl has a similar structure to TNT as it has $\mathrm{NO}_{2}$ groups attached to a benzene ring, so the groups are not expected to fragment. It does however have a $\mathrm{NO}_{2}$ group attached to nitrogen that is connected to the ring. This is the $\mathrm{NO}_{2}$ believed to fragment in order to create the (tetryl- $\left.-\mathrm{NO}_{2}\right)^{-}$ion seen when tetryl is ionized with $\mathrm{O}_{2}{ }^{-}, \mathrm{NO}_{2}{ }^{-}$, and $\mathrm{CO}_{3}{ }^{-}$. Figure 5.7 shows an IMS spectrum of tetryl with $\mathrm{O}_{2}{ }^{-}$as the reactant ion and (tetryl- $\left.\mathrm{NO}_{2}\right)^{-}$ as the product ion. Below the IMS in the figure are an IMS-EM and an IMS-SIM of $m / z$ 241 for mass identification. A small tetryl $\cdot \mathrm{CO}_{3}{ }^{-}$adduct is also seen in the corona discharge. When chloride was the reactant ion tetryl. $\mathrm{Cl}^{-}$was produced. When $\mathrm{NO}_{3}{ }^{-}$was the reactant ion tetryl $\cdot \mathrm{NO}_{3}{ }^{-}$was produced.

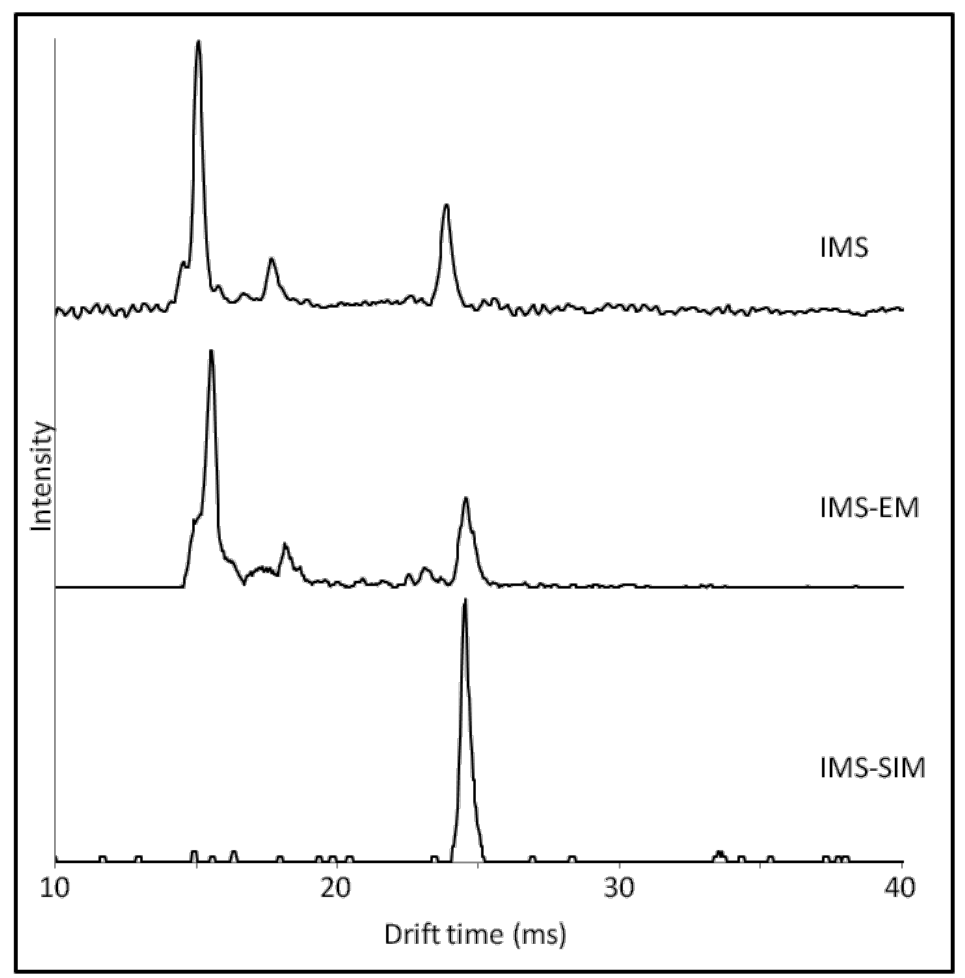

Figure 5.7: IMS/MS spectra (PCP) of tetryl at $110^{\circ} \mathrm{C}$ with $\mathrm{O}_{2}^{-}:$an IMS spectra showing reactant ion peak, an IMS-EM, and an IMS-SIM of $m / z 241$. 


\section{CHAPTER 6}

\section{CONCLUSIONS}

The goals of this research were to form an understanding of the ion formation processes occurring at atmospheric pressure in a discharge source, to investigate the ionization of explosives with various reactant ions, and to find the best reactant ion species available for selective ionization of explosive compounds. Better understanding of the ionization processes will lead to more accurate detection of explosives and the ability to adapt to the detection of new explosive threats.

The major ion in a commercial corona discharge ionization source is $\mathrm{m} / \mathrm{z} 60$, and the identity of it was previously inconclusive. By use of ${ }^{18} \mathrm{O}$ labeled gas and MS/MS, it was determined that the $\mathrm{m} / \mathrm{z} 60$ ion created in a point-to-plane corona discharge is $\mathrm{CO}_{3}{ }^{-}$. Knowing the identity of this major ion aids in the understanding of explosives ionization in a corona discharge. If corona discharge is to be a viable alternative to radioactive ionization sources, the reactant ions created and product ions produced need to be well understood. For example, when NG is ionized in a corona discharge source, a $\mathrm{NG}^{-\mathrm{CO}_{3}}$ adduct is formed. This ion fragments into only $\mathrm{CO}_{3}^{-}$, which shows $\mathrm{CO}_{3}^{-}$has a high electron affinity. If the clusters produced by ionization by $\mathrm{CO}_{3}{ }^{-}$are thermally stable, it could prove a useful tool for the ionization of explosives.

As part of this research a new source, the distributed plasma ionization source (DPIS), was developed and investigated. Like the corona discharge the DPIS is an alternative to radioactive ionization sources. The advantages of the DPIS over point-toplane corona discharge are in the simplicity and ruggedness of its design. The DPIS can 
also be modified to fit nearly any configuration necessary for interfacing to an instrument for use. A lifetime study of the DPIS showed that it could be run constantly for 3 months and still produces a significant amount of ions. The positive ions created in the DPIS were similar to those created with a ${ }^{63} \mathrm{Ni}$ ionization source. The negative ions created were dependent upon the source configuration, voltages and gas flows. In the open configuration ions were similar to the point-to-plane corona discharge ions, with $\mathrm{CO}_{3}{ }^{-}$as the predominant ion. In the enclosed configuration $\mathrm{NO}_{3}{ }^{-}$was the predominant ion. This reactant ion species has not previously been utilized in IMS and could prove a valuable means of ionizing explosives.

While using a DPIS, and varying gas flow, a shift in the reactant ion species from $\mathrm{O}_{2}^{-} \rightarrow \mathrm{NO}_{2}^{-} \rightarrow \mathrm{CO}_{3}{ }^{-} \rightarrow \mathrm{NO}_{3}{ }^{-}$was seen in an IMS. The shift is believed to occur due to increased concentrations of $\mathrm{O}_{3}$ and $\mathrm{NO}_{2}$ in the source region. The DPIS was placed in a container to allow neutrals to build up and bleed into the IMS, and spectra were taken at regular intervals to record the reactant ion intensities. Manipulation of the ionization process became possible when a DPIS was situated inside a Swagelok cross allowing for precise control of gas flow, and thus ozone and $\mathrm{NO}_{\mathrm{x}}$ gas concentrations, delivered into a ${ }^{63} \mathrm{Ni}$ ionization source within an IMS. By controlling gas flows, specific ions were formed. Low flows resulted in a $\mathrm{NO}_{2}{ }^{-}$reactant ion, which changed to $\mathrm{CO}_{3}{ }^{-}$, then to $\mathrm{NO}_{3}{ }^{-}$ at high flows. The ability to choose the reactant ion in an IMS facilitated the study the ionization process of explosives and the ability to probe for the most selective ionization process.

The various reactant ions were used to ionized explosives to determine a variety of product ions and obtain reduced mobilities from IMS. Nitrate and chloride form 
adducts with NG, PETN, RDX, and tetryl. Tetryl fragments to (tetryl- $\left.\mathrm{NO}_{2}\right)^{-}$with both $\mathrm{O}_{2}^{-}$ and $\mathrm{NO}_{2}{ }^{-}$as reactant ions. $\mathrm{RDX}$ forms an adduct with $\mathrm{NO}_{2}{ }^{-}$as a reactant ion, but with an $\mathrm{O}_{2}{ }^{-}$reactant ion $\mathrm{RDX}$ undergoes a multistep reaction in which it fragments $\left(\mathrm{NO}_{2}{ }^{-}\right.$comes off) and creates an $\mathrm{RDX} \cdot \mathrm{NO}_{2}{ }^{-}$adduct. $\mathrm{NG}$ undergoes a similar multistep reaction with $\mathrm{O}_{2}{ }^{-}$ and $\mathrm{NO}_{2}{ }^{-}$as reactant ions, except it forms a NG. $\mathrm{NO}_{3}{ }^{-}$adduct. TNT does not form adducts with any of the reactant ions, but instead ionizes via proton abstraction to give (TNT-H) in most cases. When $\mathrm{NO}_{3}{ }^{-}$is used as the reactant ion with TNT, no ionization occurs. Although this may or may not be a desired outcome, it does provide an example of selective ionization. When nitrate is the predominant ion, the spectrum appears to have less noise than when other reaction ions are present. The reduced mobility values from IMS spectra of all product ions observed are listed in Table 6.1 along with their masses. These values represent mass identified mobility spectra collected under carefully controlled experimental conditions and can be used for comparison purposes to other IMS instruments.

\begin{tabular}{|l|c|c|c|c|c|c|c|c|}
\hline & \multicolumn{2}{|c|}{$\mathrm{O}_{2}^{-}$} & \multicolumn{2}{c|}{$\mathrm{Cl}^{-}$} & \multicolumn{2}{c|}{$\mathrm{NO}_{2}^{-}$} & \multicolumn{2}{c|}{$\mathrm{NO}_{3}^{-}$} \\
\hline Expl (MW) & $\mathrm{K}_{\mathrm{o}}$ & $\mathrm{m} / \mathrm{z}$ & $\mathrm{K}_{\mathrm{o}}$ & $\mathrm{m} / \mathrm{z}$ & $\mathrm{K}_{\mathrm{o}}$ & $\mathrm{m} / \mathrm{z}$ & $\mathrm{K}_{\mathrm{o}}$ & $\mathrm{m} / \mathrm{z}$ \\
\hline NG (227) & 1.37 & 289 & $\begin{array}{c}1.46 \\
{[1.43]}\end{array}$ & 262 & 1.38 & 289 & $\begin{array}{c}1.37 \\
{[1.35]}\end{array}$ & 289 \\
\hline PETN (316) & & & {$[1.23]$} & 351 & & & {$[1.17]$} & 378 \\
\hline RDX (222) & 1.46 & 268 & $\begin{array}{c}1.50 \\
{[1.50]}\end{array}$ & 257 & 1.47 & 268 & $\begin{array}{c}1.42 \\
{[1.42]}\end{array}$ & 284 \\
\hline TNT (227) & 1.56 & 226 & 1.58 & 226 & 1.56 & 226 & & \\
\hline TNT (227) & & & & & 1.62 & 197 & & \\
\hline Tetryl (287) & 1.47 & 241 & $\begin{array}{c}1.33 \\
{[1.31]}\end{array}$ & 322 & 1.48 & 241 & 1.29 & 349 \\
\hline
\end{tabular}

Table 6.1: Reduced mobilities with mass identification of product ion peaks from multiple explosives. Data in brackets is from WSU, all else from PNNL. 


\section{Future work}

The next step in implementation of the DPIS is to build an IMS instrument with the DPIS as its source. Another challenge will be to establish the sample introduction in such a manner that the analyte will interact only with the desired reactant ions and not be ionized with the variety of ions produced in the plasma. Ideally this IMS containing a DPIS will be interfaced to a mass spectrometer to enable mass identification of the ions observed in the IMS.

Being able to interchange the ionization source in the IMS with other ionization

sources, such as ${ }^{63} \mathrm{Ni}$ and point-to-plane corona discharge, would give the ability to find the optimal conditions for each source. Conditions that should be varied include drift tube length, gas flows, and voltage gradient. The length of the drift tube determines the distance the ions have to separate. If the tube is too short the ion peaks will overlap, but if it is too long the peaks will be too wide due to diffusion. The voltage gradient across the drift tube is important because it determines how quickly the ions move through the tube. A wider variety of explosives could also be investigated. Explosives with different functional groups should be studied with multiple sources to understand their ionization process and determine the best instrument parameters for their detection.

The final plan is to develop a multi-tube IMS. In a multi-tube IMS each tube will have a different ionization source, which will cause the chemistry of each tube to be distinct from the others, thus creating different product ions when samples are introduced. The idea behind this arrangement is that if a sample has to go through two or more separate ionization processes, then it forms two or more distinct ions, giving more 
information for the detection algorithm. The more information the alogorithm has, the more accurate the assessment of the sample will be. 


\section{REFERENCES}

[1] Yinon J, Trends in Analytical Chemistry 2002, 21, 292.

[2] Moore DS, Review of Scientific Instruments. 2004, 75, 2499.

[3] Eiceman GA, Stone JA, Analytical Chemistry. 2004, 391A.

[4] Creaser CS, Griffiths JR, Bramwell CJ, Noreen S, Hill CA, Thomas CLP, The Analyst, 2004, 129, 984.

[5] Eiceman GA, Karpas Z. Ion Mobility Spectrometry, 2nd ed. Taylor \& Francis, Boca Raton, 2005.

[6] Poole CF. The Essence of Chromatography. Elsevier, Amsterdam, 2003.

[7] Wu C, Klasmeier J, Hill HH, Rapid Comm. Mass Spectrom., 1999, 13, 1138.

[8] Dzidic I, Carroll DI, Stillwell RN, Horning EC, Analytical Chemistry, 1976, 48, 1763.

[9] Wittmer D, Luckenbill BK, Hill HH, Chen YH, Analytical Chemistry 1994, 66, 2348.

[10] Sielemann S, Baumbach JI, Schmidt H, Pilzecker P, Analytica Chimica Acta, 2001, 431, 293.

[11] Hill, CA, Thomas, CLP, The Analyst, 2003, 128, 55.

[12] Dindosova D, Skalny JD, Acta Phys. Univ. Comenianae, 1992, 33, 77.

[13] Barnes HT, Shaw AN, Zeleny J, Proceedings of the Royal Society of London. Series A, Containing Papers of a Mathematical and Physical Character 1909, 82, 36. 
[14] Taylor SJ, Piper LJ, Conner JA, FitzGerald, Adams JH, Harden CS, Shoff DB, Davis DM, Ewing R,. International Journal for Ion Mobility Spectrometry, 1998, 1,58

[15] Ross SK, Bell AJ, International Journal of Mass Spectrometry, 2002, 218, L1.

[16] Skalny JD, Mikoviny T, Matejcik S, Mason NJ, International Journal of Mass Spectrometry, 2004, 223, 317.

[17] Gravendeel B, de Hoog FJ, J. Phys. B: At. Mol. Phys. 1987, 20, 6337.

[18] http://webbook.nist.gov

[19] Ewing RG, Atkinson DA, Eiceman GA, Ewing GJ, Talanta, 2001, 54, 515.

[20] Khayamian T, Tabrizchi M, Jafari MT Talanta, 2003, 59, 327.

[21] Osborn DL, Leahy DJ, Cyr DR, Neumark DM, J. Chem. Phys. 1996, 13, 5026.

[22] Posey LA, Johnson MA, J. Chem. Phys. 1988, 88, 5383.

[23] Dono A, Paradisi C, Scorrano G, Rapid Comm. Mass Spectrom. 1997, 11, 1687.

[24] Brandvold DK, Martinez P, Atmospheric Environment 1988, 22, 2477.

[25] Siegel MW, Fite WL, J. Phys. Chem. 1976, 80, 2871.

[26] Waltman MJ, Dwivedi P, Hill HH, Blanchard WC, Ewing RG, Talanta 2008, 77, 249.

[27] http://cdiac.ornl.gov/trends/co2/sio-mlo.html

[28] Miller CJ, Ewing RG, 55 ${ }^{\text {th }}$ ACS Northwest Regional Meeting (NORM) “Thermal Stability Study of Nitroglycerin and Structurally Related Chemicals” June 2000. [29] Ewing RG, Blanchard WC, Crawford MJ, Pittsburg Conference, Orlando, FL, March 2005. 
[30] Kaplan K, Hill HH, Gordon Research Conference-Detecting Illicit Substances:

Explosives \& Drugs, Les Diablerets, Switzerland, August 2005.

[31] Na N, Zhang C, Zhao MX, Zhang SC, Yang CD, Fang X, Zhang XR, Journal of Mass Spectrometry 2007, 42, 1079.

[32] Blanchard WC, U.S. patent 7,157,721, 2007.

[33] Wu C, Siems WF, Asbury GR, Hill HH, Analytical Chemistry 1998, 70, 4929.

[34] Asbury GR, Hill HH, Journal of Microcolumn Separations 2000, 12, 172.

[35] LORENTZ, supplied by Integrated Engineering Software, Winnipeg, Manitoba, Canada.

[36] Blanchard WC, Bacon T, Waltman MJ, Ewing RG, $15^{\text {th }}$ International Conference on Ion Mobility Spectrometry, Honolulu, HI, July 2006.

[37] Spangler GE, Carrico JP, International Journal of Mass Spectrometry and Ion Physics 1983, 52, 267.

[38] Spangler GE, Carrico JP, Campbell DN, Journal of Testing and Evaluation 1985, 13,234 .

[39] Wernlund RF, Cohen MJ, Kindel RC, New Concept Symposium and Workshop on Detection and Identification of Explosives, Reston, VA, 1978.

[40] Asbury GR, Klasmeier J, Hill HH, Talanta 2000, 50, 1291.

[41] Huang SD, Kolaitis L, Lubman DM, Applied Spectroscopy 1987, 41, 1371.

[42] Skalny JD, Horvath G, Mason NJ, Journal of Optoelectronics and Advanced Materials 2007, 9, 887.

[43] Hobbs PV. Introduction to Atmospheric Chemistry, Cambridge University Press, Cambridge, 2000. 
[44] Brandvold DK, Martinez P, Dogruel D, Atmospheric Environment 1989, 23, 1881.

[45] Ewing RG, Eiceman GA, Harden, CS, Stone, JA. International Journal of Mass Spectrometry, 2006, 255-256, 76.

[46] Bowers MT ed. Gas Phase Ion Chemistry Vol. 1, Academic Press, New York, 1979.

[47] Ewing RG, Waltman MJ, International Journal for Ion Mobility Spectrometry 2009, 12,65 .

[48] Lawrence AH, Neudorfl P, Analytical Chemistry 1988, 60, 104.

[49] Steiner, WE, Clowers BH, et. al. Rapid Comm. Mass Spectrom. 2001, 15, 2221.

[50] Crawford CL, Boudries H, et. al. Analytical Chemistry, 2010, 82, 387. 